\title{
Recurrence in Linear dynamics
}
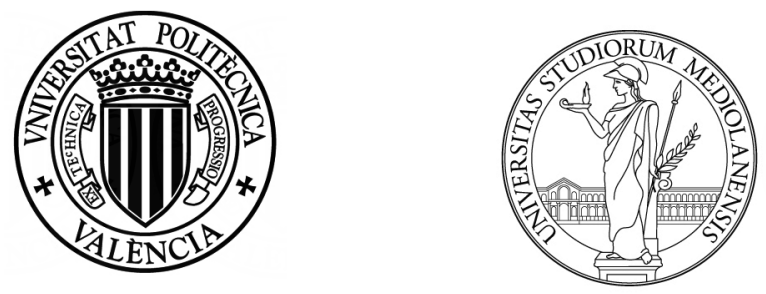

Yunied Puig de Dios

Supervised by

Alfred Peris

Universidad Politécnica de Valencia

Università degli Studi di Milano

A Thesis submitted for the degree of

PhD in Mathematics

July 2014 


\begin{abstract}
A bounded and linear operator is said to be hypercyclic if there exists a vector such that its orbit under the action of the operator is dense. The first example of a hypercyclic operator on a Banach space was given in 1969 by Rolewicz (32) who showed that if $B$ is the unweighted unilateral backward shift on $l^{2}$, then $\lambda B$ is hypercyclic if and only if $|\lambda|>1$. Among its features, we can mention for example that finite-dimensional spaces cannot support hypercyclic operators, proved by Kitai (29). On the other hand, several people have shown in different contexts, in the Hilbert space frame, that the set of hypercyclic vectors for a hypercyclic operator is a $G_{\delta}$ dense set (20), (22), (29).

This thesis is divided into four chapters. In the first one, we give some preliminaries by mentioning some definitions and known results that will be of great help later.

In chapter 2, we introduce a refinement of the notion of hypercyclicity, relative to the set $N(U, V)=\left\{n \in \mathbb{N}: T^{-n} U \cap V \neq\right.$ $\emptyset$ \} when belonging to a certain collection $\mathscr{F}$ of subsets of $\mathbb{N}$, namely a bounded and linear operator $T$ is called $\mathscr{F}$-operator if $N(U, V) \in \mathscr{F}$, for any pair of non-empty open sets $U, V$ in
\end{abstract}


$X$. First, we do an analysis of the hierarchy established between $\mathscr{F}$-operators, whenever $\mathscr{F}$ covers those families mostly studied in Ramsey theory. Second, we investigate which kind of properties of density can the sets $N(x, U)=\left\{n \in \mathbb{N}: T^{n} x \in U\right\}$ and $N(U, V)$ have for a given hypercyclic operator, and classify the hypercyclic operators accordingly to these properties.

In chapter three, we introduce the following notion: an operator $T$ on $X$ satisfies property $\mathcal{P}_{\mathscr{F}}$ if for any $U$ non-empty open set in $X$, there exists $x \in X$ such that $N(x, U) \in \mathscr{F}$. Let $\overline{\mathcal{B D}}$ the collection of sets in $\mathbb{N}$ with positive upper Banach density. We generalize the main result of (19) using a strong result of Bergelson and Mccutcheon (10) in the vein of Szemerédi's theorem, leading us to a characterization of those operators satisfying property $\mathcal{P}_{\overline{\mathcal{B D}}}$. It turns out that operators having property $\mathcal{P}_{\overline{\mathcal{B D}}}$ satisfy a kind of recurrence described in terms of essential idempotents of $\beta \mathbb{N}$ (the Stone-Čech compactification of $\mathbb{N}$ ). We will discuss the case of weighted backward shifts satisfying property $\mathcal{P}_{\overline{\mathcal{B D}}}$. On the other hand, as a consequence we obtain a characterization of reiteratively hypercyclic operators, i.e. operators for which there exists $x \in X$ such that for any $U$ non-empty open set in $X$, the set $N(x, U) \in \overline{\mathcal{B D}}$.

The fourth chapter focuses on a refinement of the notion of disjoint hypercyclicity. We extend a result of Bès, Martin, Peris and Shkarin by stating: $B_{w}$ is $\mathscr{F}$-weighted backward shift if and only if $\left(B_{w}, \ldots, B_{w}^{r}\right)$ is $d-\mathscr{F}$, for any $r \in \mathbb{N}$, where $\mathscr{F}$ runs 
along some filters strictly containing the family of cofinite sets, which are frequently used in Ramsey theory. On the other hand, we point out that this phenomenon does not occur beyond the weighted shift frame by showing a mixing linear operator $T$ on a Hilbert space such that the tuple $\left(T, T^{2}\right)$ is not $d$-syndetic. We also, investigate the relationship between reiteratively hypercyclic operators and $d$ - $\mathscr{F}$ tuples, for filters $\mathscr{F}$ contained in the family of syndetic sets. Finally, we examine conditions to impose in order to get reiterative hypercyclicity from syndeticity in the weighted shift frame. 


\section{Resumen}

Un operador lineal y acotado se dice hipercíclico si existe un vector cuya órbita es densa. El primer ejemplo de operador hipercíclico sobre un espacio de Banach fue dado por Rolewicz en 1969 , quien prueba que $B$ es hipercíclico si y sólo si $|\lambda|>1$, para $B$ operador desplazamiento unilateral en $l_{2}$. Entre los primeros resultados vinculados a la hiperciclicidad podríamos mencionar el hecho que ningún espacio finito dimensional puede soportar un operador hipercíclico y que en el contexto de los espacios de Hilbert, todo operador hipercíclico tiene un conjunto $G_{\delta}$-denso de vectores hipercíclicos.

La tesis está dividida en cuatro capítulos. En el primero, se dan algunos preliminares, repasando aquellas definiciones y resultados ya existentes en la literatura que nos serán necesarios más adelante.

En el capítulo dos, introducimos un refinamiento del concepto de hiperciclicidad, relativo al conjunto $N(U, V)=\{n \in \mathbb{N}$ : $\left.T^{-n} U \cap V \neq \emptyset\right\}$, cuando éste pertenece a una cierta colección de subconjuntos de $\mathbb{N}$. En otras pala-

bras, un operador lineal y continuo $T$ se dice $F$-operador si 
$N(U, V) \in \mathscr{F}$ para cada par de conjuntos abiertos no vacíos $U, V$ de $X$. En primer lugar, hacemos un análisis de la jerarquía establecida entre $\mathscr{F}$-operadores cuando $\mathscr{F}$ recorre aquellas familias más estudiadas en Teoría de Ramsey. En segundo lugar, analizamos qué tipo de propiedades de densidad pueden tener los conjuntos de la forma $N(x, U)=\left\{n \in \mathbb{N}: T^{n} x \in U\right\}$ y $N(U, V)$ para un operador hipercíclico dado. De igual modo, clasificamos los operadores hipercíclicos de acuerdo a estas propiedades.

En el capítulo tres, se introduce la siguiente noción: un operador $T$ en $X$ satisface la propiedad $\mathcal{P}_{\mathscr{F}}$ si para todo conjunto abierto no vacío $U$ de $X$, existe $x \in X$ tal que $N(x, U) \in \mathscr{F}$. Sea $\overline{\mathcal{B D}}$ la familia de los conjuntos de $\mathbb{N}$ con densidad de Banach superior positiva. En primer lugar, generalizamos un resultado de Costakis y Parissis haciendo uso de una versión generalizada del Teorema de Szemerédi, debido a Bergelson y McCutcheon. Como consecuencia obtenemos una caracterización de aquellos operadores que satisfacen la propiedad $\mathcal{P}_{\overline{\mathcal{B D}}}$. Resulta que los operadores teniendo la propiedad $\mathcal{P}_{\overline{\mathcal{B D}}}$ satisfacen un tipo de recurrencia que puede ser descrito en términos de los idempotentes esenciales de $\mathbb{N}$. Se discute también, el caso de los operadores desplazamiento ponderados que satisfacen la propiedad $\mathcal{P}_{\overline{\mathcal{B D}}}$. Por otra parte, se obtiene como consecuencia una caracterización de los operadores reiterativamente hipercíclicos, i.e. operadores para los cuales existe $x \in X$ tal que para todo conjunto abierto no vacío $U$ de $X$, el conjunto $N(x, U) \in \overline{\mathcal{B D}}$. 
En el cuarto capítulo nos enfocamos en el estudio de un refinamiento de la noción de hiperciclicidad disjunta. Por una parte, extendemos un resultado de Bes, Martin, Peris y Shkarin donde afirmamos lo siguiente: $B_{w}$ es $F$-operador si y sólo si $\left(B_{w}, \ldots, B_{w}^{r}\right)$ es $d-F$, para todo $r \in \mathbb{N}$, donde $B_{w}$ denota un operador desplazamiento ponderado en $c_{0}$ o $l_{p},(1 \leq p<1)$ y donde $\mathscr{F}$ es cualquiera de los filtros más usados en Teoría de Ramsey que contienen estrictamente la familia de los conjuntos cofinitos. Por otra parte, se destaca que este fenómeno no tiene lugar fuera del contexto de los operadores desplazamiento ponderados. Para ello se muestra un operador lineal mezclante $T$ en un espacio de Hilbert tal que $\left(T, T^{2}\right)$ no es d-sindético. También se indaga sobre la relación entre operadores reiterativamente hipercíclicos y $d-F$ tuplas, para filtros $\mathscr{F}$ contenidos en la familia de los conjuntos sindéticos. Finalmente, examinamos qué condiciones son necesarias para que un operador desplazamiento ponderado sindético sea reiterativamente hipercíclico. 


\section{Resum}

Un operador lineal i tancat es diu hipercíclic si hi ha un vector l'òrbita del qual és densa. El primer exemple d'operador hipercíclic sobre un espai de Banach va ser donat per Rolewicz en 1969, qui prova que B és hipercíclic si i només si $|\lambda|>1$, per a $\mathrm{B}$ operador desplaçament unilateral en $l_{2}$. Entre els primers resultats vinculats a l'hiperciclicidad podríem mencionar el fet que cap espai finit dimensional no pot suportar un operador hipercíclic i que en el context dels espais de Hilbert, tot operador hipercíclic té un conjunt $G_{\delta}$-denso de vectors hipercíclics.

La tesi està dividida en quatre capítols. En el primer, es donen alguns preliminars, repassant aquelles definicions i resultats ja existents en la literatura que ens seran necessaris més avant.

En el capítol dos, introduim un refinament del concepte de hiperciclicidad, relatiu al conjunt $N(U, V)=\left\{n \in \mathbb{N}: T^{-n} U \cap V \neq\right.$ $\emptyset\}$, quan este pertany a una certa collecció $\mathscr{F}$ de subconjunts de $\mathbb{N}$. En altres paraules, un operador lineal i continu $T$ es diu $\mathscr{F}$-operador si $N(U, V) \in \mathscr{F}$, per a cada parell de conjunts oberts no buits $U, V$ de $X$. En primer lloc, fem una anàlisi de la jerarquia establida entre $\mathscr{F}$-operadores quan $\mathscr{F}$ recorre aquelles 
famílies més estudiades en Teoria de Ramsey. En segon lloc, analitzem quin tipus de propietats de densitat poden tindre els conjunts de la forma $N(x, U)=\left\{n \in \mathbb{N}: T^{n} x \in U\right\}$ i $N(U, V)$ per a un operador hipercíclic dau. De la mateixa manera, classifiquem els operadors hipercíclics d'acord amb estes propietats. En el capítol tres, s'introduix la noció seguent: un operador $T$ en $X$ satisfà la propietat $\mathcal{P}_{\mathscr{F}}$ si per a tot conjunt obert no buit $U$ de $X$, hi ha $x \in X$ tal que $N(x, U) \in \mathscr{F}$. Siga $\overline{\mathcal{B D}}$ la família dels conjunts de $\mathbb{N}$ amb densitat de Banach superior positiva. En primer lloc, generalitzem un resultat de Costakis i Parissis fent ús d'una versió generalitzada del Teorema de Szemerédi, a causa de Bergelson i McCutcheon. Com a consequència obtenim una caracterització d'aquells operadors que satisfan la propietat $\mathcal{P}_{\overline{\mathcal{B D}}}$. Resulta que els operadors tenint la propietat $\mathcal{P}_{\overline{\mathcal{B D}}}$ satisfan un tipus de recurrència que pot ser descrit en termes dels idempotentes essencials de $\beta \mathbb{N}$ (the Stone-Čech compactification of $\mathbb{N}$ ). Es discutix també, el cas dels operadors desplaçament ponderats que satisfan la propietat $\mathcal{P}_{\overline{\mathcal{B D}}}$. D'altra banda, s'obté com a consequència una caracterització dels operadors reiterativament hipercíclics, i.e. operadors per als quals hi ha $x \in X$ tal que per a tot conjunt obert no buit $U$ de $X$, el conjunt $N(x, U) \in \overline{\mathcal{B D}}$.

En el quart capítol ens enfoquem en l'estudi d'un refinament de la noció de hiperciclicidad disjunta. D'una banda, estenem un resultat de Bes, Martin, Peris i Shkarin on afirmem el seguent: 
$B_{w}$ és $\mathscr{F}$-operador si i només si $\left(B_{w}, \ldots, B_{w}^{r}\right)$ és $d-F$, per a tot $r \in \mathbb{N}$, on $B_{w}$ denota un operador desplaçament ponderat en $c_{0}$ o $l_{p},(1 \leq p<\infty)$ i on $\mathscr{F}$ és qualsevol dels filtres més usats en Teoria de Ramsey que contenen estrictament la família dels conjunts cofinitos. D'altra banda, es destaca que este fenomen no té lloc fora del context dels operadors desplaçament ponderats. Per a això es mostra un operador lineal mesclen-te $T$ en un espai de Hilbert tal que $\left(T, T^{2}\right)$ no és $d$-sindético. També s'indaga sobre la relació entre operadors reiterativament hipercíclics i $d-F$ tuplas, per a filtres $\mathscr{F}$ continguts en la família dels conjunts sindéticos. Finalment, examinem quines condicions són necessàries perquè un operador desplaçament ponderat sindético siga reiterativament hipercíclic. 


\section{Acknowledgements}

To everyone except me -the death of your ego is not your death-. Special thanks to Prof. Alfred Peris for his support as supervisor. We also thank Prof. Bernhard Ruf, and Università degli Studi di Milano for the scholarship received during the preparation of this thesis. 


\section{Contents}

1 Introduction 1

1.1 Preliminaries ..................... 1

2 F-operators 13

$2.1 \mathscr{F}$-Criterion and applications . . . . . . . . . . . . 16

2.2 Return sets and density . . . . . . . . . . . 27

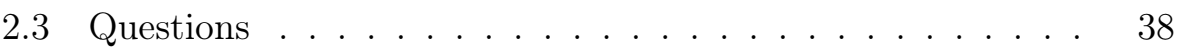

3 Recurrence properties defined via essential idempotents of $\beta \mathbb{N}$

3.1 Adjoints of multiplication operators . . . . . . . . . 47

3.2 Weighted shifts satisfying property $\mathcal{P}_{\overline{\mathcal{B D}}} \ldots \ldots \ldots$

3.2.1 A multiple recurrent operator which is not $\mathcal{E}$-recurrent 49

3.2.2 Further consequences . . . . . . . . . . . . . 51

3.3 A characterization of reiteratively hypercyclic operators . . 57

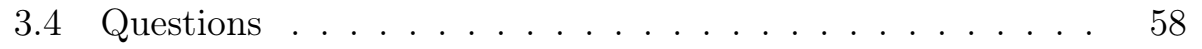

4 Disjoint hypercyclicity along filters 61

4.1 Tuples of powers of weighted shifts . . . . . . . . . 62 
4.1.1 Bilateral weighted shifts . . . . . . . . . 64

4.1.2 Unilateral weighted shifts . . . . . . . . . 71

4.1.2.1 The non-filter case . . . . . . . . . 767

4.2 An $\mathscr{F}$-operator $T$ for which $\left(T, T^{2}\right)$ is not $d-\mathscr{F} \ldots \ldots$

4.3 Reiteratively hypercyclicity vs. $d-\mathscr{F}$ tuples . . . . . . 82

4.4 Moving from syndetic to reiteratively hypercyclic weighted shifts on $c_{0}\left(\mathbb{Z}_{+}\right) \ldots \ldots \ldots \ldots \ldots$

4.5 Questions ........................ 94

Bibliography 


\section{1}

\section{Introduction}

This thesis is concerned with Linear dynamics and is devoted to the study of recurrence properties of linear and bounded operators defined on a real or complex topological vector space $X$, whose topology has a countable basis and is complete.

In the next section we will recall the main objects to deal with later and will mention existing results in the literature, useful for our future work.

\section{$1.1 \quad$ Preliminaries}

Let $\mathcal{L}(X)$ be the algebra of linear and continuous maps on a topological vector space $X$ (in short, operators).

Definition 1. Given an infinite set $A \subseteq \mathbb{N}$, a sequence $\left(T_{n}\right)_{n \in A}$ of continuous maps on $X$ is said universal if there exists $x \in X$ such that the set $\left\{T_{n} x: n \in A\right\}$ is dense in $X$.

In the particular case in which the sequence is composed by the iterates of a single linear operator $T \in \mathcal{L}(X)$, the sequence of operators $\left(T^{n}\right)_{n \in A}$ is 


\section{INTRODUCTION}

said to be hypercyclic, i.e. if there exists $x \in X$ such that $\left\{T^{n} x: n \in A\right\}$ is dense in $X$. Such a vector $x$ is said to be hypercyclic for $T$ with respect to $A \subseteq \mathbb{N}$ and the set of all hypercyclic vectors for $T$ with respect to $A$ is denoted $H C(T, A)$. Whenever $A=\mathbb{N}, T$ is called hypercyclic.

The so-called Hypercyclicity Criterion is of crucial importance in the theory of hypercyclic operators. It was obtained independently by Kitai (29) and by Gethner and Shapiro (22), formulated in two slightly different ways. It provides a sufficient condition for a general operator to be hypercyclic. We give here the formulation given in (17).

Definition 2. We say that $T \in \mathcal{L}(X)$ satisfies the Hypercyclicity Criterion if there exists an increasing sequence of integers $\left(n_{k}\right)_{k}$, two dense sets $D_{1}, D_{2} \subset X$ and a sequence of maps $S_{n_{k}}: D_{2} \longrightarrow X$ such that:

(1) $T^{n_{k}}(x) \rightarrow 0$, for any $x \in D_{1}$

(2) $S_{n_{k}}(y) \rightarrow 0$, for any $y \in D_{2}$

(3) $T^{n_{k}} S_{n_{k}}(y) \rightarrow y$, for any $y \in D_{2}$.

The concept of hypercyclicity is closely related to the notion of transitivity coming from topological dynamics.

Definition 3. The operator $T \in \mathcal{L}(X)$ is topologically transitive if for every pair of non-empty open sets (opene for short) $U, V$ in $X$, the return time set $N(U, V)=\left\{n \in \mathbb{N}: T^{n} U \cap V \neq \emptyset\right\}$ is non-empty.

In fact, both notions are equivalent when we are dealing with an $F$ space, i.e. a complete and metrizable topological vector space, Birkhoff's transitivity theorem (4). Other dynamical properties have been studied in linear dynamics. 
Definition 4. An operator $T \in \mathcal{L}(X)$ is mixing if $N(U, V)$ is cofinite for every opene sets $U, V$ in $X$.

An operator $T \in \mathcal{L}(X)$ is weakly mixing if $T \oplus T$ is hypercyclic on $X \times X$.

It turns out that an operator is weakly mixing if and only if it satisfies the Hypercyclicity Criterion (17).

Obviously, mixing operators are weakly mixing, and these in turn are hypercyclic. Examples of weakly mixing operators being not mixing are not difficult to find. Nevertheless, for a long time all known hypercyclic operators were known to satisfy some version of the Hypercyclicity criterion and the problem of finding a hypercyclic but non-weakly mixing operator turned out to be highly non-trivial, the first example of this kind is due to de la Rosa and Read (33). Later, successive examples of this nature on classical spaces were given by Bayart and Matheron (5).

Definition 5. Let $\mathscr{F}$ be a set of subsets of $\mathbb{Z}_{+}\left(\mathscr{F} \subseteq \mathcal{P}\left(\mathbb{Z}_{+}\right)\right.$for short $)$, we say that $\mathscr{F}$ is a family provided

(I.) $|A|=\infty$ for any $A \in \mathscr{F}$

(II.) $A \subset B$ implies $B \in \mathscr{F}$, for any $A \in \mathscr{F}$.

A family $\mathscr{F}$ is a filter if it is invariant by finite intersections, i.e. $\mathscr{F}$ is a family such that for any $A \in \mathscr{F}, B \in \mathscr{F}$ implies $A \cap B \in \mathscr{F}$.

The notion of filter is closely related to that of partition regular, which in turn will be useful for us in order to identify filters.

Definition 6. ((27), Definition 3.10)

Let $\mathscr{F} \subseteq \mathcal{P}\left(\mathbb{Z}_{+}\right), \mathscr{F}$ is said to be partition regular on $\mathbb{N}$ if for every $A \in \mathscr{F}$ and any finite partition $\left\{A_{1}, \ldots, A_{n}\right\}$ of $A$, there exists some $i=1, \ldots, n$ such that $A_{i} \in \mathscr{F}$. 


\section{INTRODUCTION}

The dual family $\mathscr{F}^{*}$ of $\mathscr{F}$ is defined as

$$
\mathscr{F}^{*}=\{A \subseteq \mathbb{N}: A \cap B \neq \emptyset, \quad \forall B \in \mathscr{F}\}
$$

Lemma 7. (27)

If $\mathscr{F}$ is partition regular then $\mathscr{F}^{*}$ is a filter.

An ultrafilter on $\mathbb{N}$ is a maximal filter, i.e. it is not properly contained in any other filter. Let $\beta \mathbb{N}$ be the Stone-Cech Compactification of $\mathbb{N}$, it is a compact right topological semigroup $(\beta \mathbb{N},+)$. The points of $\beta \mathbb{N}$ are the ultrafilters on $\mathbb{N}$, where each point of $\mathbb{N}$ is identified with a principal ultrafilter in order to obtain an embedding of $\mathbb{N}$ into $\beta \mathbb{N}$. For any $A \subseteq \mathbb{N}$ and $p \in \beta \mathbb{N}$, the closure of $A(\operatorname{cl} A)$ in $\beta \mathbb{N}$ is defined as, $p \in \operatorname{cl} A$ if and only if $A \in p$. Given $p, q \in \beta \mathbb{N}$ and $A \subseteq \mathbb{N}$, the operation $(\mathbb{N},+)$ can be extended to $\beta \mathbb{N}$ by defining: $A \in p+q$ if and only if $\{n \in \mathbb{N}:-n+A \in q\} \in p$, where $-n+A$ denote the set $\{t \in \mathbb{N}: n+t \in A\}$.

Denote by $E(\mathbb{N})=\{p \in \beta \mathbb{N}: p=p+p\}$ the collection of idempotents in $\beta \mathbb{N}$. For details see (27).

Please note that if $\mathscr{F}$ is an ultrafilter, $\mathscr{F}=\mathscr{F}^{*}$. In fact, $\mathscr{F} \subseteq \mathscr{F}^{*}$ obviously follows from the fact that $\mathscr{F}$ is also a filter. Conversely, let $A \in \mathscr{F}^{*}$ and suppose $A \notin \mathscr{F}$, then $A^{c} \in \mathscr{F}$ by theorem 3.6 (27), hence $A \cap A^{c} \neq \emptyset$ which is a contradiction.

We will need the following fact. If $\mathscr{F}=\cup_{\alpha} \mathscr{F}_{\alpha}$, where $\mathscr{F}_{\alpha}$ are ultrafilters, then

$$
A \in \mathscr{F}^{*} \Leftrightarrow A^{c} \notin \mathscr{F} \text {. }
$$

In fact, suppose $A^{c} \in \mathscr{F}$, by hypothesis $A \in \mathscr{F}^{*}$, which implies $A \cap A^{c} \neq$ $\emptyset$, which is a contradiction. Conversely, suppose $A^{c} \notin \mathscr{F}$, then $A^{c} \notin \mathscr{F}_{\alpha}, \forall \alpha$, 
which implies $A \in \mathscr{F}_{\alpha}, \forall \alpha$ by theorem 3.6 (27), hence

$$
A \in \cap_{\alpha} \mathscr{F}_{\alpha}=\cap_{\alpha} \mathscr{F}_{\alpha}^{*}=\mathscr{F}^{*} .
$$

However, the collection of syndetic sets can not be written as a union of ultrafilters.

Let us summarize some families commonly used in Ramsey theory. For a rich source on this subject, see (27).

\section{Definition 8.}

- $\mathcal{J}=\{A \subseteq \mathbb{N}:|A|=\infty\}$, where $|A|$ stands for the cardinality of $A$

- $\Delta=\{A \subseteq \mathbb{N}: B-B \subseteq A$, for some $B \in \mathcal{J}\}$

- JP $=\left\{A \subseteq \mathbb{N}: \exists\left(x_{n}\right)_{n} \subseteq \mathbb{N}, \sum_{n \in F} x_{n} \in A\right.$, for any $\left.F \in P_{f}(\mathbb{N})\right\}$, where $P_{f}(\mathbb{N})=\{A \subset \mathbb{N}:|A|<\infty\}$

- $A$ is syndetic set ( $A \in \mathcal{S}$ for short) if $A$ has bounded gaps, i.e. if $A$ can be enumerated increasingly as $A=\left\{x_{n}: n \in \mathbb{N}\right\}$, then $\max _{n} x_{n+1}-$ $x_{n}<\infty$

- $A$ is thick set ( $A \in \mathcal{T}$ for short) if $A$ contains arbitrarily long intervals, i.e. $\mathcal{T}=\{A \subseteq \mathbb{N}: \forall L>0, \exists n:\{n, n+1, \ldots, n+L\} \subset A\}$

- $A$ is piecewise syndetic set ( $A \in \mathcal{P S}$ for short) if $A$ can be written as the intersection of a thick and a syndetic set.

All of these families can be described using ultrafilter language.

Now, $\Delta, \mathcal{J P}$ and $\mathcal{P S}$ are partition regular families, hence $\mathcal{J}^{*}$ (family of cofinite sets), $\Delta^{*}, \mathcal{J P}^{*}$ and $\mathcal{P S} *$ are filters and

$$
\mathcal{J}^{*} \varsubsetneqq \Delta^{*} \varsubsetneqq \mathcal{J P}^{*} \varsubsetneqq \mathcal{S} \quad \text { as well as } \quad \mathcal{J}^{*} \varsubsetneqq \mathcal{P S}^{*} \varsubsetneqq \mathcal{S} .
$$


See (27), (28), for more details.

Let us recall the notions of asymptotic and Banach density on $\mathbb{N}$.

\section{Definition 9. The asymptotic density:}

The upper and lower asymptotic density are defined respectively by

$$
\begin{aligned}
& \bar{d}(A)=\limsup _{n \rightarrow \infty} \frac{|A \cap\{1,2, \ldots, n\}|}{n} \\
& \underline{d}(A)=\liminf _{n \rightarrow \infty} \frac{|A \cap\{1,2, \ldots, n\}|}{n} .
\end{aligned}
$$

Set $\overline{\mathcal{D}}=\{A \subseteq \mathbb{N}: \bar{d}(A)>0\}$ and $\underline{\mathcal{D}}=\{A \subseteq \mathbb{N}: \underline{d}(A)>0\}$.

The Banach density:

For every real number $s \geq 1$, we define

$$
\alpha^{s}=\limsup _{k \rightarrow \infty}|A \cap[k+1, k+s]|
$$

and

$$
\alpha_{s}=\liminf _{k \rightarrow \infty}|A \cap[k+1, k+s]| .
$$

Each one divided by $s$, tends to a limit, when $s$ tends to infinity. Now, the upper and lower Banach density are defined respectively by

$$
\overline{B d}(A)=\lim _{s \rightarrow \infty} \frac{\alpha^{s}}{s} \quad \text { and } \quad \underline{B d}(A)=\lim _{s \rightarrow \infty} \frac{\alpha_{s}}{s} .
$$

Set $\overline{\mathcal{B D}}=\{A \subseteq \mathbb{N}: \overline{B d}(A)>0\}$ and $\underline{\mathcal{B D}}=\{A \subseteq \mathbb{N}: \underline{B d}(A)>0\}$.

It is a known fact that

$$
\begin{gathered}
\underline{B d}(A) \leq \underline{d}(A) \leq \bar{d}(A) \leq \overline{B d}(A) . \\
\bar{d}(A)+\underline{d}\left(A^{c}\right)=1 .
\end{gathered}
$$

The following diagram shows a hierarchy of all of theses classes of sets in $\mathbb{N}$. 


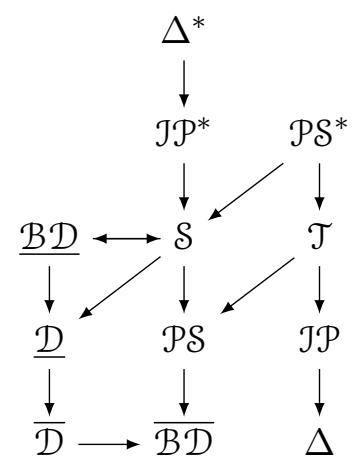

Figure 1

Missing implications in the last figure, are not valid, see (28), (9). Examples of this fact are the following sets.

- $\left\{2^{n}-2^{m}: n, m \in \mathbb{N}, m<n\right\} \in \Delta \backslash \mathcal{J P}$

- $\left\{2^{n}+2 m-1: n, m \in \mathbb{N}, m<n\right\} \in \mathcal{P S} \backslash(\mathcal{S} \cup \mathcal{T})$

- $\{2 n+1: n \in \mathbb{N} \cup\{0\}\} \in \mathcal{S} \backslash\left(\mathcal{J P}^{*} \cup \mathcal{P S}^{*}\right)$

- $\left\{2^{n}+m: n, m \in \mathbb{N}, m<n\right\} \in \mathcal{T} \backslash \mathcal{P S}^{*}$

- $\{2 n: n \in \mathbb{N}\} \backslash\left\{2^{n}-2^{m}: n, m \in \mathbb{N}, m<n\right\} \in \mathcal{J P}^{*} \backslash \Delta^{*}$

- $\{2 n: n \in \mathbb{N}\} \in \Delta^{*}$.

For any choice of real numbers $0 \leq r_{1} \leq r_{2} \leq r_{3} \leq r_{4} \leq 1$, one can find sets $A$ such that

$$
\underline{B d}(A)=r_{1}, \underline{d}(A)=r_{2}, \bar{d}(A)=r_{3}, \overline{B d}(A)=r_{4} .
$$




\section{INTRODUCTION}

This result has been recently announced, and will appear in a joint paper by G. Grekos, R. Jin and L. Mišśk.

We would like to study the hierarchy established between operators whose return time set covers some of the families described in Definition 8 as well as their dual. Recall that for a continuous self map $T$ of $X$ and opene sets $U, V$ of $X$, the return time set from $U$ to $V$ is the set $N(U, V)=\left\{n \in \mathbb{N}: T^{n} U \cap V \neq \emptyset\right\}$. We introduce the following:

Definition 10. Let $T$ be a continuous self map of a topological space $X$. Let $\mathscr{F} \subseteq \mathcal{P}\left(\mathbb{Z}_{+}\right)$be a family. We say that $T$ is an $\mathscr{F}$-map provided $N(U, V) \in \mathscr{F}$ for all $U, V$ opene sets of $X$. If in addtion $T \in \mathcal{L}(X)$, we say $T$ is an $\mathscr{F}$-operator.

The continuous map $T$ is called hereditarily $\mathscr{F}$-map if $(N(U, V) \cap A) \in$ $\mathscr{F}$ for every $U, V$ opene sets of $X$ and each $A \in \mathscr{F}$. In in addition $T \in \mathcal{L}(X)$, we say $T$ is hereditarily $\mathscr{F}$-operator.

The operator $T \in \mathcal{L}(X)$ is said to be $\mathscr{F}$-hypercyclic if $\left(T^{n}\right)_{n \in A}$ is hypercyclic for every $A \in \mathscr{F}$.

The following proposition can be shown in a similar way as Birkhoff's transitivity theorem (see, e.g, (24)). Let $\mathscr{F}$ be a family of subsets of $\mathbb{Z}_{+}$.

Proposition 11. Let $X$ be a separable $F$-space and let $T$ be a continuous map on $X$. The following are equivalent:

i) for every $A \in \mathscr{F}$, there exists $x \in X$ such that $\left\{T^{n} x: n \in A\right\}$ is dense in $X$

ii) $T$ is a $\mathscr{F}^{*}$-map.

In particular, if $T \in \mathcal{L}(X)$ then $T$ is $\mathscr{F}$-hypercyclic if and only if $T$ is an $\mathscr{F}^{*}$-operator. Furthermore, $H C(T, A)$ is a dense $G_{\delta^{-}}$-subset of $X$ for every $A \in \mathscr{F}$. 
Regarding the weakly mixing operators, we quote the following:

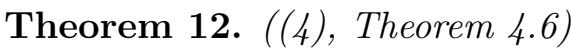

Let $X$ a topological space, and let $T: X \longrightarrow X$ be a continuous map. The following are equivalent:

(1) $T$ is weakly mixing

(2) The sets $N(U, V)$ form a filter basis

(3) For any $L>1$, the $L$-fold product map $T \times \cdots \times T$ is topologically transitive

(4) $N(U, V)$ is thick, for any $U, V$ opene sets in $X$

(5) $N(U, V)-N(U, V)=\mathbb{N}$, for any $U, V$ opene sets in $X$

(6) $N(U, V) \cap N\left(U, V^{\prime}\right) \neq \emptyset$, for any $U, V, V^{\prime}$ opene sets in $X$.

The study of the dynamics of an operator $T$ for return sets $N(U, V)$, where $U$ is a singleton, has already been considered. The following notion was introduced by Quentin Menet (30):

Definition 13. Let $\mathcal{A} \subset \mathcal{P}\left(\mathbb{Z}_{+}\right)$. We say that $\mathcal{A}$ is a frequently hypercyclicity set if it is a family containing a sequence $\left(A_{k}\right)$ of disjoint sets such that for any $j \in A_{k}$, any $j^{\prime} \in A_{k^{\prime}}, j \neq j^{\prime}$, we have

$$
\left|j^{\prime}-j\right| \geq \max \left\{k, k^{\prime}\right\}
$$

Definition 14. Let $X$ be a topological vector space, $T \in \mathcal{L}(X)$ and $\mathcal{A}$ be a frequently hypercyclicity set. We say that $T$ is $\mathcal{A}$-frequently hypercyclic if there exists $x \in X$ such that for any opene set $V \subset X$,

$$
N(x, V)=\left\{n \in \mathbb{N}: T^{n} x \in V\right\} \in \mathcal{A} .
$$

Such a vector $x$ is called an $\mathcal{A}$-frequently hypercyclic vector for $T$. 


\section{INTRODUCTION}

When $\mathcal{A}=\underline{\mathcal{D}}$, the operator $T$ is called frequently hypercyclic, this case was introduced by Bayart and Grivaux, (2), (3).

When $\mathcal{A}=\overline{\mathcal{D}}$, the operator $T$ is called $\mathfrak{U}$-frequently hypercyclic, this case was introduced by Shkarin, (38).

The hierarchy between frequently hypercyclic and $\mathfrak{U}$-frequently hypercyclic operators as well as a full characterization for weighted shift operators have been established by Bayart and Ruzsa (7). It is natural now to consider the case $\mathcal{A}=\overline{\mathcal{B D}}$. This class was introduced by $\mathrm{A}$. Peris as reiteratively hypercyclic operators in a lecture entitled Topologically ergodic operators, during the Conference Function Theory on Infinite Dimensional Spaces IX, held in Madrid in December 2005 (unpublished work).

Finally, let us recall the following notion of chaos for operators. An operator $T \in \mathcal{L}(X)$ is chaotic if it is hypercyclic and has a dense set of periodic points. A point $x \in X$ is periodic if there exists $n \in \mathbb{N}$ such that $T^{n} x=x$. For more details see (4), (24).

Another concept, which we will be interested in is disjoint transitivity. The notion of disjointness was introduced by H. Furstenberg in 1967 (21) for dynamical systems. In 2007, the notion of disjoint hypercyclicity, a strengthening of hypercyclicity concerning a tuple of linear operators, was introduced independently by Bernal (11) and by Bès and Peris (16).

We say that $N$ sequences $\left(T_{1, j}\right)_{j=1}^{\infty}, \ldots,\left(T_{N, j}\right)_{j=1}^{\infty} \in \mathcal{L}(X)$, are $d$-universal if $\left\{\left(T_{1, j} z, \ldots, T_{N, j} z\right): j \in \mathbb{N}\right\}$ is dense in $X^{N}$ for some vector $z \in X$.

\section{Definition 15. (16)}

The $N$-tuple of operators $\left(T_{1}, \ldots, T_{N}\right)$ acting on $X$ is said disjoint transitive ( $d$-transitive for short) if for any $N+1$-tuple $\left(U_{i}\right)_{i=0}^{N}$ of opene sets we 
have

$N_{T_{1}, \ldots, T_{N}}\left(U_{1}, \ldots, U_{N} ; U_{0}\right):=\left\{n \in \mathbb{N}: T_{1}^{-n} U_{1} \cap \cdots \cap T_{N}^{-n} U_{N} \cap U_{0} \neq \emptyset\right\} \neq \emptyset$.

Analogous to the case $N=1$, the $N$-tuple of operators $\left(T_{1}, \ldots, T_{N}\right)$ acting on $X$ is said disjoint mixing ( $d$-mixing for short) if for any $N+1$-tuple $\left(U_{i}\right)_{i=0}^{N}$ of opene sets the set $N_{T_{1}, \ldots, T_{N}}\left(U_{1}, \ldots, U_{N} ; U_{0}\right)$ is cofinite.

If $T_{i}=T^{i}$, for $i=\{1, \ldots, N\}$, then we write $N_{T}$ instead of $N_{T, \ldots, T^{N}}$.

For recent results on disjoint hypercyclicity, see (12), (13), (14), (15), (34) and (37).

The following definition is the Hypercyclicity Criterion for the setting of disjointness (16).

Definition 16. (definition 2.5 (16))

Let $\left(n_{k}\right)_{k}$ be a strictly increasing sequence of positive integers and $\mathscr{F}$ a family on $\mathbb{N}$. We say that $\left(T_{1}, \ldots, T_{N}\right)$ a tuple of operators in $\mathcal{L}(X)$ satisfies $d$-Hypercyclicity Criterion with respect to $\left(n_{k}\right)_{k}$ provided there exists dense subsets $X_{0}, \ldots, X_{N}$ of $X$ and mappings $S_{l, k}: X_{l} \longrightarrow X,(1 \leq l \leq N, k \in \mathbb{N})$ satisfying

(i) $\lim _{k \rightarrow \infty} T_{l}^{n_{k}}(x)=0, \quad$ for any $x \in X_{0}$

(ii) $\quad \lim _{k \rightarrow \infty} S_{l, k}(x)=0, \quad$ for any $x \in X_{l} \quad(1 \leq l \leq N)$

(iii) $\lim _{k \rightarrow \infty}\left(T_{l}^{n_{k}} S_{i, k}-\delta_{i, l} I d_{X_{l}}\right) x=0, \quad$ for any $x \in X_{l} \quad(1 \leq i, l \leq N)$.

We say that $\left(T_{1}, \ldots, T_{N}\right)$ satisfies the $d$-Hypercyclicity Criterion if there exists some sequence $\left(n_{k}\right)_{k}$ for which $(i)$-(iii) are satisfied.

The next Proposition, taken from (16) shows the intimate relationship between $d$-Hypercyclicity Criterion and the notion of $d$-mixing. 
Proposition 17. ((16), Proposition 2.6)

Let $\left(T_{1}, \ldots, T_{N}\right)$ satisfies the d-Hypercyclicity Criterion with respect to a sequence $\left(n_{k}\right)$. Then the tuple of sequences $\left(\left(T_{1}^{n_{k}}\right)_{k=1}^{\infty}, \ldots,\left(T_{N}^{n_{k}}\right)_{k=1}^{\infty}\right)$ is d-mixing. In particular, $\left(T_{1}, \ldots, T_{N}\right)$ is d-hypercyclic.

In Linear dynamics recurrence properties have been frequently studied first in the context of weighted shifts.

Each bilateral bounded weight $w=\left(w_{k}\right)_{k \in \mathbb{Z}}$, induces a bilateral weighted backward shift $B_{w}$ on $X=c_{0}(\mathbb{Z})$ or $l_{p}(\mathbb{Z})(1 \leq p<\infty)$, given by $B_{w} e_{k}:=$ $w_{k} e_{k-1}$, where $\left(e_{k}\right)_{k \in \mathbb{Z}}$ denotes the canonical basis of $X$ and $\left(e_{k}^{*}\right)_{k \in \mathbb{Z}}$ the associated sequence of coordinate functionals.

Similarly, each unilateral bounded weight $w=\left(w_{n}\right)_{n \in \mathbb{Z}_{+}}$induces a unilateral weighted backward shift $B_{w}$ on $X=c_{0}\left(\mathbb{Z}_{+}\right)$or $l_{p}\left(\mathbb{Z}_{+}\right)(1 \leq p<\infty)$, given by $B_{w} e_{n}:=w_{n} e_{n-1}, n \geq 1$ with $B_{w} e_{0}:=0$, where $\left(e_{n}\right)_{n \in \mathbb{Z}_{+}}$denotes the canonical basis of $X$ and $\left(e_{n}^{*}\right)_{n \in \mathbb{Z}_{+}}$the associated sequence of coordinate functionals.

Finally, we quote two results of (14) that we will use later.

Theorem 18. (14)

Let $B_{w}$ a weighted backward shit on $c_{0}$ or $l_{p},(1 \leq p<\infty)$, then $B_{w}$ is mixing if and only if $\left(B_{w}, \ldots, B_{w}^{r}\right)$ is d-mixing, for any $r \in \mathbb{N}$.

Theorem 19. ((14), Theorem 3.8)

There exists $T \in \mathcal{L}\left(l_{2}\right)$ such that $T$ is mixing and $\left(T, T^{2}\right)$ is not d-mixing. 


\section{$\mathscr{F}$-operators}

Despite of the different size of the classes of sets exhibited in Figure 1, one can see that restricted to $N_{T}(U, V)$, where $U$ and $V$ are opene sets in $X$, equivalence between certain classes of operators can be obtained.

Proposition 20. Let $T$ be a continuous map on a separable $F$-space $X$ and let $\mathscr{F} \subseteq \mathcal{P}\left(\mathbb{Z}_{+}\right)$be a partition regular family. The following are equivalent:

i) $T$ is an $\mathscr{F}^{*}$-map

ii) $T$ is hereditarily $\mathscr{F}^{*}$-map

iii) for any $A \in \mathscr{F}$, there exists $x \in X$ such that $\left\{T^{n} x: n \in A\right\}$ is dense in $X$

iv) $T$ is a hereditarily $\mathscr{F}$-map.

Proof. i) $\Rightarrow$ ii) Obvious because $\mathscr{F}^{*}$ is a filter, since $\mathscr{F}$ is partition regular, see (27).
ii) $\Rightarrow$ i) Obvious.
i) $\Leftrightarrow$ iii) By Proposition 11
i) $\Rightarrow$ iv) Denote $I=\{p \in \beta \mathbb{N}: \forall A \in p, A \in \mathscr{F}\}$. 
Let $A \in \mathscr{F}$, by Theorem $3.11(27)$ and using the fact that $\mathscr{F}$ is partition regular, we have there exists $\widetilde{p} \in I$ such that $A \in \widetilde{p}$. Hence $I \neq \emptyset$

On the other hand, $N(U, V) \in p$ for every $p \in I$ and $U, V$ opene sets. Suppose the contrary, $N(U, V) \notin q$ for some $q \in I$. By Theorem 3.6 e) (27), we have $N(U, V)^{c} \in q$, which implies $N(U, V)^{c} \in \mathscr{F}$, but by i) $N(U, V) \in$ $\mathscr{F}^{*}$, hence $N(U, V) \cap N(U, V)^{c} \neq \emptyset$, which is a contradiction.

Concluding, for any $A \in \mathscr{F}$, pick $\widetilde{p} \in I$ such that $A \in \widetilde{p}$, but $N(U, V) \in \widetilde{p}$ for every opene sets $U, V$, which implies $N(U, V) \cap A \in \widetilde{p}$ for every opene sets $U, V$. Hence, $N(U, V) \cap A \in \mathscr{F}$ for every opene sets $U, V$.

iv) $\Rightarrow$ i) Obvious.

Notice that the case when $\mathscr{F}=\{A \subseteq \mathbb{N}:|A|=\infty\}$ in Proposition 20 and $T \in \mathcal{L}(X)$, extends the well known fact that $T$ is mixing if and only if it is hereditarily hypercyclic with respect to the full sequence $(n)$.

Now, in the linear case, we have the following

Proposition 21. Let $X$ a topological vector space, $T \in \mathcal{L}(X)$ and $\mathscr{F}$ partition regular. The following are equivalent:

i) $T \in \mathscr{F}^{*}$-operator

ii) $N(U, W) \in \mathscr{F}^{*}$ and $N(W, V) \in \mathscr{F}^{*}$, for every $U, V$ opene sets and $W$ any open neighborhood of 0.

Proof. i) $\Rightarrow$ ii) Obvious

ii) $\Rightarrow$ i) Let $U, V$ opene sets, there exist $U^{\prime}, V^{\prime}$ opene sets and $W^{\prime}$ open neighborhood of 0 such that,

$$
U^{\prime}+W \subseteq U \quad V^{\prime}+W \subseteq V
$$

Now, by linearity of the operator, we have $N\left(U^{\prime}, W\right) \cap N\left(W, V^{\prime}\right) \subseteq$ $N(U, V)$ and i) follows from ii) because $\mathscr{F}^{*}$ is a filter. 
The following is a known result.

Proposition 22. Let $T$ be a topological vector space and $T \in \mathcal{L}(X)$. The following are equivalent:

i) $T$ is an $\mathcal{S}$-operator

ii) $T$ is a hereditarily syndetic operator

iii) $N(U, W) \in \mathcal{S}$ and $N(W, V) \in \mathcal{S}$ for any $U, V$ opene sets and $W$ an open neighborhood of 0 .

Proof. i) $\Rightarrow$ ii) see the proof of [(1), Proposition 4.6]. Here the authors use strongly the linearity of the operator.

ii) $\Rightarrow$ i) Obvious

i) $\Rightarrow$ iii) Obvious

iii) $\Rightarrow$ i) This is a well known fact, see exercise 2.5.4 ii) in (24). Indeed, it suffices to show $N(U, W) \cap N(W, V)$ is syndetic for every $U, V$ opene sets and $W$ open neighborhood of 0 . Then, we can conclude because $N\left(U^{\prime}, W\right) \cap$ $N\left(W, V^{\prime}\right) \subseteq N(U, V)$ whenever $U^{\prime}+W \subseteq U$ and $V^{\prime}+W \subseteq V$.

Syndetic operators are also known as topologically ergodic operators.

Corollary 23. Let $T \in \mathcal{L}(X)$, where $X$ is a separable $F$-space. The following are equivalent:

i) $T \in \mathcal{S}$-operator

ii) $T$ is hereditarily syndetic operator

iii) $T \in \mathcal{P S}^{*}$-operator

iv) $N(U, W) \in \mathcal{S}$ and $N(W, V) \in \mathcal{S}$ for any $U, V$ opene sets and $W$ any open neighborhood of 0

v) $\left(T^{n}\right)_{n \in B}$ is hypercyclic for any $B \in \mathcal{P S}$. 
Proof. By Proposition 22 we have that $(i),(i i)$ and $(i v)$ are equivalents. On the other hand, by Proposition 20 we have that (iii) and $(v)$ are equivalents, since $\mathcal{P S}$ is partition regular. Finally, (ii) implies (iii) because each $\mathcal{P S}$ set can be written as the intersection of a thick set and a syndetic one. Obviously (iii) implies (i).

Concluding, hence it is immediate that a $\mathcal{P} \mathcal{S}^{*}$-map is a $\mathcal{S}$-map. Now we may wonder whether in this context these two notions of $\mathscr{F}$-maps coincide: is each $\mathcal{S}$-map a $\mathcal{P S}^{*}$-map? If this were the case, then each $\mathcal{S}$-map is a $\mathcal{T}$-map (i.e. weakly mixing), and it is a well known fact that irrational rotations of the unit circle are syndetic and not weakly mixing maps. Hence, not every $\mathcal{S}$-map is $\mathcal{P S}^{*}$-map. However adding linearity to the map, the desired equivalence between these two classes of operators can be obtained. In broad terms, we can affirm that linearity of the operator comes to fill the gap caused by the algebraic poverty of the collection of syndetic sets.

\section{$2.1 \quad \mathscr{F}$-Criterion and applications}

The aim of this section is to study what happen with the hierarchy established for the classes $\mathscr{F}=\mathcal{S}, \mathfrak{J P}^{*}, \Delta^{*}$, this time at the level of operators. For example, is $T$ a $\Delta^{*}$-operator if and only if it is a syndetic operator? In order to be able to answer this question, it would be nice to have at hand a characterization of $\mathscr{F}$-weighted backward shifts on $l_{p}$ or $c_{0}$, via a condition relying completely on the weight. If we take a look, for example, at the characterization of chaotic weighted backward shifts on $l_{p}\left(\mathbb{Z}_{+}\right)$, Theorem 6.12 (4), one realizes that the application of the Chaoticity criterion, Theorem 6.10 (4) plays a crucial role. Inspired on this fact, we present what we 
call the $\mathscr{F}$-Criterion, which gives suffcient conditions for an operator to be an $\mathscr{F}$-operator. With the aid of this criterion we will be able to obtain the desired characterization of $\mathscr{F}$-weighted backward shifts on $l_{p}$ or $c_{0}$.

Let us recall the definition of limits along a collection of sets.

Definition 24 ( $\mathscr{F}$-limit).

$$
\mathscr{F}-\lim _{n} T^{n}(x):=y
$$

if and only if for every opene neighborhood $V$ of $y$,

$$
\left\{n \in \mathbb{N}: T^{n}(x) \in V\right\} \in \mathscr{F} .
$$

Proposition 25 ( $\mathscr{F}$-Criterion).

Suppose $X$ is a topological vector space, and that there exist $D_{1}, D_{2}$ dense sets in $X$, a sequence of maps $S_{n}: D_{2} \rightarrow X$ and a filter $\mathscr{F}$ on $\mathbb{N}$ such that

1. $\mathscr{F}-\lim _{n} T^{n}(x)=0$ for every $x \in D_{1}$

2. $\mathscr{F}-\lim _{n} S_{n}(y)=0$ for every $y \in D_{2}$

3. $\mathscr{F}-\lim _{n} T^{n} S_{n}(y)=y$ for every $y \in D_{2}$

Then $T$ is a $\mathscr{F}$-operator, that is, $N(U, V) \in \mathscr{F}$ for every $U, V$ opene sets.

Proof. Let $U, V$ opene sets. Pick $x \in D_{1} \cap U$ and $y \in D_{2} \cap V$.

$$
\mathscr{F}-\lim _{n}\left(x+S_{n} y\right)=x \in U
$$

$\mathscr{F}-\lim _{n}\left(T^{n}\left(x+S_{n} y\right)\right)=\mathscr{F}-\lim _{n} T^{n} x+\mathscr{F}-\lim _{n} T^{n} S_{n} y=y \in V$.

Hence,

$$
\begin{gathered}
A_{1}=\left\{n \in \mathbb{N}: T^{n}\left(x+S_{n} y\right) \in V\right\} \in \mathscr{F} \\
A_{2}=\left\{n \in \mathbb{N}: x+S_{n} y \in U\right\} \in \mathscr{F} .
\end{gathered}
$$


Please note that $A_{1} \cap A_{2} \subseteq N(U, V)$. Now, $\mathscr{F}$ is a filter, hence intersection of $\mathscr{F}$-sets is again $\mathscr{F}$-set, then $A_{1} \cap A_{2} \in \mathscr{F}$ and finally by Definition 5 II, we conclude that $N(U, V) \in \mathscr{F}$.

Corollary 26. $\mathscr{F}$-criterion holds in particular when $\mathscr{F}=\mathcal{P S}^{*}, \Delta^{*}, \mathcal{J P}^{*}, \overline{\mathcal{D}}^{*}$ and $\overline{\mathcal{B D}}^{*}$.

Recall that $P_{f}(\mathbb{Z})=\{A \subset \mathbb{Z}:|A|<\infty\}$. Denote, for $M>0$ and $j \in \mathbb{Z}$

$$
\begin{gathered}
A_{M, j}:=\left\{n \in \mathbb{N}: \prod_{i=j+1}^{j+n}\left|w_{i}\right|>M\right\} \\
\bar{A}_{M, j}=\left\{n \in \mathbb{N}: \frac{1}{\prod_{i=j-n+1}^{j}\left|w_{i}\right|}>M\right\} .
\end{gathered}
$$

In the case $j=0$, we just write $A_{M}, \bar{A}_{M}$ instead of $A_{M, 0}, \bar{A}_{M, 0}$ respectively.

Now, as an application of the $\mathscr{F}$-criterion we have the following characterization of $\mathscr{F}$-weighted backward shifts.

Proposition 27. Consider the family $\mathscr{F}$ and $B_{w}$ a bilateral weighted backward shift on $X=c_{0}(\mathbb{Z})$ or $l_{p}(\mathbb{Z})(1 \leq p \leq \infty)$, then the following are equivalent:

i) $N(U, V) \in \mathscr{F}$ for every $U, V$ opene sets in $X$

ii) For every $M>0$ and $F \in P_{f}(\mathbb{Z})$,

$$
\cap_{j \in F} A_{M, j} \in \mathscr{F}, \quad \cap_{j \in F} \bar{A}_{M, j} \in \mathscr{F} .
$$

In addition, if $\mathscr{F}$ is a filter then $i i)$ is equivalent to

$$
A_{M, j} \in \mathscr{F}, \quad \bar{A}_{M, j} \in \mathscr{F}
$$

for every $M>0, j \in \mathbb{Z}$. 
Proof. $i) \Rightarrow$ ii) Let $M>0, F \in P_{f}(\mathbb{Z})$ and $R>|F|$ such that $M R>1$. Consider the opene sets

$$
\begin{gathered}
A_{R}=\left\{x \in X:\left|e_{j}^{*}(x)\right|>1 / R, \forall j \in F\right\} \cap\{x \in X:\|x\|<1\} \\
V=\left\{x \in X:\left\|x-\sum_{j \in F}(M+1) e_{j}\right\|<\frac{1}{M R}\right\} .
\end{gathered}
$$

Consider the set $G=\left\{m \in N\left(A_{R}, V\right): j-m \notin F, \forall j \in F\right\}$, then $G$ is cofinite in $N\left(A_{R}, V\right)$, i.e. $N\left(A_{R}, V\right) \backslash G$ is a finite set.

Let $m \in G$. Pick $x \in A_{R}$ such that $B_{w}^{m} x \in V$. Then,

$$
\begin{gathered}
\left|\prod_{i=j+1}^{j+m} w_{i} x_{j+m}-(M+1)\right|<\frac{1}{M R}<1, \quad \forall j \in F . \\
\left|\prod_{i=t+1}^{t+m} w_{i} x_{t+m}\right|<\frac{1}{M R}, \quad \forall t \notin F .
\end{gathered}
$$

We get by 2.1.1),

$$
\prod_{i=j+1}^{j+m}\left|w_{i}\right|>\prod_{i=j+1}^{j+m}\left|w_{i} x_{j+m}\right|>M, \quad \forall j \in F .
$$

Thus, $m \in \cap_{j \in F} A_{M, j}$. Hence, $G \subseteq \cap_{j \in F} A_{M, j}$.

On the other hand, we get by 2.1.2,

$$
\prod_{i=j-m+1}^{j}\left|w_{i} x_{j}\right|<\frac{1}{M R}
$$

for any $j \in F$. Hence,

$$
\prod_{i=j-m+1}^{j}\left|w_{i}\right| \frac{1}{R}<\prod_{i=j-m+1}^{j}\left|w_{i} x_{j}\right|<\frac{1}{M R},
$$


for any $j \in F$. Thus, $\prod_{i=j-m+1}^{j}\left|w_{i}\right|<\frac{1}{M}$ for any $j \in F$, i.e. $m \in \cap_{j \in F} \bar{A}_{M, j}$. Hence, $G \subseteq \cap_{j \in F} \bar{A}_{M, j}$.

We can conclude, since $G \in \mathscr{F}$. This follows from the fact that $G$ is cofinite in $N\left(A_{R}, V\right)$ and by hypothesis $N\left(A_{R}, V\right) \in \mathscr{F}$.

ii $\Rightarrow$ i) Consider the filter generated by $\left\{A_{M, j}: M>0, j \in \mathbb{N}\right\}$. Denote it by $\overline{\mathscr{F}}$. Obviously $\overline{\mathscr{F}} \subseteq \mathscr{F}$. It suffices apply $\mathscr{F}$-criterion to the filter $\overline{\mathscr{F}}$. Let $D=D_{1}=D_{2}$ the set of all finitely supported vectors, and $S_{w}$ be the forward shift defined on $D$ by

$$
S_{w}\left(e_{i}\right):=\frac{1}{w_{i+1}} e_{i+1} .
$$

Set $S_{n}:=S_{w}^{n}$. Obviously 3 . in the $\mathscr{F}$-criterion holds. It remains to verify 1. and 2 .

Let $1 \leq p<\infty, \epsilon>0, V_{\varepsilon}:=\left\{x \in l^{p}(\mathbb{N}):\|x\|<\varepsilon\right\}$.

First, we must show that $\left\{n \in \mathbb{N}: T^{n} y \in V_{\varepsilon}\right\} \in \overline{\mathscr{F}}$, for every $y \in D$. So, let $y \in D$, without loss of generality, we can suppose $y=\left(\ldots, 0, y_{-m}, \ldots, y_{m}, 0, \ldots\right)$ with $\prod_{k=-m}^{m} y_{k} \neq 0$. Then,

$$
T^{n} y=(\ldots, 0, \underbrace{y_{-m} \prod_{i=-m-n+1}^{-m} w_{i}}_{(-m-n)-\text { position }}, \ldots, \underbrace{y_{m} \prod_{i=m-n+1}^{m} w_{i}}_{(m-n)-\text { position }}, 0, \ldots) .
$$

Let $M_{j}=y_{j}(2 m)^{1 / p} / \epsilon$ with $j=-m, \ldots, m$ and $M=\max _{j=-m, \ldots, m} M_{j}$. By hypothesis $\cap_{j=-m}^{m} \bar{A}_{M, j} \in \overline{\mathscr{F}}$. Pick $n \in \cap_{j=-m}^{m} \bar{A}_{M, j}$, then we have

$$
\left\|T^{n} y\right\|^{p}=\sum_{j=-m}^{m}\left|y_{j} \prod_{i=j-n+1}^{j} w_{i}\right|^{p}<\sum_{j=-m}^{m}\left|y_{j}\right|^{p}\left(\frac{\varepsilon}{y_{j}(2 m)^{1 / p}}\right)^{p}<\varepsilon^{p},
$$

which implies

$$
\cap_{j=-m}^{m} \bar{A}_{M, j} \subseteq\left\{n \in \mathbb{N}: T^{n} y \in V_{\varepsilon}\right\}
$$


Consequently,

$$
\left\{n \in \mathbb{N}: T^{n} y \in V_{\varepsilon}\right\} \in \overline{\mathscr{F}} \subseteq \mathscr{F} .
$$

Second, we must show that $\left\{n \in \mathbb{N}: S_{n} y \in V_{\epsilon}\right\} \in \overline{\mathscr{F}}$, for every $y \in D$. Let $y \in D$, so suppose as before that $y=\left(\ldots, 0, y_{-m}, \ldots, y_{m}, 0, \ldots\right)$ with $\prod_{k=-m}^{m} y_{k} \neq 0$.. Then,

$$
S_{n}(y)=S_{w}^{n}(y)=(\ldots, 0, \underbrace{\frac{y_{-m}}{\prod_{i=-m+1}^{-m+n} w_{i}}}_{(n-m)-\text { position }}, \ldots, \underbrace{\frac{y_{m}}{\prod_{i=m+1}^{m+n} w_{i}}}_{(n+m)-\text { position }}, 0, \ldots) .
$$

Let $M_{j}$ and $M$ as before. By hypothesis $\cap_{j=-m}^{m} A_{M, j} \in \overline{\mathscr{F}}$. Pick $n \in$ $\cap_{j=-m}^{m} A_{M, j}$, then we have

$$
\left\|S_{n} y\right\|^{p}=\sum_{j=-m}^{m}\left|\frac{y_{j}}{\prod_{i=1}^{n} w_{i}}\right|^{p}<2 m \varepsilon^{p} / 2 m<\varepsilon^{p},
$$

which implies

$$
\cap_{j=-m}^{m} A_{M, j} \subseteq\left\{n \in \mathbb{N}: S_{n} y \in V_{\varepsilon}\right\} .
$$

Consequently,

$$
\left\{n \in \mathbb{N}: S_{n} y \in V_{\varepsilon}\right\} \in \overline{\mathscr{F}} \subseteq \mathscr{F} .
$$

This completes the proof of $i i) \Rightarrow i)$. The same proof works for $l^{\infty}(\mathbb{Z})$ and $c_{0}(\mathbb{Z})$ taking $\left(M_{j}\right)_{j=0}^{m}$ appropriately.

The family $\mathscr{F} \subseteq \mathcal{P}\left(Z_{+}\right)$is said to be shift invariant if $(A+i) \cap \mathbb{N} \in \mathscr{F}$, for every $i \in \mathbb{Z}$, whenever $A \in \mathscr{F}$.

The unilateral version of Proposition 27 follows the same sketch of proof.

Proposition 28. Consider the family $\mathscr{F}$ and $B_{w}$ an unilateral weighted backward shift on $X=c_{0}\left(\mathbb{Z}_{+}\right)$or $l_{p}\left(\mathbb{Z}_{+}\right)(1 \leq p \leq \infty)$, then the following are equivalent: 
i) $N(U, V) \in \mathscr{F}$ for every $U, V$ opene sets in $X$

ii) $N\left(B_{X}(0,1), V\right) \in \mathscr{F}$ for every $V$ opene set in $X$

iii) $\cap_{j \in F} A_{M, j} \in \mathscr{F}$, for every $M>0$ and $F \in P_{f}\left(\mathbb{Z}_{+}\right)$.

In addition,

- if $\mathscr{F}$ is shift invariant then iii) is equivalent to $A_{M} \in \mathscr{F}$, for every $M>0$.

In particular, this holds for the family of syndetic sets.

- if $\mathscr{F}$ is a filter then iii) is equivalent to $A_{M, j} \in \mathscr{F}$, for every $M>$ $0, j \in \mathbb{Z}_{+}$.

Proof. It suffices to show, whenever $\mathscr{F}$ is a shift invariant family that $i i i)$ is equivalent to $A_{M} \in \mathscr{F}$, for every $M>0$. It suffices to prove one implication, since the other one is obvious.

Let $M>0, F \in P_{f}\left(\mathbb{Z}_{+}\right)$. Denote $N=\max \{j: j \in F\}$. By hypothesis the weight is bounded, then there exists $k \in \mathbb{N}$ such that $\left|w_{i}\right|<k$, for every $i \in \mathbb{Z}_{+}$. Enumerate $A_{M k^{N}}$ as $\left(n_{i}\right)_{i \in \mathbb{N}}$, then we have $\prod_{s=0}^{n_{i}}\left|w_{s}\right|>M k^{N}$, which implies

$$
\prod_{s=j}^{n_{i}-(N-j)}\left|w_{s}\right|>\frac{M k^{N}}{k^{N}}=M, \quad \forall i \in \mathbb{Z}_{+}, j \in F
$$

hence, $n_{i}-N \in A_{M, j}$ for every $i \in \mathbb{Z}_{+}$and $j \in F$, in other words,

$$
A_{M k^{N}}-N \subseteq \cap_{j \in F} A_{M, j} .
$$

But $\mathscr{F}$ is a shift invariant family, which implies iii).

Corollary 29. Let $\mathscr{F}$ be a filter, $m \in \mathbb{N}$ and let $B_{w}$ be a bilateral weighted backward shift on $X=l_{p}(\mathbb{Z})$ or $c_{0}(\mathbb{Z})$. Then the following are equivalent:

i) $A_{M, j} \in l \mathscr{F}$ and $\bar{A}_{M, j} \in l \mathscr{F}$, for each $1 \leq l \leq m, M>0$ and $j \in \mathbb{Z}$

ii) $B_{w} \oplus B_{w}^{2} \oplus \ldots \oplus B_{w}^{m}$ is an $\mathscr{F}$-operator on $X^{m}$. 
Proof. By Proposition 27, we have that $A_{M, j} \in l \mathscr{F}$ and $\bar{A}_{M, j} \in l \mathscr{F}$, for any $M>0, j \in \mathbb{Z}$ and any $1 \leq l \leq m$ is equivalent to $N_{B_{w}^{l}}(U, V) \in \mathscr{F}$ for any $U, V$ opene sets in $X$ and $1 \leq l \leq m$. On the other hand, condition $i i)$ is equivalent to $\cap_{l=1}^{m} N_{B_{w}^{l}}\left(U_{l}, V_{l}\right) \in \mathscr{F}$, for any pairs of finite collection of opene sets $\left(U_{l}, V_{l}\right)_{l=1}^{m}$. The conclusion follows since $\mathscr{F}$ is a filter.

Please observe that if one is interested in keeping the same hierarchy between families $\mathscr{F} \subseteq \mathcal{P}\left(\mathbb{Z}_{+}\right)$that appear in Figure 1, but for the corresponding classes of $\mathscr{F}$-operators, clearly the possibility of being able to apply a result in the vein of Proposition 28 is crucial.

Remark 30. When $\mathscr{F}$ is not a shift invariant family of sets, there are two natural ways of building a shift invariant family from it,

$$
\begin{aligned}
& \mathscr{F}_{+}:=\cup_{k \in \mathbb{Z}} \mathscr{F}+k \\
& \mathscr{F}_{\bullet}:=\cap_{k \in \mathbb{Z}} \mathscr{F}+k .
\end{aligned}
$$

Please note that for any $A \subseteq \mathbb{N}$ the following holds

$$
A \in\left(\mathscr{F}^{*}\right) . \Leftrightarrow A \in\left(\mathscr{F}_{+}\right)^{*} .
$$

Let us see some applications of the $\mathscr{F}$-criterion.

Proposition 31. There exists a syndetic but not $\mathcal{J P}^{*}$-weighted backward shift in $X=c_{0}\left(\mathbb{Z}_{+}\right)$or $l_{p}\left(\mathbb{Z}_{+}\right),(1 \leq p \leq \infty)$.

Proof. Consider the set

$$
B=\left\{\sum_{n \in F} 2^{2 n}: F \in P_{f}(\mathbb{N})\right\}
$$


Obviously $B \in \mathcal{J P}$, hence $B^{c} \notin \mathcal{J P}^{*}$ by (1.1.1). Enumerate $B=\left(b_{n}\right)_{n}$. Then define the weight $w=\left(w_{m}\right)_{m=0}^{\infty}$ as follows

$$
w=(2, \ldots, 2, \underbrace{\frac{1}{2^{b_{1}}}}_{w_{b_{1}}}, 2, \ldots, 2, \underbrace{\frac{1}{2^{b_{2}-b_{1}-1}}}_{w_{b_{2}}}, 2, \ldots, 2, \underbrace{\frac{1}{2^{b_{3}-b_{2}-1}}}_{w_{b_{3}}}, 2, \ldots) .
$$

Now, $A_{1}:=\left\{n \geq 0: \prod_{i=0}^{n} w_{i}>1\right\}=B^{c}$, hence $B_{w}$ is not $\mathcal{J P}^{*}$-operator by Propositon 28. On the other hand, $B^{c} \in \mathcal{P S}^{*}$, see list of sets with no extra properties in (9), and observe that $A_{2^{j}}:=\left\{n \geq 0: \prod_{i=0}^{n} w_{i}>\right.$ $\left.2^{j}\right\}=\left(\bigcup_{i=0}^{j} B+i\right)^{c}=\bigcap_{i=0}^{j}(B+i)^{c} \in \mathcal{P S}^{*}$ since $\mathcal{P S}^{*}$ is a filter and $(B+i)^{c} \in \mathcal{P S}^{*}$ for any $i \in\{0, \ldots, j\}$. In fact, let $i \in\{0, \ldots, j\}$, then $B^{c} \in \mathcal{P S}^{*}$ implies $B \notin \mathcal{P S}$ by 1.1.1), which in turn implies $(B+i) \notin \mathcal{P S}$ otherwise $B+i-i=B \in \mathcal{P S}$ because of the shift invariance of $\mathcal{P S}$, hence again by 1.1.1) we have $(B+i)^{c} \in \mathcal{P S}^{*}$. Hence by Propositon 28, $B_{w}$ is $\mathcal{P S}^{*}$-operator, or equivalently a syndetic operator.

Proposition 32. There exists a weighted backward shift operator on $X=$ $c_{0}\left(\mathbb{Z}_{+}\right)$or $l_{p}\left(\mathbb{Z}_{+}\right),(1 \leq p \leq \infty)$, that is $\mathcal{J P}^{*}$ but not a $\Delta^{*}$-operator.

Proof. Consider a set $B$ in $\mathbb{N}$ enumerated increasingly as $\left(b_{n}\right)_{n}$ such that it contains a subsequence $\left(b_{n_{k}}\right)_{k}$ satisfying condition

$$
b_{n_{k}+1}-b_{n_{k}} \rightarrow \infty \text {. }
$$

Define the weight $w=\left(w_{m}\right)_{m=0}^{\infty}$ as in 2.1.4. Observe that condition 2.1.5 assure at least hypercyclicity of $B_{w}$. Let us study which additional conditions must satisfy $B$ in such a way that $B_{w}$ be $\mathrm{JP}^{*}$, but non $\Delta^{*}$ operator. As before $\left\{n \geq 0: \prod_{i=0}^{n} w_{i}>1\right\}=B^{c}$, so it would be desirable that $B \in \Delta$, i.e., $B^{c} \notin \Delta^{*}$ by 11.1 .1 , and this will imply $B_{w} \notin \Delta^{*}$-op.

On the other hand, condition

$$
\bigcup_{i=0}^{n} B+i \notin \mathcal{J P}
$$


for any $n \in \mathbb{N}$, implies $B_{w} \in \mathcal{J P}^{*}$-op, since $\left(\bigcup_{i=0}^{n} B+i\right)^{c} \in \mathcal{J P}^{*}$ for any $n \in \mathbb{N}$. Now, since $\mathcal{J P}$ is partition regular, condition (2.1.6) is obtained if $B \notin \mathcal{J P}+$ and this in turn is equivalent to $B^{c} \in\left(\mathcal{J P}^{*}\right)$. by (1.1.1) and $(2.1 .3)$. Now, an obvious modification in the proof of Theorem 2.11, 1) (8) assures us the existence of a set $E \in\left(\mathcal{J P}^{*}\right)$. which is not $\bigcup_{n \in \mathbb{N} \cup\{0\}}\left(\Delta^{*}+n\right)$-set in $\mathbb{N}$, hence not $\Delta^{*}$-set. Thus, $E^{c}$ satisfies condition (2.1.5), and setting $B=E^{c}$ we are done.

Evidently, every mixing operator is a $\Delta^{*}$-operator but the converse is not true. In Proposition 33 below we construct a non mixing weighted backward shift very close to being mixing.

Proposition 33. There exists a $\left(\Delta^{*}\right)$.-weighted backward shift in $X=$ $c_{0}\left(\mathbb{Z}_{+}\right)$or $l_{p}\left(\mathbb{Z}_{+}\right),(1 \leq p \leq \infty)$ which is not mixing.

Proof. Firstly, let us define what we call $\Delta_{k}$-set. $A \subseteq \mathbb{N}$ is $\Delta_{k}$-set if there exists a finite sequence of $k$ elements, $\left\{x_{1}, x_{2}, \ldots, x_{k}\right\} \subset \mathbb{N}$ such that $x_{j} \in$ $x_{i}+A$ for every $1 \leq i<j \leq k$.

For each $k \in \mathbb{N}$, every $\Delta_{k}$-set contains a $\Delta_{k-1}$-set, hence we have the following chain of implications

$$
\Delta_{2}^{*} \longrightarrow \Delta_{3}^{*} \longrightarrow \ldots \longrightarrow \Delta_{k}^{*} \longrightarrow \ldots \longrightarrow \Delta_{\infty}^{*}=\Delta^{*}
$$

On the other hand, one can easily see that for each $k \in \mathbb{N}$ one can find a cofinite set which is not $\Delta_{k}^{*}$-set. So, these classes $\left(\Delta_{k}^{*}\right)$ are not good candidates in order to obtain a characterization of mixing operators, and we have no choice but moving till $\Delta^{*}$.

Now, $\Delta^{*}$-sets are not shift invariant $\left(2 \mathbb{N} \in \Delta^{*}\right.$, but $\left.2 \mathbb{N}+1 \notin \Delta^{*}\right)$, then it makes sense consider the translates of $\Delta^{*}$-sets, clearly the largest class of sets we can build with these translates in order to be at the same time closest to cofinite sets are those sets contained in every translate, i.e. the 
$\cap_{k \in \mathbb{Z}_{+}}\left(\Delta^{*}+k\right)$-sets. On the other hand, $\Delta$-sets are infinite, consequently cofinite sets are $\Delta^{*}$-sets and the same with any translate of $\Delta^{*}$-sets. Concluding, mixing operators are $\left(\Delta^{*}\right)$.operators, and one could wonder if the reverse holds.

In what follows let us show a $\left(\Delta^{*}\right)$., but not mixing weighted backward shift on $X$, hence not chaotic.

Let us consider the weight $w=\left(w_{n}\right)_{n=0}^{\infty}$,

$$
w=\left(2,1 / 2,2,2,1 / 2^{2}, 2,2,2,1 / 2^{3}, \ldots\right) .
$$

Obviously $B_{w}$ is not mixing since is not satisfied $\lim _{n} \prod_{i=1}^{n} w_{i}=\infty$.

Set $A=\left\{n \in \mathbb{N}: w_{n}<1\right\}$. In order to see that $B_{w}$ is $\left(\Delta^{*}\right)_{\bullet}$-op, by proposition 28 it suffices to prove that

$$
\left(\bigcup_{i=0}^{n} A+i\right)^{c} \in\left(\Delta^{*}\right)
$$

for any $n \in \mathbb{N}$, since $\left(\Delta^{*}\right)$ • is a filter.

Let $n \in \mathbb{N}$ and suppose $\bigcup_{i=0}^{n} A+i$ is a $\Delta$-set. Hence, there exists an increasing sequence $\left(b_{m}\right)_{m}$ such that $\bigcup_{i=0}^{n} A+i=\Delta\left(\left(b_{m}\right)_{m}\right)$, where $\Delta\left(\left(b_{m}\right)_{m}\right)$ denote the set of differences of $\left(b_{m}\right)_{m}$ defined by $\Delta\left(\left(b_{m}\right)_{m}\right)=$ $\left\{b_{j}-b_{i}: 1 \leq i<j\right\}$. Pick $b_{j_{1}}, b_{j_{2}}\left(j_{1}<j_{2}\right)$ such that $\left|b_{j_{2}}-b_{j_{1}}\right|>n$, then it holds

$$
\left|b_{j_{2}}-b_{j_{1}}\right|=\left|\left(b_{j_{m}}-b_{j_{1}}\right)-\left(b_{j_{m}}-b_{j_{2}}\right)\right|, \quad \forall m \in \mathbb{N}
$$

which means that the distance $\left|b_{j_{2}}-b_{j_{1}}\right|$ between elements of $\cup_{i=o}^{n} A+i$ appears infinitely, which is not the case taking into account the way in which was defined $A$. Now, the same is true for any shifted $\Delta$-set $(\Delta+k)$ since obviously

$$
\left|b_{j_{2}}-b_{j_{1}}\right|=\left|\left(b_{j_{m}}-b_{j_{1}}+k\right)-\left(b_{j_{m}}-b_{j_{2}}+k\right)\right|, \quad \forall m \in \mathbb{N}, \forall k \in \mathbb{Z} .
$$


Then we conclude $\bigcup_{i=o}^{n} A+i \notin \Delta_{+}$. Recall that $\Delta$ can be written as a union of ultrafilters and the same is true for any shifted $\Delta$-set. Hence by 1.1.1), $\left(\bigcup_{i=0}^{n} A+i\right)^{c} \in\left(\Delta_{+}\right)^{*}$ which is equivalent to say

$$
\left(\bigcup_{i=0}^{n} A+i\right)^{c} \in\left(\Delta^{*}\right) \bullet
$$

\section{$2.2 \quad$ Return sets and density}

In the definition of the return sets $N_{T}(U, V)$, when $U$ is a singleton $\{x\}$, we write $N_{T}(x, V)$, which is nothing else than $N_{T}(x, V)=\left\{n \in \mathbb{N}: T^{n}(x) \in\right.$ $V\}$. The purpose of this section is to analyze which kind of properties of density can the sets $N(x, U)$ and $N(U, V)$ have for a given hypercyclic operator, and classify the hypercyclic operators accordingly to these properties. Throughout this section $X$ is assumed to be an $F$-space.

Proposition 34. There does not exist $x \in X$ such that $N(x, U)$ is syndetic, for every opene set $U \subset X$.

Proof. Assume, towards a contradiction, that there exists $x \in X$ such that $N(x, U)$ is syndetic, which is equivalent to say $\underline{B d}(N(x, U))>0$, all this for every opene set $U \subset X$.

Take $U, V$ opene sets such that $x \in U, 0 \in V$ and $U \cap V=\emptyset$. If we denote the maximum gap of $N(x, U)$ as $m$, then by continuity there exists $W$ a neighbourhood of zero such that $T^{j}(W) \subset V, j=0,1, \ldots, m$. Let $n$ such that $T^{n}(x) \in W$ and $T^{n+j} x \in V, j=0,1, \ldots, m+1$, therefore $\{n, n+1, \ldots, n+m+1\} \notin N(x, U)$ which is a contradiction since this implies that there are gaps in $N(x, U)$ with length greater than $m$. 


\section{2. $\mathscr{F}$-OPERATORS}

It is also true,

Proposition 35. There does not exist $x \in X$ such that $N(x, U)$ is thick, for every opene set $U \subset X$.

Proof. Assume, towards a contradiction, that there exists $x \in X$ such that $N(x, U)$ is thick, which is equivalent to say $\overline{B d}(N(x, U))=1$, all this for every opene set $U \subset X$. This implies that for every opene set $U$, there exists $n$ such that $n, n+1 \in N(x, U)$, then

$$
T(U) \cap U \neq \emptyset,
$$

for every opene set $U$. Let $T z=y$. Since $X$ is Hausdorff, then there exists $V_{y}, V_{z}$ open neighborhoods of $y$ and $z$ respectively, such that $V_{y} \cap V_{z}=\emptyset$. On the other hand, by continuity of $T$ there exists an open neighborhood of $z$ denoted $\bar{V}_{z}$, such that $T\left(\bar{V}_{z}\right) \subset V_{y}$. Set $\hat{V}_{z}=V_{z} \cap \bar{V}_{z} . \hat{V}_{z}$ is non-empty because $z \in \hat{V}_{z}$, then $T\left(\hat{V}_{z}\right) \subset V_{y}$ and hence $T\left(\hat{V}_{z}\right) \cap \hat{V}_{z}=\emptyset$, which is a contradiction with condition 2.2.1.

Now, we want enumerate all the properties appearing when $N(x, U)$ has density (any of the 4 possibilities illustrated in Definition 9 ) greater than 0 or equal to 1. By Proposition 34, Proposition 35 and condition 1.1.2 we have in fact only 3 possibilities, previously defined, then let us agree that

- $T$ is frequently hypercyclic if and only if $T$ has (P1)

- $T$ is $\mathfrak{U}$-frequently hypercyclic if and only if $T$ has (P2)

- $T$ is reiteratively hypercyclic if and only if $T$ has (P3).

Concerning $N(U, V)$ we have 8 further properties. 
Definition 36. Let $T \in \mathcal{L}(X)$ such that for every $U, V$ opene sets in $X$, we have

- $\underline{B d}(N(U, V))=1$. Then we say that $T$ has (P4)

- $\underline{d}(N(U, V))=1$. Then we say that $T$ has (P5)

- $\bar{d}(N(U, V))=1$. Then we say that $T$ has $(P 6)$

- $\overline{B d}(N(U, V))=1$. Then we say that $T$ has $(P 7)$

- $\underline{B d}(N(U, V))>0$. Then we say that $T$ has (P8)

- $\underline{d}(N(U, V))>0$. Then we say that $T$ has (P9)

- $\bar{d}(N(U, V))>0$. Then we say that $T$ has (P10)

- $\overline{B d}(N(U, V))>0$. Then we say that $T$ has (P11).

Please observe that syndetic operators are those satisfying property $(P 8)$ and weakly mixing those satisfying property $(P 7)$. By Proposition 22, operators having $(P 8)$ also have $(P 7)$.

Obviously we have the following chain of implications $(P 1) \longrightarrow(P 2) \longrightarrow$ (P3).

In general, $(P 3)$ implies $(P 8)$ for any $T \in \mathcal{L}(X)$ as the following proposition indicates.

Proposition 37. Let $T \in \mathcal{L}(X)$ a hypercyclic operator satisfying that for any $U$ opene set in $X$, there exists $x \in X$ such that $N(x, U)$ has positive upper Banach density. Then

$$
N(U, V) \in \bigcap_{t \in N(U, V)}\left(\Delta^{*}+t\right)
$$


for every $U, V$ opene sets in $X$. In particular, any reiteratively hypercyclic operator is syndetic, since the family of syndetic sets is shift invariant.

Please note that the family $\Delta^{*}$ is not shift invariant $\left(2 \mathbb{N} \in \Delta^{*}\right.$ but $\left.2 \mathbb{N}+1 \notin \Delta^{*}\right)$, hence Proposition 37 does not allow us to conclude that $T$ is necessarily a $\Delta^{*}$-operator.

Proof. Let $U, V$ opene sets in $X$. Pick, $n \in N(U, V)$, then $T^{n}(U) \cap V \neq$ $\emptyset$. Define the opene set $U_{n}:=U \cap T^{-n}(V)$. Let $x \in X$ such that $\overline{B d}\left(N\left(x, U_{n}\right)\right)>0$.

On the other hand, it is a well known fact that

$$
N\left(x, U_{n}\right)-N\left(x, U_{n}\right)+n \subseteq N(U, V) .
$$

Let $s_{1}, s_{2} \in N\left(x, U_{n}\right)$. Then, we have

$$
T^{s_{1}-s_{2}+n}\left(T^{s_{2}} x\right)=T^{n}\left(T^{s_{1}} x\right) \in V .
$$

and identity $(2.2 .2)$ holds. Hence, we conclude by proposition 1.1 (31).

In particular, in the frame of weighted backward shifts we have more information.

Proposition 38. If $B_{w}$ is reiteratively hypercyclic on $X=l_{p}(\mathbb{Z}),(1 \leq p<$ $\infty)$ or $c_{0}(\mathbb{Z})$, then it is $\Delta^{*}$-backward weighted shift on $X$.

First, we will need the following lemmas.

Lemma 39. Let $U, V$ opene sets in $X$ such that $U \cap V \neq \emptyset$, if $T$ is reiteratively hypercyclic on $X$ then $N(U, V) \in \Delta^{*}$.

Proof. It follows from proposition 37 . 
Let $\left(w_{k}\right)_{k \in \mathbb{Z}}$ be a bounded weight. Recall that $B(x ; r)=\{y \in X$ : $\|x-y\|<r\}$ and

$$
\begin{gathered}
A_{M, j}:=\left\{n \in \mathbb{N}: \prod_{i=j+1}^{j+n}\left|w_{i}\right|>M\right\} \\
\bar{A}_{M, j}=\left\{n \in \mathbb{N}: \frac{1}{\prod_{i=j-n+1}^{j}\left|w_{i}\right|}>M\right\} .
\end{gathered}
$$

Let $R>1, j \in \mathbb{Z}$. Let $\mathfrak{U}$ the set of opene sets in $X$. Consider the following subcollection of $\mathfrak{U}$ :

$$
U_{R, j}=\left\{U \in \mathfrak{U}:\left|e_{j}^{*}(x)\right|>\frac{1}{R}, \forall x \in U\right\} .
$$

Note that $U_{R, j} \neq \emptyset$. In fact, let $M>0$ such that $M R>1$, then $B((M+$ 1) $\left.e_{j} ; \frac{1}{M R}\right) \in U_{R, j}$.

Lemma 40. Let $M>0, j \in \mathbb{Z}$. Suppose there exists $U \in U_{R, j}$ such that for any opene subset $\widetilde{U}$ of $U$ it holds $N\left(\widetilde{U}, B\left((M+1) e_{j} ; \frac{1}{M R}\right)\right) \in \Delta^{*}$ for some $R>1$ such that $M R>1$, then $A_{M ; j} \in \Delta^{*}$ and $\bar{A}_{M ; j} \in \Delta^{*}$.

Proof. Let $(z(m))_{m}$ a dense set in $X$ such that $z(m)=\left(z(m)_{1}, \ldots, z(m)_{m}, 0 \ldots\right)$ and $U_{m}=B(z(m) ; 1 / m)$. Let $U \in U_{R, j}$ such that for any opene subset $\widetilde{U}$ of $U$ we have $N\left(\widetilde{U}, B\left((M+1) e_{j} ; \frac{1}{M R}\right)\right) \in \Delta^{*}$ for some $R>1$ such that $M R>1$, then there exists $m$ such that $U_{m} \subset U$ and hence $N\left(U_{m}, B\left((M+1) e_{j} ; \frac{1}{M R}\right)\right) \in \Delta^{*}$. Pick $r \in N\left(U_{m}, B\left((M+1) e_{j} ; \frac{1}{M R}\right)\right)$ with $r>m$ and $x \in U_{m}$ such that $B_{w}^{r} x=y \in B\left((M+1) e_{j} ; \frac{1}{M R}\right)$.

Then,

$$
\left|\prod_{i=j+1}^{j+r} w_{i} x_{j+r}-(M+1)\right|<\frac{1}{M R} .
$$




$$
\left|\prod_{i=t+1}^{t+r} w_{i} x_{t+r}\right|<\frac{1}{M R}, \quad \forall t \neq j
$$

By 2.2.3 we get,

$$
\left|\prod_{i=1}^{r} w_{i+j}\right|>\left|\prod_{i=1}^{r} w_{i+j} x_{r+j}\right|>M,
$$

where the first inequality follows since $r>m$. We conclude that $N\left(U_{m}, B((M+\right.$ 1) $\left.\left.e_{j} ; \frac{1}{M R}\right)\right) \backslash\{1 \ldots m\} \subseteq A_{M ; j}$, then $A_{M ; j} \in \Delta^{*}$.

On the other hand, by 2.2.4 we get $\prod_{i=j-r+1}^{j}\left|w_{i} x_{j}\right|<\frac{1}{M R}$, hence

$$
\prod_{i=j-r+1}^{j}\left|w_{i}\right| \frac{1}{R}<\prod_{i=j-r+1}^{j}\left|w_{i} x_{j}\right|<\frac{1}{M R}
$$

Then, $\prod_{i=j-r+1}^{j}\left|w_{i}\right|<\frac{1}{M}$ and $N\left(U_{m}, B\left((M+1) e_{j} ; \frac{1}{M R}\right)\right) \subseteq \bar{A}_{M ; j}$, hence $\bar{A}_{M ; j} \in \Delta^{*}$.

\section{Proof of proposition 38}

Proof. Suppose $B_{w}$ is not a $\Delta^{*}$-backward weighted shift on $X$, then by proposition 27, there exists $M>0$ and $j \in \mathbb{Z}$ such that $A_{M ; j} \notin \Delta^{*}$ or $\bar{A}_{M ; j} \notin \Delta^{*}$. Let $R>1$ such that $M R>1$. Hence by lemma 40

$$
\forall U \in U_{R, j} \quad \exists \widetilde{U} \subseteq U: N\left(\widetilde{U}, B\left((M+1) e_{j} ; \frac{1}{M R}\right)\right) \notin \Delta^{*} .
$$

Note that $B\left((M+1) e_{j} ; \frac{1}{M R}\right) \in U_{R, j}$, then take $U=B\left((M+1) e_{j} ; \frac{1}{M R}\right)$, we have there exists $\widetilde{U} \subseteq U$ such that $N(\widetilde{U}, U) \notin \Delta^{*}$, which by lemma $39, B_{w}$ can not be reiteratively hypercyclic. 
Analogously, we have the unilateral version following the same sketch of proof.

Proposition 41. If $B_{w}$ is reiteratively hypercyclic on $X=l_{p}\left(\mathbb{Z}_{+}\right),(1 \leq$ $p<\infty)$ or $c_{0}\left(\mathbb{Z}_{+}\right)$, then it is $\Delta^{*}$-backward weighted shift on $X$.

Conversely, we obtain that $P 8$ does not imply $P 3$. In fact,

Proposition 42. There exists an $\mathrm{JP}^{*}$-weighted backward shift which is not reiteratively hypercyclic on $c_{0}\left(\mathbb{Z}_{+}\right)$and $l_{p}\left(\mathbb{Z}_{+}\right)(1 \leq p<\infty)$. In particular, $P 8$ does not imply P3.

Proof. It follows by proposition 32 and proposition 41 .

Now, let us study the relationship between the rest of properties introduced in definition 36 .

Proposition 43. Let $X=c_{0}\left(\mathbb{Z}_{+}\right)$, then

i) (P7) does not imply (P10). Consequently, $(P 7)$ does not imply (P6) and (P11) does not imply (P10)

ii) (P6) does not imply (P9). Consequently, (P6) does not imply (P5) and (P10) does not imply (P9)

iii) (P5) does not imply (P8). Consequently, (P5) does not imply (P4) and (P9) does not imply (P8).

Proof. i) Consider the weight

$$
w=(\underbrace{1, \ldots, 1}_{m_{1}}, 2,2^{-1}, \underbrace{1, \ldots, 1}_{m_{2}}, 2,2,2^{-2}, \underbrace{1, \ldots, 1}_{m_{3}}, 2,2,2,2^{-3}, \underbrace{1, \ldots, 1}_{m_{4}}, \ldots)
$$

with $\left(m_{k}\right)_{k \in \mathbb{N}}$ suitably chosen such that $\bar{d}\left(N\left(B(0 ; 1), B\left(2 e_{0} ; 1 / 2\right)\right)\right)=0$, where $\left(e_{n}\right)_{n=0}^{\infty}$ is the canonical basis in $c_{0}\left(\mathbb{Z}_{+}\right)$and $B(x ; r)$ denote the ball 
of radius $\mathrm{r}$ and centered at $x$. Let $x \in B(0 ; 1)$, then $\left|x_{t}\right|<1$ for every $t \in \mathbb{N}$. On the other hand,

$$
B_{w}^{n} x=\left(\prod_{i=0}^{n} w_{i} x_{n}, \prod_{i=0}^{n} w_{i+1} x_{n+1}, \cdots\right)
$$

and $\left|\prod_{i=0}^{n} w_{i} x_{n}-2\right|>1$ whenever $\prod_{i=0}^{n} w_{i}=1$. Hence,

$$
\left\{n \in \mathbb{N}: \prod_{i=0}^{n} w_{i}=1\right\} \subseteq N\left(B(0 ; 1), B\left(2 e_{0} ; 1 / 2\right)\right)^{c} .
$$

Then $\left(m_{k}\right)_{k \in \mathbb{N}}$ must be chosen such that $\underline{d}\left(\left\{n: \prod_{i=0}^{n} w_{i}=1\right\}\right)=1$, which implies $\underline{d}\left(N\left(B(0 ; 1), B\left(2 e_{0} ; 1 / 2\right)\right)^{c}\right)=1$ and consequently

$$
\bar{d}\left(N\left(B(0 ; 1), B\left(2 e_{0} ; 1 / 2\right)\right)\right)=0
$$

by condition 1.1 .3$)$, i.e., $B_{w}$ has not $(P 10)$.

Observe that $\sup _{n} \prod_{i=0}^{n} w_{i}=\infty$, hence $B_{w}$ is weakly mixing, i.e., has $(P 7)$.

ii) Consider the weight,

$$
w=(\underbrace{1, \cdots, 1}_{m_{1}}, \underbrace{2, \cdots, 2}_{n_{1}}, 2^{-n_{1}}, \underbrace{1, \cdots, 1}_{m_{2}}, \underbrace{2, \cdots, 2}_{n_{2}}, 2^{-n_{2}}, \underbrace{1, \cdots, 1}_{m_{3}}, \underbrace{2, \cdots, 2}_{n_{3}}, 2^{-n_{3}}, \cdots) .
$$

Recall that $\overline{\mathcal{D}}$ is a shift invariant family, then arguing as in $i$ ) and applying proposition 28, the sequences $\left(m_{k}\right)_{k},\left(n_{k}\right)_{k}$ must be chosen in such a way that the following hold

- $\bar{d}\left(\left\{n: \prod_{i=0}^{n} w_{i}=1\right\}\right)=1$

- $\bar{d}\left(A_{M}\right)=\bar{d}\left(\left\{n: \prod_{i=0}^{n} w_{i}>M\right\}\right)=1$, for every $M>0$. 
Define sequences of intervals in the following way,

$$
A_{k}=\left[10^{2^{2 k}}, 10^{2^{2 k+1}}\right], \quad B_{k}=\left[10^{2^{2 k+1}}, 10^{2^{2(k+1)}}\right], \quad k \in \mathbb{N} .
$$

It can be verified that $A=\cup_{k \in \mathbb{N}} A_{k}$ and $B=\cup_{k \in \mathbb{N}} B_{k}$ are disjoint with $\bar{d}(A)=\bar{d}(B)=1$. Hence, setting $m_{k}=\left|A_{k}\right|, n_{k}=\left|B_{k}\right|$ for every $k$, we are done.

iii) Let $m_{k}=10^{2^{k}}, k \in \mathbb{N}$ and consider the weight

$$
w=(1,2,2^{-1}, 1,1, \underbrace{2, \cdots, 2}_{m_{1}}, 2^{-m_{1}}, 1,1,1, \underbrace{2, \cdots, 2}_{m_{2}}, 2^{-m_{2}}, 1,1,1,1, \underbrace{2, \cdots, 2}_{m_{3}}, 2^{-m_{3}}, \cdots) .
$$

The set $A_{1}=\left\{n: \prod_{i=0}^{n} w_{i}>1\right\}$ is not syndetic, hence $B_{w}$ has not (P8) by proposition 28. On the other hand,

$$
\underline{d}\left(A_{M}\right)=\underline{d}\left(\left\{n: \prod_{i=0}^{n} w_{i}>M\right\}\right)=1,
$$

for every $M>0$, hence $B_{w}$ has $(P 5)$, again by proposition 28 since $\underline{\mathcal{D}}$ is a shift invariant family.

Mixing operators obviously have $(P 4)$, but the converse is false, this is the argument of the next proposition.

Proposition 44. There exists a non-mixing operator on $c_{0}\left(\mathbb{Z}_{+}\right)$satisfying property $(P 4)$.

Proof. Let $m_{k}=10^{2^{k}}, k \in \mathbb{N}$ and consider the weight $w=\left(w_{n}\right)_{n=0}^{\infty}$ defined as

$$
w=(1, \underbrace{2, \ldots, 2}_{m_{1}}, 2^{-m_{1}}, \underbrace{2, \ldots, 2}_{m_{2}}, 2^{-m_{2}}, \underbrace{2, \ldots, 2}_{m_{3}}, 2^{-m_{3}}, \ldots) .
$$

$B_{w}$ is not mixing since is not satisfied $\lim _{n} \prod_{i=0}^{n} w_{i}=\infty$. On the other hand, $\underline{B d}\left(A_{M}\right)=1$ for every $M>0$. In fact, let $M>0$, then there exists $n \in \mathbb{N}$ such that $2^{n-1} \leq M<2^{n}$. 
Let $s>n+1$, set

$$
\alpha_{s}:=\liminf _{k \rightarrow \infty}\left|A_{M} \cap[k, k+s]\right|=s-n .
$$

Hence,

$$
\underline{B d}\left(A_{M}\right)=\lim _{s \rightarrow \infty} \frac{\alpha_{s}}{s}=\lim _{s \rightarrow \infty} \frac{s-n}{s}=1,
$$

and we conclude by proposition 28 , since $\underline{\mathcal{B D}}$ is a shift invariant family.

Proposition 45. Property $(P 8)$ does not imply $(P 6)$ in $l_{1}\left(\mathbb{Z}_{+}\right)$. Consequently,

- (P8) does imply neither (P4) nor (P5)

- (P9) does not imply (P5)

- neither (P9) nor (P10) imply (P6).

Proof. Consider $A_{n}=[\underbrace{2, \ldots, 2}_{n-\text { times }}, 2^{-n}], B_{1}=A_{1}, B_{n}=\left[B_{n-1}, A_{n}, B_{n-1}\right]$. Consider the weight

$$
w=(\underbrace{\underbrace{A_{1}, A_{2}, A_{1}}_{1}, A_{3}}, \underbrace{A_{1}, A_{2}, A_{1}}, A_{4}, \underbrace{\underbrace{A_{1}, A_{2}, A_{1}}}, A_{3}, \underbrace{A_{1}, A_{2}, A_{1}}, \ldots) .
$$

By proposition 28, $B_{w}$ is syndetic, i.e. has property $(P 8)$. Let us prove that

$$
\bar{d}\left(N\left(B(0 ; 1), B\left(2 e_{0} ; 1 / 2\right)\right)\right)=2 / 3<1 .
$$

Firstly, let us see that

$$
\left\{n \in \mathbb{N}: \prod_{i=0}^{n} w_{i}=1\right\} \subseteq\left[N\left(B(0 ; 1), B\left(2 e_{0} ; 1 / 2\right)\right)\right]^{c} .
$$


Let $n \in \mathbb{N}$ such that $\prod_{i=0}^{n} w_{i}=1$ and $x \in B(0 ; 1)$, then $\left|x_{t}\right|<1$ for every $t \in \mathbb{N}$. On the other hand,

$$
\left\|B_{w}^{n} x-2 e_{0}\right\|=\left|\prod_{i=0}^{n} w_{i} x_{n}-2\right|+\sum_{k=1}^{\infty}\left|\prod_{i=0}^{n} w_{i+k} x_{n+k}\right| \geq\left|\prod_{i=0}^{n} w_{i} x_{n}-2\right|>1
$$

Hence, $B_{w}^{n} x \notin B\left(2 e_{0} ; 1 / 2\right)$ and 2.2 .5 holds.

Now, $\left|B_{n}\right|=3 \cdot 2^{n}-n-3$ and $\alpha_{n}=\left|\left\{k \leq\left|B_{n}\right|: \prod_{i=0}^{k} w_{i}=1\right\}\right|=2^{n}-1$.

By 2.2.5 we have,

$$
\begin{gathered}
\bar{d}\left(N\left(B(0 ; 1), B\left(2 e_{0} ; 1 / 2\right)\right)\right) \leq \bar{d}\left(\left\{k \in \mathbb{N}: \prod_{i=0}^{k} w_{i}>1\right\}\right) \\
=\underset{n}{\lim \sup } \frac{\left|\left\{k \in[1, n]: \prod_{i=0}^{k} w_{i}>1\right\}\right|}{n}=\limsup _{n} \frac{\left|\left\{k \leq\left|B_{n}\right|+\left|A_{n+1}\right|: \prod_{i=0}^{k} w_{i}>1\right\}\right|}{\left|B_{n}\right|+\left|A_{n+1}\right|} \\
=\lim _{n} \frac{\left|B_{n}\right|-\alpha_{n}+n+1}{\left|B_{n}\right|+n+2}=\lim _{n} \frac{2 \cdot 2^{n}-1}{3 \cdot 2^{n}-1}=2 / 3 .
\end{gathered}
$$

The following diagram shows the main results discussed above. Dashed arrows fail to hold. 


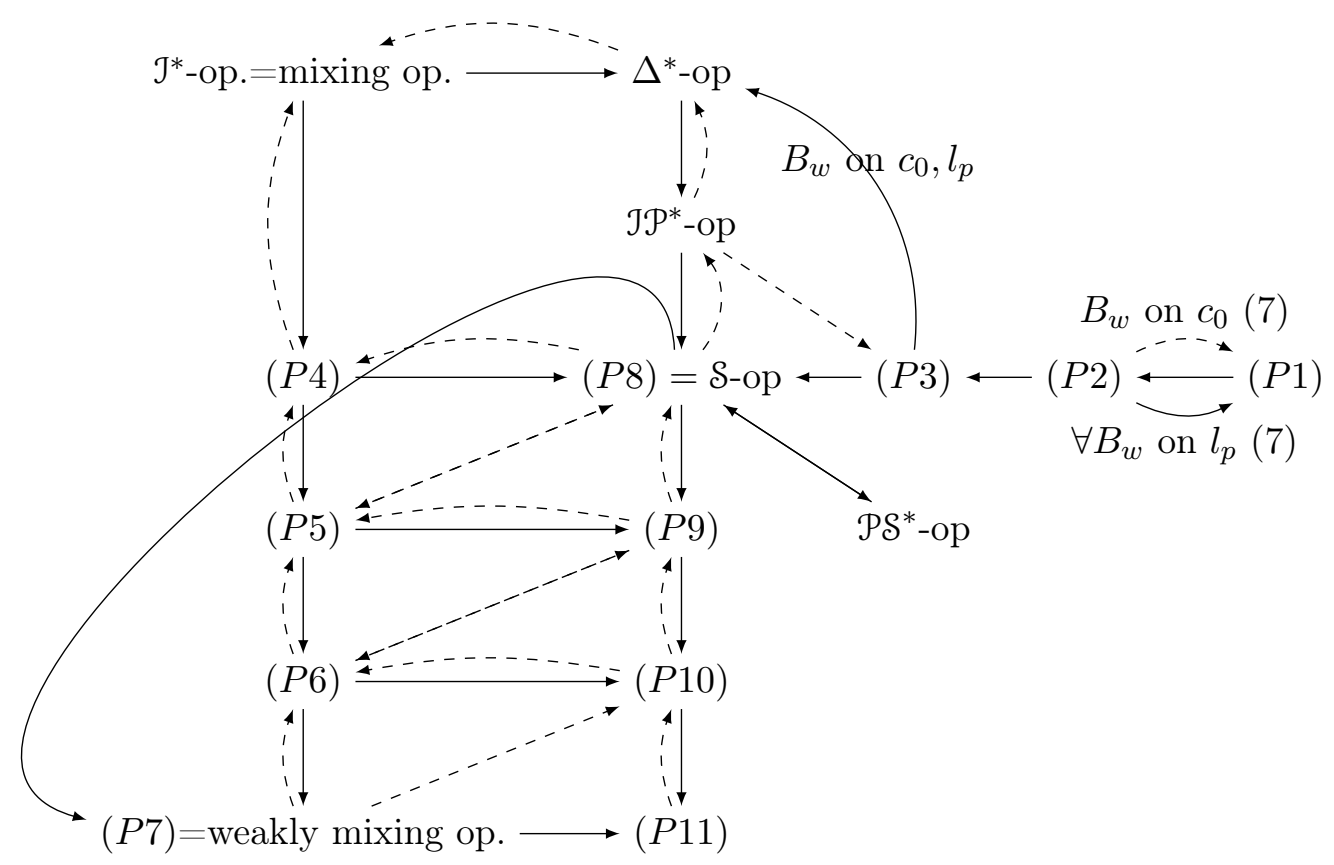

Figure 2

\subsection{Questions}

In proposition 38, we have seen that a reiteratively hypercyclic weighted backward shift on $c_{0}$ or $l_{p}(1 \leq p<\infty)$ is $\Delta^{*}$-operator. One could wonder whether this holds beyond the weighted backward shift setting,

Question 46. Must a reiteratively hypercyclic operator $T \in \mathcal{L}(X)$ be a $\Delta^{*}$-operator?

Please note that a $\Delta^{*}$-operator $T$, satisfies $\Delta^{*}-\lim _{n}\left\|T^{n}\right\|=\infty$. In fact, 
let $M>0$ and set $V=\{x \in X:\|x\|>M\}$, then

$$
\left\{n \in \mathbb{N}: T^{n}(B(0,1)) \cap V \neq \emptyset\right\} \subseteq\left\{n \in \mathbb{N}:\left\|T^{n}\right\|>M\right\} .
$$

Hence in order to exhibit a reiteratively hypercyclic but not $\Delta^{*}$-operator, arguing as in (1) and applying (1.1.1), it suffices to show the existence of a real positive $M$ such that

$$
\left(n_{k}\right)_{k}=\left\{n \in \mathbb{N}:\left\|T^{n}\right\| \leq M\right\} \in \Delta
$$

and

$$
\sum_{k=1}^{\infty}\left(\frac{n_{k}}{n_{k+1}}\right)^{2}<\infty .
$$

But condition 2.3.1 and 2.3.2 can not be satisfied at the same time. In fact, assume 2.3.1, hence there exists a distance $a \in \mathbb{N}$ that appears infinitely many times between elements of $\left(n_{k}\right)_{k}$, which implies there exists $\bar{a} \leq a$ and a subsequence $\left(n_{k_{j}}\right)_{j}$ such that $n_{k_{j}+1}-n_{k_{j}}=\bar{a}$ for every $j \in$ $\mathbb{N}$, then $\lim _{j} n_{k_{j}} / n_{k_{j}+1}=1$. Consequently, 2.3.2 can not hold and this attempt of answering question 46 fails.

Finally, we were not able to answer the following:

Question 47. Does there exist $T \in \mathcal{L}(X)$ satisfying (P9) but not $(P 7)$ ? In other words, does there exist $T \in \mathcal{L}(X)$ being $\underline{\mathcal{D}}$-operator but not weakly mixing?

Note that if it were the case, then such operator $T$ must not be weighted shift. Furthermore, we underline that in the search for such operator, [ (6), Theorem 1.2] could be of great help. 
2. $\mathscr{F}$-OPERATORS 


\section{Recurrence properties defined via essential idempotents of $\beta \mathbb{N}$}

Let us introduce the following notion:

Definition 48. The sequence of operators $\left(T_{n}\right)_{n}$ satisfies property $\mathcal{P}_{\mathscr{F}}$ if for any $U$ opene set in $X$ there exists $x \in X$ such that

$$
N(x, U)=\left\{n \in \mathbb{N}: T_{n} x \in U\right\} \in \mathscr{F} .
$$

An operator $T$ satisfies property $\mathcal{P}_{\mathscr{F}}$ if the sequence $\left(T^{n}\right)_{n}$ satisfies property $\mathcal{P}_{\mathscr{F}}$ as well.

The aim of this chapter is to generalize the main result of a recent paper of Costakis and Parissis (19), using a strong result of Bergelson and Mccutcheon (10) in the vein of Szemerédi's theorem, leading us to a characterization of those operators satisfying property $\mathcal{P}_{\overline{\mathcal{B D}}}$.

In terms of recurrence, in (19) Costakis and Parissis are mainly concerned with topologically multiply recurrence. 


\section{RECURRENCE PROPERTIES DEFINED VIA ESSENTIAL IDEMPOTENTS OF $\beta \mathbb{N}$}

Definition 49. An operator $T \in \mathcal{L}(X)$ is topologically multiply recurrent if for every opene set $U$ in $X$ and every $r \in \mathbb{N}$, there is some $k \in \mathbb{N}$ such that

$$
U \cap T^{-k} U \cap \ldots \cap T^{-r k} U \neq \emptyset .
$$

Now, the main result of (19):

Theorem 50. (19)

Let $\left(\lambda_{n}\right)_{n \in \mathbb{N}}$ be a sequence of non-zero complex numbers which satisfies

$$
\lim _{n \rightarrow \infty} \frac{\left|\lambda_{n}\right|}{\left|\lambda_{n+\tau}\right|}=1
$$

for some positive integer $\tau$. Let $T \in \mathcal{L}(X)$ such that the family $\left(\lambda_{n} T^{n}\right)_{n}$ satisfies property $\mathcal{P}_{\overline{\mathcal{D}}}$. Then $T$ is topologically multiply recurrent.

More on recurrence and hypercyclicity can be found in (18).

We would like to generalize the result of Costakis and Parissis by showing a stronger kind of recurrence. In (3.0.1) we will be asking that the intersection is not only non-empty but satisfies some condition involving Banach density. On the other hand, the mere existence of some $k \in \mathbb{N}$ will not be enough for us, instead we want that the set of such $k$ be infinite and with very specific algebraic properties.

We will be mainly concerned with the so-called essential idempotents on $\beta \mathbb{N}$, defined as

$$
\mathcal{E}=\{p \in E(\mathbb{N}): \overline{B d}(A)>0, \forall A \in p\}
$$

A set $A \subseteq \mathbb{N}$ is called an $\mathcal{E}$-set if there exists $p \in \mathcal{E}$ such that $A \in p$. Now, we will introduce what we call $\mathcal{E}$-recurrence for a linear operator. Let $m \in \mathbb{N}$. 
Definition 51. $T \in \mathcal{L}(X)$ is topologically $(\mathcal{E}, m)$-recurrent with respect to $\lambda=\left(\lambda_{n}\right)_{n}$ if there exists some $p \in \mathcal{E}$ such that for any $U_{1}, \ldots, U_{m}$ opene sets in $X$, there exists $x_{1}, \ldots, x_{m} \in X$, such that for any $\left(r_{1}, \ldots, r_{m}\right) \in \mathbb{N}^{m}$, we have

$$
\bigcap_{i=1}^{m}\left\{k \in \mathbb{N}: \overline{B d}\left(a \in \mathbb{N}: \lambda_{a} T^{a} x_{i} \in \cap_{j=0}^{r_{i}} T^{-j k}\left(U_{i}\right)\right)>0\right\} \in p .
$$

Let us say $T$ is topologically $\mathcal{E}$-recurrent with respect to $\left(\lambda_{n}\right)_{n}$ when $T$ is topologically $(\mathcal{E}, 1)$-recurrent with respect to $\left(\lambda_{n}\right)_{n}$. In the case, $\left(\lambda_{n}\right)_{n}=1$, we simply say $T$ topologically $\mathcal{E}$-recurrent.

We state the main result of this chapter:

Theorem 52. Let $\left(\lambda_{n}\right)_{n}$ be a sequence of non-zero complex numbers and let $p \in \mathcal{E}$ such that there exists $A \in p$ for which

$$
\overline{\mathcal{B D}}^{*}-\lim _{n}\left|\frac{\lambda_{n}}{\lambda_{n+k}}\right|=1, \forall k \in A .
$$

Then the family $\left(\lambda_{n} T^{n}\right)_{n}$ acting on $X$ satisfies property $\mathcal{P}_{\overline{\mathcal{B D}}}$ if and only if $T$ is topologically $(\mathcal{E}, m)$-recurrent with respect to $\left(\lambda_{n}\right)_{n}$, for any $m \in \mathbb{N}$.

Proof. $(\Leftarrow)$ Evident, this holds without the condition on $\lambda=\left(\lambda_{n}\right)_{n}$.

$(\Rightarrow)$ Let $\left(\lambda_{n}\right)_{n}$ a sequence of non-zero complex numbers as in the statement of the theorem, then the family $\left(\lambda_{n} T^{n}\right)_{n}$ has the property $\mathcal{P}_{\overline{\mathcal{B D}}}$ if and only if $\left(\left|\lambda_{n}\right| T^{n}\right)_{n}$ has the property $\mathcal{P}_{\overline{\mathcal{B D}}}$, and the proof follows the same lines as Lemma $3.7(19)$, replacing $\overline{B d}$ instead of $\bar{d}$. So, we may assume that $\left(\lambda_{n}\right)_{n}$ is a sequence of positive numbers such that for some $p \in \mathcal{E}$ and some $A \in p$, we have $\lim _{n} \lambda_{n} / \lambda_{n+k}=1$ for every $k \in A$.

First, let us prove the case $m=1$. So, let $U$ opene set in $X$ and $r \in \mathbb{N}$. Then there exists $y \in U$ and a positive number $\epsilon$ such that $B(y, \epsilon) \subseteq U$. 


\section{RECURRENCE PROPERTIES DEFINED VIA ESSENTIAL IDEMPOTENTS OF $\beta \mathbb{N}$}

Hence by the property $\mathcal{P}_{\overline{\mathcal{B D}}}$ of the family $\left(\lambda_{n} T^{n}\right)_{n}$, there exists $x \in X$ such that

$$
F=\left\{n \in \mathbb{N}: \lambda_{n} T^{n} x \in B(y, \epsilon / 2)\right\}
$$

has positive upper Banach density. Consider the family of polynomials $g_{1}(k)=-k, \ldots, g_{r}(k)=-r k$, hence $g_{1}, \ldots g_{r} \in \mathcal{G}_{a}$ the group of admissible generalized polynomials, see page 10 (10) for the definition. Now by Theorem 1.25 (10) we have that

$$
W:=\{k \in \mathbb{N}: \overline{B d}(F \cap(F-k) \cap \ldots \cap(F-r k))>0\} \in \mathcal{E}^{*} .
$$

Hence, $W \in q, \forall q \in \mathcal{E}$.

On the other hand, recall that 3.0 .2 holds for some $p \in \mathcal{E}$ and some $A \in p$, hence,

$$
W \cap A \in p .
$$

Fix $k \in W \cap A$. Denote

$$
M_{k, r}:=F \cap(F-k) \cap \ldots \cap(F-r k)=\{a: a, a+k, \ldots, a+r k \in F\} .
$$

As in the proof of Theorem 50 , for each $a \in M_{k, r}$ and $j=1 \ldots r$ we let

$$
\begin{gathered}
u:=\lambda_{a} T^{a} x \in B(y, \epsilon / 2) \\
u_{j}:=\lambda_{a+j k} T^{a+j k} x=\frac{\lambda_{a+j k}}{\lambda_{a}} T^{j k} u \in B(y, \epsilon / 2) .
\end{gathered}
$$

Let $M>0$ such that $\left\|u_{j}\right\| \leq M$ for any $j=1 \ldots r$.

On the other hand,

$$
\left\|T^{j k} u-u_{j}\right\|=\left\|\frac{\lambda_{a}}{\lambda_{a+j k}} u_{j}-u_{j}\right\|=\left|\frac{\lambda_{a}}{\lambda_{a+j k}}-1\right|\left\|u_{j}\right\|
$$

Now, set

$$
I_{j, k}=\left\{a \in \mathbb{N}:\left|\frac{\lambda_{a}}{\lambda_{a+j k}}-1\right|<\epsilon / 2 M\right\}
$$


Hence,

$$
I_{j, k} \in \overline{\mathcal{B D}}^{*},
$$

for every $j=1 \ldots r$, because by hypothesis $k \in W \cap A$ implies

$$
\overline{\mathcal{B D}}^{*}-\lim _{n} \frac{\lambda_{n}}{\lambda_{n+k}}=1
$$

and this in turn implies

$$
\overline{\mathcal{B D}}^{*}-\lim _{n} \frac{\lambda_{n}}{\lambda_{n+j k}}=1,
$$

for any $j=1 \ldots r$. We will prove this last implication later.

The family $\overline{\mathcal{B D}}^{*}$ is a filter. In fact, it can be written as an intersection of ultrafilters, as follows:

$$
\overline{\mathcal{B D}}^{*}=\bigcap_{p \in \mathscr{D}} p^{*}=\bigcap_{p \in \mathscr{D}} p=\{A \subseteq \mathbb{N}: A \in p, \forall p \in \mathscr{D}\},
$$

where $\mathscr{D}=\{p \in \beta \mathbb{N}: \overline{B d}(A)>0, \forall A \in p\}$. First, recall that $\mathscr{F}=\mathscr{F}^{*}$ when $\mathscr{F}$ is an ultrafilter. Indeed, $\mathscr{F} \subseteq \mathscr{F}^{*}$ follows from the fact that $\mathscr{F}$ is also a filter. Conversely, if $A \in \mathscr{F}^{*}$, then $A^{c} \in \mathscr{F}$ by Theorem 3.6 (27), hence $A \cap A^{c} \neq \emptyset$ which is a contradiction. Second, obviously

$$
\bigcup_{p \in \mathscr{D}} p=\{A \subseteq \mathbb{N}: A \in p, p \in \mathscr{D}\} \subseteq \overline{\mathcal{B D}} .
$$

Conversely, Let $A \in \overline{\mathcal{B D}}$, then by Lemma 2.3 (28) there exists $p \in \mathscr{D}$ such that $A \in p$, then $\overline{\mathcal{B D}}=\bigcup_{p \in \mathscr{D}} p$ and consequently $\overline{\mathcal{B D}}^{*}=\bigcap_{p \in \mathscr{D}} p^{*}$.

Hence by 3.0.6, we have that $I_{k}:=\cap_{j=1}^{r} I_{j, k} \in \overline{\mathcal{B D}}^{*}=\mathscr{D}^{*}$, i.e.

$$
I_{k} \in q, \forall q \in \mathscr{D} \text {. }
$$

Now, $\overline{B d}\left(M_{k, r}\right)>0$ by 3.0 .3 . Hence by Lemma $2.3(28)$ there exists $\widetilde{p} \in \mathscr{D}$ such that

$$
M_{k, r} \in \widetilde{p}
$$


By (3.0.7) and (3.0.8) we have $A_{k, r}:=I_{k} \cap M_{k, r} \in \widetilde{p}$. Hence,

$$
\overline{B d}\left(A_{k, r}\right)>0
$$

and

$$
A_{k, r} \subseteq\left\{a \in \mathbb{N}:\left\|T^{j k} u-u_{j}\right\|<\epsilon / 2,\left\|u_{j}-y\right\|<\epsilon / 2, j=1 \ldots r\right\},
$$

then

$$
A_{k, r} \subseteq\left\{a \in \mathbb{N}:\left\|T^{j k} u-y\right\|<\epsilon, j=1 \ldots r\right\} .
$$

Hence by (3.0.5) and 3.0.10 we obtain

$$
u, T^{k}(u), \ldots, T^{r k}(u) \in U,
$$

for every $a \in A_{k, r}$.

Now, by (3.0.4) and 3.0 .9 we have,

$$
\left\{k \in \mathbb{N}: \overline{B d}\left(a \in \mathbb{N}: \lambda_{a} T^{a} x \in \cap_{j=0}^{r} T^{-j k}(U)\right)>0\right\} \in p
$$

and $T$ is topologically $(\mathcal{E}, 1)$-recurrent with respect to $\lambda=\left(\lambda_{n}\right)_{n}$. To finish, with the case $m=1$, it remains to prove $\overline{\mathcal{B D}}^{*}-\lim _{n} \frac{\lambda_{n}}{\lambda_{n+k}}=1$ implies $\overline{\mathcal{B D}}^{*}-\lim _{n} \frac{\lambda_{n}}{\lambda_{n+j k}}=1$, for any $j=1 \ldots r$. In fact,

$$
\lambda_{a} / \lambda_{a+j k}=\lambda_{a} / \lambda_{a+k} \cdot \lambda_{a+k} / \lambda_{a+2 k} \cdot \ldots \cdot \lambda_{a+(j-1) k} / \lambda_{a+j k} .
$$

Now, let $V$ an open neighbourhood of 1 , then one can find $V_{1}, V_{2}, \ldots, V_{j}$ neighbourhoods of 1 such that

$$
\begin{aligned}
& \left\{n \in \mathbb{N}: \frac{\lambda_{n}}{\lambda_{n+k}} \in V_{1}\right\} \cap\left\{n \in \mathbb{N}: \frac{\lambda_{n+k}}{\lambda_{n+2 k}} \in V_{2}\right\} \cap \ldots \cap\left\{n \in \mathbb{N}: \frac{\lambda_{n+(j-1) k}}{\lambda_{n+j k}} \in V_{j}\right\} \subseteq \\
& \subseteq\left\{n \in \mathbb{N}: \frac{\lambda_{n}}{\lambda_{n+k}} \cdot \frac{\lambda_{n+k}}{\lambda_{n+2 k}} \cdot \ldots \cdot \frac{\lambda_{n+(j-1) k}}{\lambda_{n+j k}} \in V\right\}=\left\{n \in \mathbb{N}: \frac{\lambda_{n}}{\lambda_{n+j k}} \in V\right\} .
\end{aligned}
$$


By hypothesis,

$$
\left\{n \in \mathbb{N}: \lambda_{n+(t-1) k} / \lambda_{n+t k} \in V_{t}\right\} \in \overline{\mathcal{B D}}^{*},
$$

for $t=1 \ldots j$. Hence because $\overline{\mathcal{B D}}^{*}$ is a filter, we have

$$
\bigcap_{t=1}^{j}\left\{n \in \mathbb{N}: \frac{\lambda_{n+(t-1) k}}{\lambda_{n+t k}} \in V_{t}\right\} \in \overline{\mathcal{B D}}^{*} .
$$

Consequently, $\left\{n \in \mathbb{N}: \lambda_{n} / \lambda_{n+j k} \in V\right\} \in \overline{\mathcal{B D}}^{*}$. Finally, since $V$ was arbitrary, we conclude that

$$
\left\{n \in \mathbb{N}: \lambda_{n} / \lambda_{n+j k} \in V\right\} \in \overline{\mathcal{B D}}^{*},
$$

for every open neighbourhood $V$ of 1 and any $j=1 \ldots r$.

Let us finish the proof considering the case $m>1$. Let $\left(U_{i}\right)_{i=1}^{m}$ a finite collection of opene sets in $X$. For each $U_{i}$ we proceed as in the case $m=1$, then there exists $x_{i} \in X$ such that for any $r_{i} \in \mathbb{N}$ we have $W_{i} \in \mathcal{E}^{*}$ by 3.0.3. On the other hand, recall that $\mathcal{E}^{*}$ is a filter since, $\mathcal{E}^{*}=\cap_{p \in \mathcal{E}} p$ and intersection of ultrafilters is a filter, hence

$$
\cap_{1 \leq i \leq m} W_{i} \in \mathcal{E}^{*}
$$

and we can conclude $T$ is topologically $(\mathcal{E}, m)$-recurrent with respect to $\left(\lambda_{n}\right)_{n}$, for any $m \in \mathbb{N}$.

\subsection{Adjoints of multiplication operators}

An easy application of Theorem 52 can be seen in the setting of adjoints of multiplication operators (see (24), (4)) for an introduction. 


\section{RECURRENCE PROPERTIES DEFINED VIA ESSENTIAL IDEMPOTENTS OF $\beta \mathbb{N}$}

Fix a non-empty open connected set $\Omega \subset \mathbb{C}^{n}, n \in \mathbb{N}$, and $H$ a Hilbert space of holomorphic functions such that $H \neq\{0\}$ and for every $z \in \Omega$, the point evaluation functionals $f \mapsto f(z), f \in H$, are bounded.

Recall that every complex valued function $\phi: \Omega \rightarrow \mathbb{C}$ such that the pointwise product $\phi f \in H$, for every $f \in H$ is called a multiplier of $H$, and defines a multiplication operator $M_{\phi}: H \rightarrow H$ defined as

$$
M_{\phi}(f)=\phi f, \quad f \in H .
$$

Recall that an operator $T$ is called recurrent if $N(U, U) \neq \emptyset$ for every opene set $U$ in $X$. The following is an improvement of proposition 6.1 (19).

Corollary 53. Suppose that every non-constant bounded holomorphic function $\phi$ on $\Omega$ is a multiplier of $H$ such that $\left\|M_{\phi}\right\|=\|\phi\|_{\infty}$. Then for each such $\phi$ the following are equivalent.

i) $M_{\phi}^{*}$ is topologically $\mathcal{E}$-recurrent

ii) $M_{\phi}^{*}$ is recurrent

iii) $M_{\phi}^{*}$ is frequently hypercyclic

iv) $M_{\phi}^{*}$ is hypercyclic

v) $\phi(\Omega) \cap \mathbb{T} \neq \emptyset$.

Proof. It follows from theorem 52, proposition 6.1 (19) and the fact that every $\mathcal{E}$-recurrent operator is evidently recurrent.

\subsection{Weighted shifts satisfying property $\mathcal{P}_{\overline{\mathcal{B D}}}$}

In this section we will see some consequences of Theorem 52 in the frame of weighted backward shifts on $X=l_{p}\left(\mathbb{Z}_{+}\right),(1 \leq p<\infty)$ or $c_{0}\left(\mathbb{Z}_{+}\right)$. 


\subsubsection{A multiple recurrent operator which is not $\mathcal{E}$-recurrent}

Note that in particular, topologically $\mathcal{E}$-recurrence implies topologically multiply recurrence, however the converse is not true.

Proposition 54. Let $X=c_{0}\left(\mathbb{Z}_{+}\right)$or $X=l_{p}\left(\mathbb{Z}_{+}\right), 1 \leq p<\infty$, then there exists a weighted backward shift on $X$, which is topologically multiply recurrent but not topologically E-recurrent.

Then, we have the following diagram, where dashed arrow fails to hold.

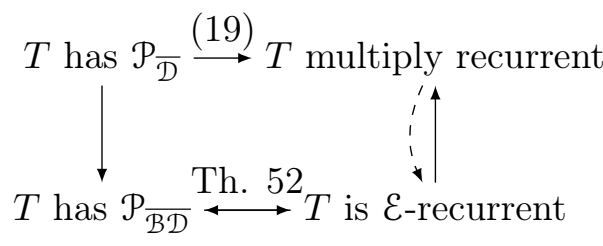

First, observe in the last picture that the converse of the main result of Costakis and Parissis (Theorem 50) cannot be obtained (i.e. there exists a multiple recurrent operator which does not have $\mathcal{P}_{\overline{\mathcal{D}}}$ ). On the other hand, Theorem 52 is in fact a generalization, as can be deduced from the diagram.

Now we proceed to prove Proposition 54 but first we need to point out the following observation. Obviously, condition

$$
\forall m \in \mathbb{N}, \sup _{n \in \mathbb{N}}\left\{\min _{1 \leq l \leq m}\left\{\left|w_{1} w_{2} \ldots w_{l n}\right|\right\}\right\}=\infty
$$

is equivalent to

$$
\forall M>0, \exists m_{0}: \forall m>m_{0}, \exists n: \min _{1 \leq l \leq m}\left\{\left|w_{1} w_{2} \ldots w_{l n}\right|\right\}>M .
$$

Then, by Proposition 5.3 (19) and Proposition 4.3 (16), we have the following: 


\section{RECURRENCE PROPERTIES DEFINED VIA ESSENTIAL IDEMPOTENTS OF $\beta \mathbb{N}$}

Proposition 55. Let $B_{w}$ a weighted backward shift on $X=c_{0}\left(\mathbb{Z}_{+}\right)$or $X=l_{p}\left(\mathbb{Z}_{+}\right), 1 \leq p<\infty$. The following are equivalent:

i) $B_{w}$ topologically multiply recurrent

ii) $\forall M>0, \exists m_{0}: \forall m>m_{0}, \exists n: \min _{1 \leq l \leq m}\left\{\left|w_{1} w_{2} \ldots w_{l n}\right|\right\}>M$.

Proof of Proposition 54

Proof. Note that for weighted backward shifts, being hypercyclic is equivalent to being recurrent (19). Then by Theorem 52, weighted backward shifts satisfying property $\mathcal{P}_{\overline{\mathcal{B D}}}$ are necessarily hypercyclic. Hence, thanks to Proposition 37 and Proposition 55 it suffices to find some $B_{w}$ that is non syndetic and satisfies 3.2.1.

Let us construct $\left(b_{n}\right)_{n \in \mathbb{N}}=B \subseteq \mathbb{N}$ with the property

$$
\forall m \in \mathbb{N}, \exists n \in \mathbb{N}: \ln \in B, \quad \forall 1 \leq l \leq m
$$

and define a weight $w=\left(w_{n}\right)_{n}$ in such a way that $A_{1}=\left\{n \in \mathbb{N}: \prod_{i=1}^{n}\left|w_{i}\right|>\right.$ 1 ) be non-syndetic and $w$ satisfies (3.2.1) on $B$. Denote $w^{n}=\left(w_{1}, \ldots, w_{n}\right)$. For better understanding we set $w_{n}^{*}$ for indicate that $n \in A_{2}$.

Let $m=1, n=1$ and define $b_{1}=1 \cdot 1=1$. Then, $w^{3}=\left(2^{*}, 1 / 2^{2}, 2\right)$. Let $m=2$, take $n=4$ and define $b_{2}=1 \cdot 4=4, b_{3}=2 \cdot 4=8$. Then, introducing an increasing gap on $A_{2}$ we set

$$
w^{b_{3}+3}=(\underbrace{2^{*}}_{w_{b_{1}}}, \frac{1}{2^{2}}, 2, \underbrace{2^{*}}_{w_{b_{2}}}, 2^{*}, 2^{*}, 2^{*}, \underbrace{2^{*}}_{w_{b_{3}}}, 1 / 2^{7}, 2,2) .
$$

Now, in order to satisfy (3.2.1) we must define $b_{4}$ at least equal to $b_{3}+$ $4+\left(b_{3}-b_{2}+1\right)=17$. Hence, for $m=3$, take $n=17$ and define $b_{4}=$ $1 \cdot 17=17, b_{5}=2 \cdot 17=34, b_{6}=3 \cdot 17=51$, introducing the corresponding increasing gap on $A_{2}$ we have

$$
w^{b_{6}+4}=(\underbrace{2^{*}}_{w_{b_{1}}}, \frac{1}{2^{2}}, 2, \underbrace{2^{*}}_{w_{b_{2}}}, \ldots, \underbrace{2^{*}}_{w_{b_{3}}}, 1 / 2^{7}, 2,2,2^{*}, \ldots, \underbrace{2^{*}}_{w_{b_{4}}}, \ldots, \underbrace{2^{*}}_{w_{b_{5}}}, \ldots, \underbrace{2^{*}}_{w_{b_{6}}},
$$




$$
\left.1 / 2^{43}, 2,2,2\right) \text {. }
$$

Again in order to satisfy (3.2.1) we must define $b_{7}$ at least equal to $b_{6}+$ $5+\left(b_{6}-\left(b_{3}+4\right)+1\right)=97$. Hence for $m=4$, take $n=97$ and define $b_{7}=1 \cdot 97=97, b_{8}=2 \cdot 97, b_{9}=3 \cdot 97, b_{10}=4 \cdot 97=388$, introducing the corresponding increasing gap on $A_{2}$ we have

$$
\begin{gathered}
w^{b_{10}+5}=(\underbrace{2^{*}}_{w_{b_{1}}}, \frac{1}{2^{2}}, 2, \underbrace{2^{*}}_{w_{b_{2}}}, \ldots, \underbrace{2^{*}}_{w_{b_{3}}}, 1 / 2^{7}, 2,2,2^{*}, \ldots, \underbrace{2^{*}}_{w_{b_{4}}}, \ldots, \underbrace{2^{*}}_{w_{b_{5}}}, \ldots, \underbrace{2^{*}}_{w_{b_{6}}}, 1 / 2^{43}, \\
2,2,2,2^{*}, \ldots, \underbrace{2^{*}}_{w_{b_{7}}}, \ldots, \underbrace{2^{*}}_{w_{b_{8}}}, \ldots \underbrace{2^{*}}_{w_{b_{9}}}, \underbrace{2^{*}}_{w_{b_{10}}}, 1 / 2^{337}, 2,2,2,2) .
\end{gathered}
$$

an so on. Clearly $B_{w}$ satisfies condition (3.2.1) and by Proposition 28 is not a syndetic operator.

\subsubsection{Further consequences}

In general, an operator satisfying property $\mathcal{P}_{\overline{\mathcal{B D}}}$ is not necessarily hypercyclic, consider for example the identity operator. But in the context of weighted backward shifts on $X=l_{p}$ or $c_{0}$, operators satisfying property $\mathcal{P}_{\overline{\mathcal{B D}}}$ are necessarily hypercyclic, even more, we show that they satisfy a stronger condition, i.e. $B_{w} \oplus B_{w}^{2} \oplus \ldots \oplus B_{w}^{r}$ is $\mathcal{E}^{*}$-operator on $X^{r}$, for any $r \in \mathbb{N}$.

Proposition 56. Let $w=\left(w_{k}\right)_{k \in \mathbb{Z}}$ be a bounded weight sequence and $B_{w} a$ bilateral weighted backward shift on $X=l_{p}(\mathbb{Z}), 1 \leq p \leq \infty$ or $c_{0}(\mathbb{Z})$. If $B_{w}$ satisfies property $\mathcal{P}_{\overline{\mathcal{B D}}}$ then $B_{w} \oplus B_{w}^{2} \oplus \ldots \oplus B_{w}^{r}$ is $\mathcal{E}^{*}$-operator on $X^{r}$, for any $r \in \mathbb{N}$.

Proof. Recall that

$$
A_{M ; j}=\left\{n \in \mathbb{N}: \prod_{i=j+1}^{j+n}\left|w_{i}\right|>M\right\}, \quad \bar{A}_{M ; j}=\left\{n \in \mathbb{N}: \frac{1}{\prod_{i=j-n+1}^{j}\left|w_{i}\right|}>M\right\} .
$$


Consider the filter

$$
\mathscr{G}=\left\{D \subseteq \mathbb{N}: \forall r \in \mathbb{N}, \exists W \in \mathcal{E}^{*}: l W \subseteq D, \forall l=1 \ldots r\right\} .
$$

Fact 1: If $B_{w}$ is topologically $\mathcal{E}$-recurrent then $A_{M ; j} \in \mathscr{G}$ and $\bar{A}_{M ; j} \in \mathscr{G}$, for any $j \in \mathbb{Z}$ and $M>0$.

Let $M>0, j \in \mathbb{Z}$. We must show

$$
\forall r \in \mathbb{N}, \quad \exists W \in \mathcal{E}^{*}: l k \in A_{M, j}, l k \in \bar{A}_{M, j} \quad \forall k \in W, \quad 1 \leq l \leq r .
$$

Let $r \in \mathbb{N}$. Pick $\delta>0$ such that $(1-\delta) / \delta>M$. Consider the open ball $B\left(e_{j}, \delta\right)=\left\{x \in X:\left\|x-e_{j}\right\|<\delta\right\} . B_{w}$ topologically $\mathcal{E}$-recurrent implies there exists $W \in \mathcal{E}^{*}$ such that for each $k \in W$ there exists

$$
y \in B\left(e_{j}, \delta\right)
$$

such that

$$
T^{l k} y \in B\left(e_{j}, \delta\right)
$$

for any $1 \leq l \leq r$. The existence of $W \in \mathcal{E}^{*}$ is due to the fact that we are considering $\left(\lambda_{n}\right)_{n}=1$ and hence in 3.0.4, $A$ can be taken as $\mathbb{N}$.

By 3.2 .2 ,

$$
\left|y_{j}-1\right|<\delta, \quad\left|y_{t}\right|<\delta \text { for } t \neq j
$$

By (3.2.3),

$$
\prod_{i=1}^{l k}\left|w_{i+j} y_{j+l k}-1\right|<\delta, \quad \prod_{i=1}^{l k}\left|w_{i+t} y_{t+l k}\right|<\delta \text { for } t \neq j .
$$

for any $1 \leq l \leq r$.

Now by (3.2.5), we have

$$
\left|\prod_{i=1}^{l k} w_{i+j} y_{j+l k}\right|>1-\delta .
$$


Thus by 3.2.4 and 3.2.6,

$$
\prod_{i=1}^{l k}\left|w_{i+j}\right|>\frac{1-\delta}{\delta}>M,
$$

and $l k \in A_{M, j}$, for any $1 \leq l \leq r$.

On the other hand, by 3.2.5,

$$
\prod_{i=j-l k+1}^{j}\left|w_{i} y_{j}\right|<\delta<\frac{1-\delta}{M} .
$$

Furthermore, by (3.2.4) we get

$$
\prod_{i=j-l k+1}^{j}\left|w_{i}\right|(1-\delta)<\prod_{i=j-l k+1}^{j}\left|w_{i} y_{j}\right|<\frac{1-\delta}{M} .
$$

Hence, $\prod_{i=j-l k+1}^{j}\left|w_{i}\right|<1 / M$ and $l k \in \bar{A}_{M, j}$, for any $1 \leq l \leq r$.

The following is a consequence of corollary 29:

Fact 2: If $B_{w}$ is such that $A_{M ; j} \in \mathscr{G}$ and $\bar{A}_{M ; j} \in \mathscr{G}$, for any $j \in \mathbb{Z}$ and $M>0$ then $B_{w} \oplus B_{w}^{2} \oplus \ldots \oplus B_{w}^{r}$ is $\mathcal{E}^{*}$-operator on $X^{r}$, for any $r \in \mathbb{N}$.

Now, we conclude our proof by theorem 52 , fact 1 and fact 2 .

With a similar proof we can obtain the following unilateral version of Proposition 56 .

Proposition 57. Let $w=\left(w_{n}\right)_{n \in \mathbb{Z}_{+}}$be a bounded weight sequence and $B_{w}$ an unilateral weighted backward shift on $X=l_{p}\left(\mathbb{Z}_{+}\right), 1 \leq p \leq \infty$ or $c_{0}\left(\mathbb{Z}_{+}\right)$. If $B_{w}$ satisfies property $\mathcal{P}_{\overline{\mathcal{B D}}}$ then $B_{w} \oplus B_{w}^{2} \oplus \ldots \oplus B_{w}^{r}$ is $\mathcal{E}^{*}$-operator on $X^{r}$, for any $r \in \mathbb{N}$.

Remark 58. The converse of Proposition 57 does not hold. Denote by $B(x ; r)$ the open ball centered at $x$ with radius $r$. The following was pointed out to me (personal communication) by Quentin Menet the following: 


\section{RECURRENCE PROPERTIES DEFINED VIA ESSENTIAL IDEMPOTENTS OF $\beta \mathbb{N}$}

Proposition 59. There exists a mixing weighted backward shift $B_{w}$ on $l_{p}\left(\mathbb{Z}_{+}\right)$such that $N\left(x, B\left(e_{0} ; 1 / 2\right)\right)$ has upper Banach density equals to zero for any $x \in l_{p}\left(\mathbb{Z}_{+}\right)$.

Now, let $B_{w}$ given by proposition 59 , then $B_{w}$ is mixing on $l_{p}\left(\mathbb{Z}_{+}\right)$and does not satisfy property $\mathcal{P}_{\overline{\mathcal{B D}}}$. Finally take into account the following fact proved in (14): $B_{w}$ is mixing if and only if $B_{w} \oplus B_{w}^{2} \oplus \ldots \oplus B_{w}^{r}$ is mixing, for any $r \in \mathbb{N}$, thus the converse of proposition 57 does not hold.

The proof of Proposition 59 is due to Quentin Menet and we include it here for the sake of completeness.

Proof. Let $B_{w}$ a weighted backward shift such that $\left|w_{n}\right| \geq 1$ for any $n \geq 1$ and suppose there exists $x \in l^{p}(\mathbb{N})$ and $m \geq 1$ such that

$$
\overline{B d}\left(N\left(x, B\left(e_{0} ; \frac{1}{2}\right)\right)\right)>\frac{1}{m} .
$$

We denote by $A$ the set $B\left(e_{0} ; 1 / 2\right)$. We have thus

$$
\lim _{s \rightarrow \infty} \limsup _{k \rightarrow \infty} \frac{|A \cap[k+1, k+s]|}{s}>\frac{1}{m} .
$$

In other words, there exists $s_{0} \geq 1$ such that for any $s \geq s_{0}$, any $k_{0} \geq 1$, there exists $k \geq k_{0}$ such that

$$
|A \cap[k+1, k+s]|>\frac{s}{m} .
$$

In particular, we obtain the existence of an integer $l_{0} \geq 1$ such that for any $l \geq l_{0}$, we can find an integer $k \geq 1$ satisfying

$$
|A \cap[k+1, k+l m]|>l .
$$

This means that for any $l \geq l_{0}$, there exist $n_{0}, \cdots, n_{l} \in[1, l m]$ such that for any $0 \leq j \leq l$,

$$
\left\|B_{w}^{k+n_{j}} x-e_{0}\right\|_{p}^{p}<\frac{1}{2^{p}} .
$$


We deduce that

$$
\sum_{j=1}^{l} \prod_{\nu=1}^{k+n_{0}}\left|w_{n_{j}-n_{0}+\nu} x_{k+n_{j}}\right|^{p}<\frac{1}{2^{p}}
$$

and that for any $0 \leq j \leq l$

$$
\prod_{\nu=1}^{k+n_{j}}\left|w_{\nu} x_{k+n_{j}}\right|>\frac{1}{2}
$$

We get by 3.2 .8

$\sum_{j=1}^{l} \prod_{\nu=1}^{k+n_{0}}\left|w_{n_{j}-n_{0}+\nu} x_{k+n_{j}}\right|^{p}=\sum_{j=1}^{l} \frac{\prod_{\nu=1}^{k+n_{j}}\left|w_{\nu}\right|^{p}}{\prod_{\nu=1}^{n_{j}-n_{0}}\left|w_{\nu}\right|^{p}}\left|x_{k+n_{j}}\right|^{p}>\sum_{j=1}^{l} \frac{1}{2^{p} \prod_{\nu=1}^{n_{j}-n_{0}}\left|w_{\nu}\right|^{p}}$

and thus by 3.2 .7

$$
\inf _{1 \leq j \leq l m} \frac{l}{\prod_{\nu=1}^{j}\left|w_{\nu}\right|^{p}} \leq \inf _{1 \leq j \leq l} \frac{l}{\prod_{\nu=1}^{n_{j}-n_{0}}\left|w_{\nu}\right|^{p}} \leq 2^{p} \sum_{j=1}^{l} \frac{1}{2^{p} \prod_{\nu=1}^{n_{j}-n_{0}}\left|w_{\nu}\right|^{p}}<1 .
$$

Hence, we conclude that there exists $m \geq 1$ such that

$$
\limsup _{l \rightarrow \infty} \frac{l}{\prod_{\nu=1}^{l m}\left|w_{\nu}\right|^{p}}<\infty
$$

because $\left|w_{n}\right| \geq 1$ for any $n \geq 1$.

Now, consider the weighted shift $B_{w}$ where $w_{\nu}=((\nu+1) / \nu)^{\frac{1}{2 p}}$. Since

$$
\prod_{\nu=1}^{n}\left|w_{\nu}\right|=(n+1)^{\frac{1}{2 p}} \rightarrow \infty
$$

the weighted shift $B_{w}$ is mixing on $l^{p}\left(\mathbb{Z}_{+}\right)$, see Chapter $4(24)$. On the other hand, $N\left(x, B\left(e_{0} ; 1 / 2\right)\right)$ has upper Banach density equals to zero for any $x \in l_{p}\left(\mathbb{Z}_{+}\right)$since for any $n \geq 1,\left|w_{n}\right| \geq 1$ and for any $m \geq 1$,

$$
\frac{l}{\prod_{\nu=1}^{l m}\left|w_{\nu}\right|^{p}}=\frac{l}{\sqrt{l m+1}} \rightarrow \infty \text {. }
$$




\section{RECURRENCE PROPERTIES DEFINED VIA ESSENTIAL IDEMPOTENTS OF $\beta \mathbb{N}$}

Proposition 41 shows that any reiteratively hypercyclic weighted backward shift on $c_{0}\left(\mathbb{Z}_{+}\right)$or $l_{p}\left(\mathbb{Z}_{+}\right)$is $\Delta^{*}$-operator. Now, with some additional hypothesis we will show that any weighted backward shift satisfying $\mathcal{P}_{\overline{\mathcal{B D}}}$ persists to be $\Delta^{*}$-operator.

Let $T$ topologically $\mathcal{E}$-recurrent, then by definition there exists some $p \in \mathcal{E}$ such that for any opene set $U$ in $X$, there exists $x \in X$ such that for any $r \in \mathbb{N}$,

$$
E(U)=\left\{k \in \mathbb{N}: \overline{B d}\left(M_{k, r}(U)\right)>0\right\} \in p
$$

where

$$
M_{k, r}(U)=\left\{a \in \mathbb{N}: T^{a} x \in \cap_{j=0}^{r} T^{-j k}(U)\right\} .
$$

Fix $U$ and $k$, then for any $n<r$ we have, $M_{k, r}(U) \subseteq M_{k, n}(U)$.

Proposition 60. Let $B_{w}$ satisfying that for any opene set $U$ in $X$, there exists $k \in E(U)$ for which $\bigcap_{r \in \mathbb{N}} M_{k, r}(U) \neq \emptyset$. If $B_{w}$ satisfies property $\mathcal{P}_{\overline{\mathcal{B D}}}$ then it is $\Delta^{*}$-operator.

Proof. Let $B_{w}$ satisfying property $\mathcal{P}_{\overline{\mathcal{B D}}}$ such that for each $U$ opene in $X$, there exists $k \in E(U)$ for which

$$
\bigcap_{r \in \mathbb{N}} M_{k, r}(U) \neq \emptyset \text {. }
$$

Observe that $B_{w}$ is an $\mathcal{E}$-recurrent weighted backward shift by theorem 52 . Fix $j \in \mathbb{N}$ and $M>0$, as in the proof of proposition 57, pick $\delta>0$ such that $(1-\delta) / \delta>M$ and consider the open ball $B\left(e_{j} ; \delta\right)$. Now by 3.2 .9 we can pick $\bar{a} \in \cap_{r \in \mathbb{N}} M_{k_{0}, r}\left(B\left(e_{j} ; \delta\right)\right)$ for some $k_{0} \in E\left(B\left(e_{j} ; \delta\right)\right)$. Then,

$$
y:=T^{\bar{a}} x \in \bigcap_{r \in \mathbb{N}} \bigcap_{l=0}^{r} T^{-l k_{0}}\left(B\left(e_{j}, \delta\right)\right) .
$$


On the other hand, 3.2.4 and 3.2.5 are valid for $k=k_{0}$, hence

$$
\left|\prod_{i=0}^{l k_{0}} w_{i+j}\right|>\frac{1-\delta}{\left|y_{j+l k_{0}}\right|}>\frac{1-\delta}{\delta}>M
$$

for any $l \in \mathbb{N}$. Then we can conclude that for any $M>0$ and any $j \in$ $\mathbb{N}$, there exists $k \in \mathbb{N}$ such that $k \mathbb{N}=\{k m: m \in \mathbb{N}\} \subseteq A_{M, j}$. Hence $A_{M, j} \in \Delta^{*}$, since every set of the form $k \mathbb{N}$ is a $\Delta^{*}$-set. In fact, fix $k \in \mathbb{N}$ and $A$ an infinite subset of $\mathbb{N}$. Pick any finite collection of $k+1$ elements in $A$ denoted by $a_{1}, a_{2}, \ldots, a_{k+1}$, hence necessarily there will be 2 elements $a_{i}, a_{j}$ with $1 \leq i, j \leq k+1$ such that $a_{i} \in k \mathbb{N}+t$ and $a_{j} \in k \mathbb{N}+t$ for some $0 \leq t \leq k-1$. Obviously, $a_{i}-a_{j} \in k \mathbb{N}$ and $k \mathbb{N} \cap(A-A) \neq \emptyset$, i.e. $k \mathbb{N} \in \Delta^{*}$.

\subsection{A characterization of reiteratively hypercyclic operators}

Using the same ideas of the proof of Theorem 52 we can obtain automatically more information about the return time set of a reiteratively hypercyclic operator respect to a reiteratively hypercyclic vector.

Definition 61. We will say that $T \in \mathcal{L}(X)$ is $\mathcal{E}$-reiteratively hypercyclic with respect to $\lambda=\left(\lambda_{n}\right)_{n}$ if there exists some $p \in \mathcal{E}$ and $x \in X$ such that for any opene set $U$ in $X$ and $r \in \mathbb{N}$, we have

$$
\left\{k \in \mathbb{N}: \overline{B d}\left(a \in \mathbb{N}: \lambda_{a} T^{a} x \in \cap_{j=0}^{r} T^{-j k}(U)\right)>0\right\} \in p .
$$

In the case $\left(\lambda_{n}\right)_{n}=1$, we simply say that $T$ is $\mathcal{E}$-reiteratively hypercyclic.

With the same sketch of the proof of Theorem 52 we can show the following: 


\section{RECURRENCE PROPERTIES DEFINED VIA ESSENTIAL IDEMPOTENTS OF $\beta \mathbb{N}$}

Theorem 62. Let $\left(\lambda_{n}\right)_{n}$ a sequence of non-zero complex numbers and some $p \in \mathcal{E}$ such that there exists $A \in p$ for which

$$
\overline{\mathcal{B D}}^{*}-\lim _{n}\left|\frac{\lambda_{n}}{\lambda_{n+k}}\right|=1, \forall k \in A
$$

then the family $\left(\lambda_{n} T^{n}\right)_{n}$ is reiteratively hypercyclic if and only if $T$ is $\mathcal{E}$ reiteratively hypercyclic with respect to $\lambda=\left(\lambda_{n}\right)_{n}$.

As a consequence we obtain a characterization of reiteratively hypercyclic operators.

Theorem 63. An operator $T \in \mathcal{L}(X)$ is reiteratively hypercyclic if and only if there exists some $x \in X$ such that for any opene set $U$ in $X$ and any $r \in \mathbb{N}$, we have

$$
\left\{k \in \mathbb{N}: \overline{B d}\left(a \in \mathbb{N}: T^{a} x \in T^{-k} U \cap \cdots \cap T^{-r k} U \cap U\right)>0\right\} \in \mathcal{E}^{*} .
$$

Proof. When considering $\left(\lambda_{n}\right)_{n}=1$, condition 3.3.1 holds with $A=\mathbb{N}$. The proof of Theorem 52 shows that in fact $\left\{k \in \mathbb{N}: \overline{B d}\left(a \in \mathbb{N}: T^{a} x \in\right.\right.$ $\left.\left.\cap_{j=0}^{r} T^{-j k}(U)\right)>0\right\}$ is $\mathcal{E}^{*}$-set.

\subsection{Questions}

It is not hard to see that chaotic operators, reiteratively hypercyclic operators, $\mathfrak{U}$-frequently hypercyclic operators and frequently hypercyclic operators satisfy property $\mathcal{P}_{\overline{\mathcal{B D}}}$. Consider $\mathcal{M} \subset \mathcal{L}(X), \mathcal{M} \neq \emptyset$. Now inspired in $[(25)$, Theorem 9], we pose the following questions:

Question 64. Does there exist some $\left(\lambda_{n}\right)_{n}$ such that $T \in \mathcal{M}$ is hypercyclic and topologically $\mathcal{E}$-recurrent with respect to $\left(\lambda_{n}\right)_{n}$ if and only if $T$ is chaotic? 
Question 65. Does there exist some $\left(\lambda_{n}\right)_{n}$ such that $T \in \mathcal{M}$ is hypercyclic and topologically $\mathcal{E}$-recurrent with respect to $\left(\lambda_{n}\right)_{n}$ if and only if $T$ is reiteratively hypercyclic?

Question 66. Does there exist some $\left(\lambda_{n}\right)_{n}$ such that $T \in \mathcal{M}$ is hypercyclic and topologically $\mathcal{E}$-recurrent with respect to $\left(\lambda_{n}\right)_{n}$ if and only if $T$ is $\mathfrak{U}$ frequently hypercyclic?

Question 67. Does there exist some $\left(\lambda_{n}\right)_{n}$ such that $T \in \mathcal{M}$ is hypercyclic and topologically $\mathcal{E}$-recurrent with respect to $\left(\lambda_{n}\right)_{n}$ if and only if $T$ is frequently hypercyclic? 
3. RECURRENCE PROPERTIES DEFINED VIA ESSENTIAL IDEMPOTENTS OF $\beta \mathbb{N}$ 


\section{4 \\ Disjoint hypercyclicity along filters}

The aim of this chapter is to prove that $B_{w}$ is $\mathscr{F}$-backward weighted shift if and only if the tuple $\left(B_{w}, \ldots, B_{w}^{r}\right)$ is $d-\mathscr{F}$, for any $r \in \mathbb{N}$ whenever $\mathscr{F}$ runs over some filters strictly containing the family of cofinite sets, as well as to verify that this statement fails beyond the weighted shift frame, by showing a mixing linear operator $T$ on a Hilbert space such that the tuple $\left(T, T^{2}\right)$ is not $d$-syndetic. Furthermore, we look into the relationship between reiteratively hypercyclic operators and $d-\mathscr{F}$ tuples, for filters $\mathscr{F}$ contained in the family of syndetic sets. Finally, recall that reiteratively hypercyclic operators are syndetic (Proposition 37) but the converse is not true (Proposition 42), hence we discuss under which conditions we can obtain reiterative hypercyclicity from syndeticity, for weighted backward shifts on $X=c_{0}\left(\mathbb{Z}_{+}\right)$or $l_{p}\left(\mathbb{Z}_{+}\right),(1 \leq p<\infty)$.

In a natural way we can introduce the notion of $\mathscr{F}$-disjoint transitivity 


\section{DISJOINT HYPERCYCLICITY ALONG FILTERS}

(or $d-\mathscr{F}$ for short).

Definition 68. The tuple of sequence of operators $\left(T_{1, n_{k}}, \ldots, T_{N, n_{k}}\right)_{k}$ is said to be $d$ - $\mathscr{F}$ if for any $N+1$-tuple $\left(U_{i}\right)_{i=0}^{N}$ of opene sets we have

$$
\left\{k \in \mathbb{N}: T_{1, n_{k}}^{-1}\left(U_{1}\right) \cap \cdots \cap T_{N, n_{k}}^{-1}\left(U_{N}\right) \cap U_{0} \neq \emptyset\right\} \in \mathscr{F} .
$$

In particular, when $T_{i, n_{k}}=T_{i}^{k}$, for any $k \in \mathbb{N}, 1 \leq i \leq N$ in the above definition, then the $N+1$-tuple of operators $\left(T_{1}, \ldots, T_{N}\right)$ is said to be $d$ - $\mathscr{F}$.

The case when $\mathscr{F}$ is the family of cofinite sets, i.e., $d$-mixing tuples of operators, has been studied in (14) . Let $X=c_{0}$ or $l_{p}, 1 \leq p<\infty$ and let $B_{w}$ be a weighted backward shift on $X$. Among other results, the authors of (14) prove that $B_{w}$ is mixing if and only if the tuple $\left(B_{w}, \ldots, B_{w}^{r}\right)$ is $d$-mixing, for any $r \in \mathbb{N}$.

\subsection{Tuples of powers of weighted shifts}

In this section we begin by studying conditions to impose on the weights in the search of a characterization of any tuple of powers of weighted shifts to be $d-\mathscr{F}$, with $\mathscr{F}$ a filter. This, together with a result of Ramsey theory about the preservation of certain notions of largeness in products, are the main ingredients of our main result: $B_{w}$ is $\mathscr{F}$-weighted backward shift if and only if $\left(B_{w}, \ldots, B_{w}^{r}\right)$ is $d$ - $\mathscr{F}$, for any $r \in \mathbb{N}$, where $\mathscr{F}$ runs along some filters containing strictly the family of cofinite sets.

Analogous to the $d$-Hypercyclicity Criterion for tuples of linear operators introduced in (16), we define the $d-\mathscr{F}$ Hypercyclicity Criterion, for $\mathscr{F}$ a family on $\mathbb{N}$. Let $X$ be a separable infinite dimensional Fréchet space. 


\subsection{Tuples of powers of weighted shifts}

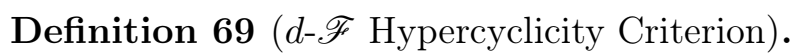

Let $\left(n_{k}\right)_{k}$ be a strictly increasing sequence of positive integers and $\mathscr{F}$ a family on $\mathbb{N}$. We say that a tuple of operators $\left(T_{1}, \ldots, T_{N}\right)$ in $\mathcal{L}(X)$ satisfies the $d$ - $\mathscr{F}$ Hypercyclicity Criterion with respect to $\left(n_{k}\right)_{k}$ provided there exist dense subsets $X_{0}, \ldots, X_{N}$ of $X$ and mappings $S_{l, k}: X_{l} \longrightarrow X,(1 \leq l \leq$ $N, k \in \mathbb{N}$ ) satisfying

(i) $\mathscr{F}-\lim _{k \rightarrow \infty} T_{l}^{n_{k}}(x)=0, \quad$ for any $x \in X_{0}$

(ii) $\quad \mathscr{F}-\lim _{k \rightarrow \infty} S_{l, k}(x)=0, \quad$ for any $x \in X_{l}(1 \leq l \leq N)$

(iii) $\mathscr{F}-\lim _{k \rightarrow \infty}\left(T_{l}^{n_{k}} S_{i, k}-\delta_{i, l} I d_{X_{l}}\right) x=0, \quad$ for any $x \in X_{l}(1 \leq i, l \leq N)$.

We say that $\left(T_{1}, \ldots, T_{N}\right)$ satisfies the $d$ - $\mathscr{F}$ Hypercyclicity Criterion if there exists some sequence $\left(n_{k}\right)_{k}$ for which $(i)-(i i i)$ is satisfied.

When $X_{s}=X_{l}$, for $0 \leq s, l \leq N$ and $\left(n_{k}\right)=(k)$ then we say that $\left(T_{1}, \ldots, T_{N}\right)$ satisfies the Original Kitai $d$ - $\mathscr{F}$ Criterion.

Connection between $d$ - $\mathscr{F}$ Hypercyclicity Criterion and $d$ - $\mathscr{F}$ tuples is the content of the following proposition that follows exactly the same sketch of proof of $[(16)$, Proposition 2.6].

Proposition 70. Let $\mathscr{F}$ be a filter on $\mathbb{N}$. If $\left(T_{1}, \ldots, T_{N}\right)$ satisfies the $d$ - $\mathscr{F}$ Hypercyclicity Criterion with respect to $\left(n_{k}\right)_{k}$, then the sequence $\left(T_{1}^{n_{k}}, \ldots, T_{N}^{n_{k}}\right)_{k}$ is $d-\mathscr{F}$.

Proof. Let $\left(V_{i}\right)_{i=0}^{N}$ be a $N$-tuple of opene subsets of $X$. Pick $y_{l} \in V_{l} \cap X_{l},(0 \leq$ $l \leq N)$ and $\epsilon>0$ such that $B\left(y_{l} ;(N+1) \epsilon\right) \subset V_{l}$, where $B(x ; r)$ denote the ball centered at $x$ with radius $r$. Set

$$
\begin{aligned}
& A=\left\{k \in \mathbb{N}: T_{l}^{n_{k}} y_{0} \in B(0 ; \epsilon)\right\} \in \mathscr{F} \\
& B_{l}=\left\{k \in \mathbb{N}: S_{l, k} y_{l} \in B(0 ; \epsilon)\right\} \in \mathscr{F}
\end{aligned}
$$


$C_{i, l}=\left\{k \in \mathbb{N}: T_{l}^{n_{k}} S_{i, k} y_{i}-\delta_{i, l} y_{i} \in B(0 ; \epsilon)\right\} \in \mathscr{F}, \quad(1 \leq i, l, \leq N)$.

But $\mathscr{F}$ is a filter, hence

$$
D:=A \cap \bigcap_{l=1}^{N}\left(B_{l} \cap \bigcap_{i=1}^{N} C_{i, l}\right) \in \mathscr{F} .
$$

Then, for each $k \in D$ we have $z_{k}:=y_{0}+\sum_{i=1}^{N} S_{i, k} y_{i} \in V_{0}$ and

$$
T_{l}^{n_{k}} z_{k}=T_{l}^{n_{k}} y_{0}+T_{l}^{n_{k}}\left(\sum_{i=1}^{N} S_{i, k} y_{i}\right)=T_{l}^{n_{k}} y_{0}+\sum_{i=1}^{N} T_{l}^{n_{k}} S_{i, k} y_{i} .
$$

On the other hand,

$$
\sum_{i=1}^{N} T_{l}^{n_{k}} S_{i, k} y_{i}=\left(T_{l}^{n_{k}} S_{l, k} y_{l}-y_{l}\right)+y_{l}+\sum_{\substack{i=l \\ i \neq l}}^{N} T_{l}^{n_{k}} S_{i, k} y_{i} \in B\left(y_{l} ; N \cdot \epsilon\right) .
$$

Hence, $T_{l}^{n_{k}} z_{k} \in B\left(y_{l} ;(N+1) \epsilon\right) \subset V_{l} \quad(1 \leq l \leq N)$. That is,

$$
D \subset\left\{k \in \mathbb{N}: V_{0} \cap T_{1}^{-n_{k}}\left(V_{1}\right) \cap \cdots \cap T_{N}^{-n_{k}}\left(V_{N}\right) \neq \emptyset\right\} .
$$

Hence, $\left(T_{1}^{n_{k}}, \ldots, T_{N}^{n_{k}}\right)$ is $d-\mathscr{F}$.

\subsubsection{Bilateral weighted shifts}

Let $X=c_{0}(\mathbb{Z})$ or $l_{p}(\mathbb{Z})(1 \leq p<\infty)$. For $l=1, \ldots, N$ consider $w_{l}=\left(w_{l, j}\right)_{j \in \mathbb{Z}}$ a bounded bilateral sequence of non-zero scalars and $B_{w_{l}}$ be the associated bilateral weighted backward shift on $X$ given by $B_{w_{l}} e_{k}=$ $w_{l, k} e_{k-1}(k \in \mathbb{Z})$, where $\left(e_{k}\right)_{k \in \mathbb{Z}}$ denotes the canonical basis of $X$ and $\left(e_{k}^{*}\right)_{k \in \mathbb{Z}}$ the associated sequence of coordinate functionals.

If $\left(B_{w_{1}}^{r_{1}}, \ldots, B_{w_{N}}^{r_{N}}\right)$ satisfies the d-Hypercyclicity Criterion then the integers $r_{1}, \ldots, r_{N}$ are necessarily distinct, see Corollary 3.4 (15). Then 


\subsection{Tuples of powers of weighted shifts}

since we are interested in tuples of weighted shifts satisfying a kind of $d$ Hypercyclicity Criterion along filters, we must consider distinct powers.

Let $1 \leq r_{1}<r_{2}<\cdots<r_{N}, M>0, j \in \mathbb{Z}$ and $1 \leq s<l \leq N$. Let $\left(n_{k}\right)_{k}$ be a strictly increasing sequence of positive integers. Set

$$
\begin{gathered}
A_{M, j, l,\left(n_{k}\right)}=\left\{k \in \mathbb{N}: \prod_{i=j+1}^{j+r_{l} n_{k}}\left|w_{l, i}\right|>M\right\} \\
\bar{A}_{M, j, l,\left(n_{k}\right)}=\left\{k \in \mathbb{N}: \frac{1}{\prod_{i=j-r_{l} n_{k}+1}^{j}\left|w_{l, i}\right|}>M\right\} \\
A_{M, j ;(s, l),\left(n_{k}\right)}=\left\{k \in \mathbb{N}: \frac{\prod_{i=j+1}^{j+r_{l} n_{k}}\left|w_{l, i}\right|}{\left.\prod_{i=j+\left(r_{l}-r_{s}\right) n_{k}+1}^{j+r_{l} n_{k}\left|w_{s, i}\right|}>M\right\}}\right. \\
\bar{A}_{M, j ;(s, l),\left(n_{k}\right)}=\left\{k \in \mathbb{N}: \frac{\prod_{i=j+1}^{j+r_{s} n_{k}}\left|w_{s, i}\right|}{\prod_{i=j-\left(r_{l}-r_{s}\right) n_{k}+1}^{j+r_{s} n_{k}}\left|w_{l, i}\right|}>M\right\} .
\end{gathered}
$$

If $\left(n_{k}\right)=(k)$, set $A_{M, j, l}, \bar{A}_{M, j, l}, A_{M, j ;(s, l)}, \bar{A}_{M, j ;(s, l)}$ respectively. We have the following:

Proposition 71. Let $\mathscr{F}$ be a filter on $\mathbb{N}$, then the following are equivalent:

(i) $\left(B_{w_{1}}^{r_{1} n_{k}}, \ldots, B_{w_{N}}^{r_{N} n_{k}}\right)_{k \in \mathbb{N}}$ is $d-\mathscr{F}$

(ii) for each $M>0, j \in \mathbb{Z}$ and $1 \leq s<l \leq N$, we have

$$
\begin{gathered}
A_{M, j, l,\left(n_{k}\right)} \in \mathscr{F}, \quad \bar{A}_{M, j, l,\left(n_{k}\right)} \in \mathscr{F} \\
A_{M, j ;(s, l),\left(n_{k}\right)} \in \mathscr{F}, \quad \bar{A}_{M, j ;(s, l),\left(n_{k}\right)} \in \mathscr{F}
\end{gathered}
$$

(iii) $\left(B_{w_{1}}^{r_{1}}, \ldots, B_{w_{N}}^{r_{N}}\right)$ satisfies the d-Fु Hypercyclicity Criterion with respect to $\left(n_{k}\right)_{k}$. 


\section{DISJOINT HYPERCYCLICITY ALONG FILTERS}

Proof. It is enough to consider $\left(n_{k}\right)_{k}=(k)$. The sketch of the proof is the same for any $\left(n_{k}\right)_{k}$. Denote

$$
N\left(V_{1}, \ldots, V_{N} ; V_{0}\right)=\left\{n \in \mathbb{N}: T_{1}^{-n} V_{1} \cap \cdots \cap T_{N}^{-n} V_{N} \cap V_{0} \neq \emptyset\right\}
$$

for any $N+1$-tuple $\left(V_{0}, \ldots, V_{N}\right)$ of opene sets of $X$.

(iii) implies (i) Follows by Proposition 70.

(i) implies (ii) Let $M>0, j \in \mathbb{Z}$ and $R>1$ such that $M R>1$.

Consider the opene set

$$
A_{R}=\left\{x \in X:\left|e_{j}^{*}(x)\right|>1 / R\right\} \cap\{x \in X:\|x\|<1\} .
$$

Let $V=\left\{x \in X:\left\|x-(M+1) e_{j}\right\|<\frac{1}{M R}\right\}$ and $m \in N(\underbrace{V, \ldots, V}_{N} ; A_{R})$. Pick $x \in A_{R}$, such that $B_{w_{l}}^{r_{l} m} x \in V$, where $1 \leq l \leq N$. Then

$$
\begin{aligned}
& \left|\prod_{i=j+1}^{j+r_{l} m} w_{l, i} x_{r_{l} m+j}-(M+1)\right|<\frac{1}{M R}<1, \quad 1 \leq l \leq N \\
& \prod_{i=t+1}^{t+r_{l} m}\left|w_{l, i} x_{r_{l} m+t}\right|<\frac{1}{M R}, \quad t \neq j, \quad 1 \leq l \leq N .
\end{aligned}
$$

We get by 4.1.1

$$
\prod_{i=j+1}^{j+r_{l} m}\left|w_{l, i}\right|>\prod_{i=j+1}^{j+r_{l} m}\left|w_{l, i} x_{r_{l} m+j}\right|>M, \quad 1 \leq l \leq N .
$$

Now, by 4.1.2 we obtain $\prod_{i=j-r_{l} m+1}^{j}\left|w_{l, i} x_{j}\right|<\frac{1}{M R}$, hence

$$
\prod_{i=j-r_{l} m+1}^{j}\left|w_{l, i}\right| \cdot \frac{1}{R}<\prod_{i=j-r_{l} m+1}^{j}\left|w_{l, i} x_{j}\right|<\frac{1}{M R} .
$$


Thus

$$
\prod_{i=j-r_{l} m+1}^{j}\left|w_{l, i}\right|<\frac{1}{M}
$$

Again by 4.1.1 and 4.1.2 we get

$$
\frac{\prod_{i=j+1}^{j+r_{l} m}\left|w_{l, i}\right|}{\prod_{i=j+\left(r_{l}-r_{s}\right) m+1}^{j+r_{l} m}\left|w_{s, i}\right|}>\prod_{i=j+1}^{j+r_{l} m}\left|w_{l, i} x_{j+r_{l} m}\right|>M, \quad 1 \leq s<l \leq N .
$$

Finally, for $\quad 1 \leq s<l \leq N$

$$
\frac{\prod_{i=j+1}^{j+r_{s} m}\left|w_{s, i}\right|}{\prod_{i=j-\left(r_{l}-r_{s}\right) m+1}^{j+r_{s} m}\left|w_{l, i}\right|}=\frac{\prod_{i=j+1}^{j+r_{s} m}\left|w_{s, i} \cdot x_{j+r_{s} m}\right|}{\prod_{i=j-\left(r_{l}-r_{s}\right) m+1}^{j+r_{s} m}\left|w_{l, i} \cdot x_{j+r_{s} m}\right|}>\frac{M}{1 / M R}>M .
$$

(ii) implies (iii) Let $X_{0}=\cdots=X_{N}$, the set of all finitely supported vectors in $X$ and $S_{l, k}: X_{0} \longrightarrow X,(1 \leq l \leq N)$ be defined as

$$
S_{l, k} e_{t}=\frac{1}{\prod_{i=t+1}^{t+r_{l} k} w_{l, i}} e_{t+r_{l} k}, \quad k \in \mathbb{Z} .
$$

a) Let us verify that $\mathscr{F}-\lim _{k \rightarrow \infty} B_{w_{l}}^{r_{l} k}(x)=0$, for every $x \in X_{0}$.

Let $x \in X_{0}$, denote $F=\left\{j \in \mathbb{Z}: x_{j} \neq 0\right\}$. Recall that the $\left(j-r_{l} k\right)-$ th coefficient of $B_{w_{l}}^{r_{l} k} x$ is equal to $\prod_{i=j-r_{l} k+1}^{j} w_{l, i} x_{j}$. Let $M>0$, then

$$
\bigcap_{j \in F}\left\{k \in \mathbb{N}: \prod_{i=j-r_{l} k+1}^{j}\left|w_{l, i}\right|<\frac{1}{M\|x\|}\right\} \subset\left\{k \in \mathbb{N}:\left\|B_{w_{l}}^{r_{l} k} x\right\|<\frac{1}{M}\right\},
$$

hence

$$
\bigcap_{j \in F} \bar{A}_{M\|x\|, j ; l} \subseteq\left\{k \in \mathbb{N}:\left\|B_{w_{l}}^{r_{l} k} x\right\|<\frac{1}{M}\right\}
$$

and by hypothesis $\bigcap_{j \in F} \bar{A}_{M\|x\|, j ; l} \in \mathscr{F}$ for any $M>0$. Obviously $B_{w_{l}}^{r_{l} k} S_{l, k}=$ $I d_{X_{0}}$, for any $k$.

b) Let us verify that $\mathscr{F}-\lim _{k \rightarrow \infty} S_{l, k}(x)=0$ for any $x \in X_{0}$. 


\section{DISJOINT HYPERCYCLICITY ALONG FILTERS}

Let $x \in X_{0}, F=\left\{j \in \mathbb{Z}: x_{j} \neq 0\right\}, \epsilon>0$. Set $M_{j}=\frac{1}{\epsilon} \cdot|F|^{1 / p}\left|x_{j}\right|$ whenever $X=l_{p}(\mathbb{Z})$ or $M_{j}=\left|x_{j}\right| / \epsilon$ whenever $X=c_{0}(\mathbb{Z})$. Set $M=$ $\max _{j \in F}\left|M_{j}\right|$, then for each $k \in \cap_{j \in F} A_{M, j, l}$ it results $\left\|S_{l, k}(x)\right\|<\epsilon$, i.e.

$$
\left\{k \in \mathbb{N}:\left\|S_{l, k}(x)\right\|<\epsilon\right\} \in \mathscr{F} .
$$

c) Let $1 \leq s<l \leq N$, then

$$
B_{w_{l}}^{r_{l} k} S_{s, k} e_{t}=\frac{1}{\prod_{i=t+1}^{t+r_{s} k} w_{s, i}} B_{w_{l}}^{r_{l} k} e_{t+r_{s} k}=\frac{\prod_{i=t+r_{s} k}^{t+\left(r_{s}-r_{l}\right) k+1} w_{l, i}}{\prod_{i=t+1}^{t+r_{s} k} w_{s, i}} e_{t+\left(r_{s}-r_{l}\right) k} .
$$

Now, arguing as in $b$ ), the last equation implies that $\mathscr{F}-\lim _{k \rightarrow \infty} B_{w_{l}}^{r_{l} k} S_{s, k} x=$ 0 , for every $x \in X_{0}$, since $\mathscr{F}$ is a filter and $\bar{A}_{M, t ;(s, l)} \in \mathscr{F}$ for any $M>$ $0, t \in \mathbb{Z}$.

d) Let $1 \leq s<l \leq N$, then

$$
B_{w_{s}}^{r_{s} k} S_{l, k} e_{t}=\frac{1}{\prod_{i=t+1}^{t+r_{l} k} w_{l, i}} B_{w_{s}}^{r_{s} k} e_{t+r_{l} k}=\frac{\prod_{i=t+r_{l} k}^{t+\left(r_{l}-r_{s}\right) k+1} w_{s, i}}{\prod_{i=t+1}^{t+r_{l} k} w_{l, i}} e_{t+\left(r_{l}-r_{s}\right) k} .
$$

Again, arguing as in $b$ ), the last equation implies that $\mathscr{F}-\lim _{k \rightarrow \infty} B_{w_{s}}^{r_{s} k} S_{l, k} x=$ 0 , for every $x \in X_{0}$, since $\mathscr{F}$ is a filter and $A_{M, t ;(s, l)} \in \mathscr{F}$ for any $M>$ $0, t \in \mathbb{Z}$. We can conclude that $\left(B_{w_{1}}^{r_{1}}, \ldots, B_{w_{N}}^{r_{N}}\right)$ satisfy the $d-\mathscr{F}$ Hypercyclicity Criterion with respect to $(k)$.

Now, using Proposition 71 and adapting Theorem 2.5 (35) to bilateral weighted backward shifts, we obtain the following:

Corollary 72. Let $X=c_{0}(\mathbb{Z})$ or $l_{p}(\mathbb{Z})(1 \leq p<\infty), w=\left(w_{j}\right)_{j \in \mathbb{Z}}$ a bounded bilateral weight sequence, $\mathscr{F}$ a filter on $\mathbb{N}$ and $r_{0}=0<1 \leq r_{1}<\cdots<r_{N}$, then the following are equivalent:

(i) $\left(B_{w}^{r_{1}}, \ldots, B_{w}^{r_{N}}\right)$ is $d-\mathscr{F}$ 


\subsection{Tuples of powers of weighted shifts}

(ii) $\left(B_{w}^{r_{1}}, \ldots, B_{w}^{r_{N}}\right)$ satisfies the $d-\mathscr{F}$ Hypercyclicity Criterion with respect to $(k)$

(iii) for any $M>0, j \in \mathbb{Z}$ and $0 \leq s<l \leq N$ we have

$$
\left\{m \in \mathbb{N}: \prod_{i=j+1}^{j+\left(r_{l}-r_{s}\right) m}\left|w_{i}\right|>M\right\} \in \mathscr{F}
$$

and

$$
\left\{m \in \mathbb{N}: \frac{1}{\prod_{i=j-\left(r_{l}-r_{s}\right) m+1}^{j}\left|w_{i}\right|}>M\right\} \in \mathscr{F}
$$

(iv) $\oplus_{0 \leq s<l \leq N} B_{w}^{\left(r_{l}-r_{s}\right)}$ is an $\mathscr{F}$-operator on $X^{\frac{N(N+1)}{2}}$.

Remark 73. Observe that the conclusion of Corollary 72 does not hold in general, for bounded linear operators. In fact, for $r_{i}=i, 1 \leq i \leq N$. Bès, Martin, Peris and Shkarin (14), have proved that $\left(I+B_{w}, \ldots,\left(I+B_{w}\right)^{r}\right)$ is $d$-J* (i.e. $d$-mixing). On the other hand, by a result of Grivaux (23), $I+B_{w}$ does not satisfy the $\mathcal{J}^{*}$-Hypercyclicity Criterion with respect to $(k)$, for $B_{w}$ weighted backward shift on $l_{p}$ and $\left(w_{n}\right)$ a decreasing sequence of positive weights such that $\lim _{n \rightarrow \infty} n\left(\prod_{i=1}^{n} w_{i}\right)^{1 / n}=0$. Hence, $(i)$ and (ii) in Corollary 72 are not equivalent beyond the weighted shift frame. Moreover, the same situation take place between $(i)$ and $(i v)$, since Shkarin has exhibited a bounded linear operator $T$ such that $T \oplus T^{2}$ is mixing but $\left(T, T^{2}\right)$ is not $d$-mixing.

We state the main result of this section:

Theorem 74. Let $\mathscr{F}$ be any of $\Delta^{*}, \mathrm{JP}^{*}$ or $\mathcal{P S} \mathcal{S}^{*}$. Let $X=c_{0}(\mathbb{Z})$ or $l_{p}(\mathbb{Z}),(1 \leq$ $p<\infty)$ and let $\left(w_{k}\right)_{k \in \mathbb{Z}}$ be a bounded bilateral weight sequence. Then for any $r \in \mathbb{N}$, the following are equivalent:

(i) $B_{w}$ is an $\mathscr{F}$-operator 


\section{DISJOINT HYPERCYCLICITY ALONG FILTERS}

(ii) for any $M>0, j \in \mathbb{Z}$ and $t=1, \ldots, r$

$\left\{m \in \mathbb{N}: \prod_{i=j+1}^{j+t m}\left|w_{i}\right|>M\right\} \in \mathscr{F}, \quad\left\{m \in \mathbb{N}: \frac{1}{\prod_{i=j-t m+1}^{j}\left|w_{i}\right|}>M\right\} \in \mathscr{F}$

(iii) $\left(B_{w}, \ldots, B_{w}^{r}\right)$ is $d-\mathscr{F}$

(iv) $B_{w} \oplus \cdots \oplus B_{w}^{r}$ is an $\mathscr{F}$-operator on $X^{r}$.

Recall that $\mathcal{J}^{*}$-operators are commonly known as mixing operators. Obviously, mixing operators are $\Delta^{*}$-operators, but the converse is not true as exhibited in Proposition 33 and the example is a weighted shift. Therefore, the conclusion of Theorem 74 does not necessarily follows from the statement: $B_{w}$ is mixing if and only if $\left(B_{w}, \ldots, B_{w}^{r}\right)$ is $d$-mixing, for any $r \in \mathbb{N}$, shown in (14).

Proof of Theorem 74

Proof. (i) implies (ii) Denote $A_{M ; j}=\left\{m \in \mathbb{N}:\left|\prod_{i=j+1}^{j+m} w_{i}\right|>M\right\}$ and $\bar{A}_{M ; j}=\left\{m \in \mathbb{N}: \frac{1}{\prod_{i=j-m+1}^{j}\left|w_{i}\right|}>M\right\}$. If $B_{w}$ is $\mathscr{F}$-operator then $A_{M ; j} \in \mathscr{F}$ and $\bar{A}_{M ; j} \in \mathscr{F}$ for any $M>0, j \in \mathbb{Z}$ by Corollary 72 with $N=1$ and $r_{1}=1$. Let $r \in \mathbb{N}$, then by Corollary 2.3 (9) and corollary $2.7(9)$ we have

$$
\begin{array}{r}
A=\{m, 2 m, \ldots, r m: m \in \mathbb{N}\} \cap(\underbrace{A_{M ; j} \times \cdots \times A_{M ; j}}_{r-\text { times }}) \in \mathscr{F} \\
\bar{A}=\{m, 2 m, \ldots, r m: m \in \mathbb{N}\} \cap(\underbrace{\bar{A}_{M ; j} \times \cdots \times \bar{A}_{M ; j}}_{r \text { times }}) \in \mathscr{F}
\end{array}
$$

in $\{m, 2 m, \ldots, r m: m \in \mathbb{N}\}$.

Denote $\prod_{i}$ the projection onto the $i$-th coordinate. It is not difficult to see that $\prod_{1}(A) \in \mathscr{F}$ and $\prod_{1}(\bar{A}) \in \mathscr{F}$ in $\mathbb{N}$. Then, 4.1 .3 is equivalent to say

$$
\left\{m \in \mathbb{N}: t m \in A_{M ; j}\right\} \in \mathscr{F}
$$




$$
\left\{m \in \mathbb{N}: t m \in \bar{A}_{M ; j}\right\} \in \mathscr{F}
$$

for any $M>0, j \in \mathbb{Z}$ and $t=1, \ldots, r$.

(ii), (iii) and (iv) are equivalent by Corollary 72

(iii) implies (i) Obvious.

Notice that the family of syndetic sets is not a filter, since $2 \mathbb{N}$ and $2 \mathbb{N}+1$ are syndetic sets but disjoint. Nevertheless, at the level of operators, we know that syndetic operators behave as filter-operators in virtue of their equivalence with $\mathcal{P S}^{*}$-operators (Proposition 22). As expected, Theorem 74 is also valid for $\mathscr{F}=\mathcal{S}$.

Corollary 75. Let $X=c_{0}(\mathbb{Z})$ or $l_{p}(\mathbb{Z})(1 \leq p<\infty)$, a bilateral bounded

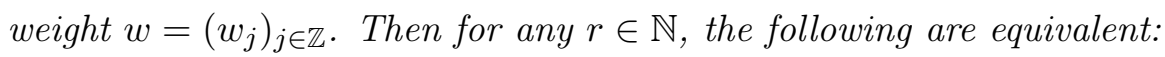

(i) $B_{w}$ is syndetic weighted backward shift

(ii) $\left(B_{w}, \ldots, B_{w}^{r}\right)$ is $d-\mathcal{S}$

(ii) $\left(B_{w}, \ldots, B_{w}^{r}\right)$ is d-PS*

(iv) $B_{w} \oplus \cdots \oplus B_{w}^{r}$ is a syndetic operator on $X^{r}$.

Corollary 75 follows from Theorem 74 and the equivalence between syndetic and $\mathcal{P S}^{*}$-operators (Proposition 22).

\subsubsection{Unilateral weighted shifts}

Let $X=c_{0}\left(\mathbb{Z}_{+}\right)$or $l_{p}\left(\mathbb{Z}_{+}\right)(1 \leq p<\infty)$. For $l=1, \ldots, N$ let $w_{l}=$ $\left(w_{l, n}\right)_{n \in \mathbb{N}}$ a bounded unilateral weight sequence of non-zero scalars and $B_{w_{l}}$ be the associated unilateral weighted backward shift on $X$ given by $B_{w_{l}} e_{n}:=$ $w_{l, n} e_{n-1}, n \geq 1$ with $B_{w_{l}} e_{0}:=0$, where $\left(e_{n}\right)_{n \in \mathbb{Z}_{+}}$denotes the canonical basis of $X$ and $\left(e_{n}^{*}\right)_{n \in \mathbb{Z}_{+}}$the associated sequence of coordinate functionals. 


\section{DISJOINT HYPERCYCLICITY ALONG FILTERS}

Consider $1 \leq r_{1}<r_{2}<\cdots<r_{N}$. The following is the unilateral version of Proposition 71

Proposition 76. Let $\mathscr{F}$ be a filter on $\mathbb{N}$, then the following are equivalent:

(i) $\left(B_{w_{1}}^{r_{1} n_{k}}, \ldots, B_{w_{N}}^{r_{N} n_{k}}\right)_{k \in \mathbb{N}}$ is $d-\mathscr{F}$

(ii) for each $M>0, j \in \mathbb{Z}_{+}, 1 \leq s<l \leq N$ we have

$$
A_{M, j, l,\left(n_{k}\right)} \in \mathscr{F}
$$

and

$$
A_{M, j ;(s, l),\left(n_{k}\right)} \in \mathscr{F}
$$

(iii) $\left(B_{w_{1}}^{r_{1}}, \ldots, B_{w_{N}}^{r_{N}}\right)$ satisfies the d-FुF Hypercyclicity Criterion with respect to $\left(n_{k}\right)$.

Proposition 76 follows in a similar way as Theorem 71 .

Recall that $P_{f}\left(\mathbb{Z}_{+}\right)=\left\{A \subset \mathbb{Z}_{+}:|A|<\infty\right\}$.

Corollary 77. Let $0=r_{0}<1 \leq r_{1}<\cdots<r_{n}$. Let $\mathscr{F}$ be a filter on $\mathbb{N}$ and let $w=\left(w_{n}\right)_{n \in \mathbb{Z}_{+}}$be a bounded unilateral weight sequence. Then the following are equivalent:

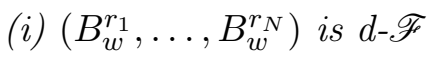

(ii) $\left(B_{w}^{r_{1}}, \ldots, B_{w}^{r_{N}}\right)$ satisfies the $d$-Fु Hypercyclicity Criterion with respect to $(k)$

(iii) $\oplus_{0 \leq s<l \leq N} B_{w}^{\left(r_{l}-r_{s}\right)}$ is an $\mathscr{F}$-operator on $X^{\frac{N(N+1)}{2}}$

(iv) for any $M>0, j \in \mathbb{Z}_{+}$and $0 \leq s<l \leq N$ it holds

$$
\left\{m \in \mathbb{N}: \prod_{i=j+1}^{j+m\left(r_{l}-r_{s}\right)}\left|w_{i}\right|>M\right\} \in \mathscr{F} .
$$

In addition, 
- If $\mathscr{F}$ is a shift invariant, then each condition $(i)-(i v)$ is equivalent to

$$
\left(i v^{\prime}\right) \quad\left\{m \in \mathbb{N}: \prod_{i=1}^{m\left(r_{l}-r_{s}\right)}\left|w_{i}\right|>M\right\} \in \mathscr{F}
$$

for any $M>0$ and $0 \leq s<l \leq N$.

Proof. (i) - (iv) are equivalent, follows the same steps as Corollary 72 adapted to the unilateral case. Moreover, suppose $\mathscr{F}$ is a shift invariant filter, let us see that $\left(i v^{\prime}\right)$ implies $(i v)$. Let $M>0, j \in \mathbb{Z}_{+}, 0 \leq s<l \leq N$ and $k=\max _{t \in \mathbb{N}}\left|w_{t}\right|$. It is enough to verify

$$
\left\{n \in \mathbb{N}: \prod_{i=1}^{\left(r_{l}-r_{s}\right) n}\left|w_{i}\right|>M k^{\left(r_{l}-r_{s}\right) j}\right\}-j \subseteq\left\{n \in \mathbb{N}: \prod_{i=j+1}^{j+\left(r_{l}-r_{s}\right) n}\left|w_{i}\right|>M\right\} .
$$

In fact, let $m \in \mathbb{N}$ such that $\prod_{i=1}^{\left(r_{l}-r_{s}\right) m}\left|w_{i}\right|>M k^{\left(r_{l}-r_{s}\right) j}$ then

$$
\prod_{i=j+1}^{j+\left(r_{l}-r_{s}\right)(m-j)}\left|w_{i}\right|=\frac{\prod_{i=1}^{\left(r_{l}-r_{s}\right) m}\left|w_{i}\right|}{\prod_{i=1}^{j}\left|w_{i}\right| \cdot \prod_{i=\left(r_{l}-r_{s}\right)(m-j)+j+1}^{\left(r_{l}-r_{s}\right) m}\left|w_{i}\right|}>\frac{M k^{\left(r_{l}-r_{s}\right) j}}{k^{\left(r_{l}-r_{s}\right) j}}=M .
$$

A similar result to Theorem 74 holds for unilateral weighted shifts.

Theorem 78. Let $\mathscr{F}=\Delta^{*}, \mathcal{J P}^{*}, \mathcal{P S} *$ Let $X=c_{0}\left(\mathbb{Z}_{+}\right)$or $l_{p}\left(\mathbb{Z}_{+}\right)(1 \leq p<$ $\infty)$ and $\left(w_{n}\right)_{n \in \mathbb{Z}_{+}}$a bounded unilateral weight. Then for any $r \in \mathbb{N}$, the following are equivalent:

(i) $B_{w}$ is $\mathscr{F}$-operator

(ii) for any $M>0, j \in \mathbb{Z}_{+}, t=1, \ldots, r$

$$
\left\{m \in \mathbb{N}: \prod_{i=j+1}^{j+t m}\left|w_{i}\right|>M\right\} \in \mathscr{F}
$$




\section{DISJOINT HYPERCYCLICITY ALONG FILTERS}

(iii) $\left(B_{w}, \ldots, B_{w}^{r}\right)$ is $d-\mathscr{F}$

(iv) $B_{w} \oplus \cdots \oplus B_{w}^{r}$ is $\mathscr{F}$-operator on $X^{r}$.

Proof. Analogous to the proof of Theorem 74 with the aid of Corollary 77

Corollary 79. Let $X=c_{0}\left(\mathbb{Z}_{+}\right)$or $l_{p}\left(\mathbb{Z}_{+}\right)(1 \leq p<\infty)$, an unilateral bounded weight $w=\left(w_{n}\right)_{n \in \mathbb{Z}_{+}}$. Then for any $r \in \mathbb{N}$, the following are equivalent:

(i) $B_{w}$ is syndetic weighted backward shift

(ii) $\left(B_{w}, \ldots, B_{w}^{r}\right)$ is $d-\mathcal{S}$

(iii) $\left(B_{w}, \ldots, B_{w}^{r}\right)$ is d-PS*

(iv) $B_{w} \oplus \cdots \oplus B_{w}^{r}$ is syndetic weighted backward shift on $X^{r}$.

Proof. Follows from Theorem 78 and the equivalence between syndetic and PS*-operators.

As a consequence we can say a little more about the examples given in propositions 31,32 and 33 concerning the existence of a $\mathcal{J P}^{*} \backslash \Delta^{*}$-operator, $\mathcal{S} \backslash \mathcal{J P}^{*}$-operator and $\Delta^{*}$ but not mixing operator.

Proposition 80. Let $X=c_{0}\left(\mathbb{Z}_{+}\right)$or $l_{p}\left(\mathbb{Z}_{+}\right)(1 \leq p<\infty)$. For any $r \in \mathbb{N}$ the following holds

(i) There exists a weighted backward shift $B_{w}$ on $X$ such that $\left(B_{w}, \ldots, B_{w}^{r}\right)$ is $d$-JP范 but $B_{w}$ is not $\Delta^{*}$-operator

(ii) There exists a weighted backward shift $B_{w}$ on $X$ such that $\left(B_{w}, \ldots, B_{w}^{r}\right)$ is d-PS* but $B_{w}$ is not $\mathcal{J P}^{*}$-operator

(iii) There exists a weighted backward shift $B_{w}$ on $X$ such that $\left(B_{w}, \ldots, B_{w}^{r}\right)$ is $d-\Delta^{*}$ but $B_{w}$ is not mixing operator. 


\subsection{Tuples of powers of weighted shifts}

Proof. The examples given in propositions 31, 32 and 33 are weighted backward shifts on $X$. The conclusions follow by Theorem 78 and Corollary 79

This is in sharp contrast to what happens with scalar multiples of powers of the unilateral unweighted backward shift $B$. In fact, $\left(\lambda_{1} B^{r_{1}}, \ldots, \lambda_{N} B^{r_{N}}\right)$ is $d$-hypercyclic on $l_{2}$ if and only if $\left(\lambda_{1} B^{r_{1}}, \ldots, \lambda_{N} B^{r_{N}}\right)$ is $d$-mixing on $l_{2}$ if and only if $1 \leq r_{1}<r_{2}<\cdots<r_{N}$ and $1<\left|\lambda_{1}\right|<\cdots<\left|\lambda_{N}\right|$ as shown in Corollary 4.7 (15).

Remark 81. Let $X=c_{0}(\mathbb{Z})$ or $l_{p}(\mathbb{Z})\left(c_{0}\left(\mathbb{Z}_{+}\right)\right.$or $\left.l_{p}\left(\mathbb{Z}_{+}\right)\right)$, Theorem 74 (respectively, Theorem 78) can be obtained in another way. In fact, in its proof we have used Proposition 71 (respectively, Proposition 76) which gives us conditions on the weight $w$ in such a way that the tuple $\left(B_{w}, \ldots, B_{w}^{r}\right)$ is $d$ $\mathscr{F}$, with $r \in \mathbb{N}$ and $\mathscr{F}$ a filter on $\mathbb{N}$. But we can exploit another information coming from the proof of Proposition 71 (respectively, Proposition 76) and is the fact that any $\mathscr{F}$-weighted backward shift on $X$ satisfies the Original Kitai $\mathscr{F}$-criterion. Now, an adaptation to filters of Theorem 3.4 (14) and a further application of Corollary 2.3 (9) and Corollary 2.7 (9) allows us to conclude as in Theorem 74 (respectively, Theorem 78), that is, $B_{w}$ is $\mathscr{F}$-weighted backward shift if and only if $\left(B_{w}, \ldots, B_{w}^{r}\right)$ is $d$ - $\mathscr{F}$.

Theorem 82. Let $T \in \mathcal{L}(X)$, where $X$ is a topological vector space, $\mathscr{F}=$ $\Delta^{*}, \mathcal{J P}^{*}, \mathcal{P S}^{*}$. If $T$ satisfies the Original Kitai $\mathscr{F}$-criterion then $\left(T, \ldots, T^{r}\right)$ is $d-\mathscr{F}$, for any $r \in \mathbb{N}$.

Proof. Make the adaptation to filters of [(14), Theorem 3.4] and apply [(9), Corollary 2.3] and [(9), Corollary 2.7]. Details are left to the reader. 


\section{DISJOINT HYPERCYCLICITY ALONG FILTERS}

\subsubsection{The non-filter case}

Everything changes when dealing with non-filter families, with the exception of the family of syndetic sets. A slight modification of Example 4.5 (16) give us an immediate answer.

Proposition 83. Let $X=c_{0}\left(\mathbb{Z}_{+}\right)$or $l_{p}\left(\mathbb{Z}_{+}\right)(1 \leq p<\infty)$, then there exists a thick backward weighted shift $B_{w}$ (i.e. a weakly mixing backward weighted shift) such that

- $\left(B_{w}, B_{w}^{2}\right)$ is not d-topologically transitive. In particular, $B_{w}$ is $\mathscr{F}$ operator but $\left(B_{w}, B_{w}^{2}\right)$ is not $d-\mathscr{F}$, for $\mathscr{F}=\Delta, \mathcal{J P}, \mathcal{P S}, \mathcal{T}$

- $\left(B_{w}, B_{w}^{2}\right)$ satisfies the d-thick Hypercyclicity Criterion with respect to $(k)$.

Recall that any tuple of powers of a fixed backward weighted shift on $c_{0}$ or $l_{p}$ is $d$-transitive if and only if it is $d$-hypercyclic if and only if it satisfies the $d$-J* Hypercyclicity Criterion with respect to some $\left(n_{k}\right)$. This follows by Proposition 2.3, Theorem 2.7 and Theorem 4.1, in (16). Hence, in this case, $\left(B_{w}, B_{w}^{2}\right)$ does not satisfy the $d$-J* Hypercyclicity Criterion with respect to any $\left(n_{k}\right)$. In other words, $\left(B_{w}, B_{w}^{2}\right)$ does not satisfy the $d$-Hypercyclicity Criterion, though it satisfies the $d$-thick Hypercyclicity Criterion with respect to $(k)$.

Proof. Let $w=\left(w_{k}\right)_{k \in \mathbb{Z}_{+}}$defined as

$$
w_{k}=\left\{\begin{array}{cl}
2 & \text { if } k \in \bigcup_{n \in \mathbb{N}}\left\{2^{2 n}+1, \ldots, 2^{2 n}+2 n\right\} \\
\frac{1}{2^{2 n}} & \text { if } k=2^{2 n}+2 n+1 \quad \text { for some } n \in \mathbb{N} \\
1 & \text { otherwise. }
\end{array}\right.
$$


Denote $A_{M}:=\left\{m \in \mathbb{N}: \prod_{i=1}^{m}\left|w_{i}\right|>M\right\}$. Let $M>0$ and $j \in \mathbb{N}$ such that $2^{j-1}<M \leq 2^{j}$, then $\cup_{n \geq j+1}\left\{2^{2 n}+j+1, \ldots, 2^{2 n}+2 n\right\} \subseteq A_{M}$. Obviously $B_{w}$ is weakly mixing since there exists an increasing sequence $\left(n_{k}\right)_{k}$ such that $\lim _{k \rightarrow \infty} \prod_{i=1}^{n_{k}} w_{i}=\infty$, see Chapter 4 (24). On the other hand, by Corollary $4.4(16),\left(B_{w}, B_{w}^{2}\right)$ is $d$-hypercyclic if and only if

$$
\bigcap_{t=1,2}\left\{m \in \mathbb{N}: t m \in A_{M}\right\} \in \mathcal{J}
$$

for any $M>0$. Now, take into consideration that $d$-hypercyclicity and $d$-topologically transitivity coincides for tuples of powers of a fixed unilateral weighted shift. Observe that $A_{1}=\cup_{n \in \mathbb{N}}\left\{2^{2 n}+1, \ldots, 2^{2 n}+2 n\right\}$ and $\{(m, 2 m): m \in \mathbb{N}\} \cap\left(A_{1} \times A_{1}\right)=\emptyset$. Hence $\left(B_{w}, B_{w}^{2}\right)$ is not $d$-topologically transitive.

On the other hand, the proof of Corollary 77 tell us that for any shift invariant family $\mathscr{F}$ (not necessarily a filter), satisfying both conditions $A_{M} \in \mathscr{F}$ and $\left\{m \in \mathbb{N}: 2 m \in A_{M}\right\} \in \mathscr{F}$, for any $M>0$ implies that the tuple $\left(B_{w}, B_{w}^{2}\right)$ satisfies the $d-\mathscr{F}$ Hypercyclicity Criterion with respect to $(k)$. In our case, $A_{M}$ is thick and

$$
\begin{array}{r}
\bigcup_{n \geq j+1}\left\{2^{2 n-1}+\left\lceil\frac{j+1}{2}\right\rceil, 2^{2 n-1}+\left\lceil\frac{j+1}{2}\right\rceil+1, \ldots, 2^{2 n-1}+n\right\} \subseteq \\
\left\{m \in \mathbb{N}: 2 m \in A_{M}\right\}
\end{array}
$$

and clearly the left-hand side of the last inclusion is a thick set.

\subsection{An $\mathscr{F}$-operator $T$ for which $\left(T, T^{2}\right)$ is not $d$ - $\mathscr{F}$}

In Theorem 3.8 (14) the authors show an example of a mixing Hilbert space operator $T$ such that $\left(T, T^{2}\right)$ is not $d$-mixing. A slight modification in the proof allows us to exhibit an operator with more detailed features, i.e. 


\section{DISJOINT HYPERCYCLICITY ALONG FILTERS}

a mixing Hilbert space operator $T$ such that $\left(T, T^{2}\right)$ is not $d$-syndetic, so in particular it is not $d$-mixing. As a consequence we have that Theorem 74 (respectively, Theorem 78) fails beyond the weighted shift setting, in other words, there exists a Hilbert space $\mathscr{F}$-operator $T$ such that $\left(T, T^{2}\right)$ is not $d$ - $\mathscr{F}$, for $\mathscr{F}=\Delta^{*}, \mathcal{J P}^{*}, \mathcal{P S}^{*}, \mathcal{S}$. We describe here all the details for the sake of completeness.

Let $1 \leq p<\infty,-\infty<a<b<+\infty$ and $k \in \mathbb{N}$. Recall that the Sobolev space $W^{k, p}[a, b]$ is the space of functions $f \in C^{k-1}[a, b]$ such that $f^{(k-1)}$ is absolutely continuous and $f^{(k)} \in L^{p}[a, b]$. The space $W^{k, p}[a, b]$ endowed with the norm

$$
\|f\|_{W^{k, p}[a, b]}=\left(\int_{a}^{b}\left(\sum_{j=0}^{k}\left|f^{(j)}(x)\right|^{p}\right) d x\right)^{1 / p}
$$

is a Banach space isomorphic to $L^{p}[0,1]$. Now, $W^{k, 2}[a, b]$ is a separable infinite-dimensional Hilbert space for each $k \in \mathbb{N}$. The family of operators to be considered lives on separable complex Hilbert spaces and is built from a single operator. Let $M \in \mathcal{L}\left(W^{2,2}[-\pi, \pi]\right)$ be defined by the formula

$$
M: W^{2,2}[-\pi, \pi] \longrightarrow W^{2,2}[-\pi, \pi], \quad M f(x)=\exp ^{i x} f(x) .
$$

Denote $\mathscr{H}=W^{2,2}[-\pi, \pi]$ and $M^{*}$ the dual operator. Then, $M^{*} \in \mathcal{L}\left(\mathscr{H}^{*}\right)$. For each $t \in[-\pi, \pi], \delta_{t} \in \mathscr{H}^{*}$, where $\delta_{t}: \mathscr{H} \longrightarrow \mathbb{C}, \delta_{t}(f)=f(t)$. Furthermore, the map $t \rightarrow \delta_{t}$ from $[-\pi, \pi]$ to $\mathscr{H}^{*}$ is norm-continuous. For a non-empty compact subset $K$ of $[-\pi, \pi]$, denote

$$
X_{K}=\overline{\operatorname{span}}\left\{\delta_{t}: t \in K\right\}
$$

where the closure of $\operatorname{span}\left\{\delta_{t}: t \in K\right\}$ is taken with respect to the norm of $\mathscr{H}^{*}$. 
Now, the functionals $\delta_{t}$ are linearly independent, $X_{K}$ is always a separable Hilbert space and $X_{K}$ is infinite dimensional if and only if $K$ is infinite. The following condition holds

$$
M^{*} \delta_{t}=\exp ^{i t} \delta_{t}, \quad \text { for } \quad \text { each } t \in[-\pi, \pi]
$$

Hence, each $X_{K}$ is an invariant subspace for $M^{*}$, which allows us to consider the operator

$$
Q_{K} \in \mathcal{L}\left(X_{K}\right), \quad Q_{K}=\left.M^{*}\right|_{X_{K}} .
$$

The following is taken from (14) which tells us when $Q_{K}$ is mixing or transitive, we omit the proof.

Proposition 84 ((14), Proposition 3.9). Let $K$ a non-empty compact subset of $[-\pi, \pi]$. If $K$ has no isolated points, then $Q_{K}$ is mixing. If $K$ has an isolated point, the $Q_{K}$ is non-transitive.

Hence, in order to obtain a mixing operator $T$ such that $\left(T, T^{2}\right)$ is not $d$-syndetic, it will be enough to find a non-empty compact set $K \subset[-\pi, \pi]$ with no isolated points such that the sequence $\left(2 Q_{K}^{n}-Q_{K}^{2 n}\right)_{n}$ is not a syndetic operator. As in the proof of theorem 3.8 (14) we need lemma A.3 (14).

Lemma 85 ((14), Lemma A.3). There exists a sequence $\left(f_{n}\right)_{n \in \mathbb{N}}$ of $2 \pi$ periodic functions on $\mathbb{R}$ such that $\left.f_{n}\right|_{[-\pi, \pi]} \in W^{2,2}[-\pi, \pi]$, the sequence $\left(\left\|f_{n}\right\|_{W^{2,2}[-\pi, \pi]}\right)_{n}$ is bounded and $f_{n}(x)=2 \exp ^{i n x}-\exp ^{2 i n x}$ whenever $\left|x-\frac{2 \pi m}{n}\right| \leq$ $2 / n^{5}$, for some $m \in \mathbb{Z}$.

Now, consider the set

$$
K=\left\{\sum_{n=1}^{\infty} \sum_{r=0}^{n} 2 \pi \epsilon_{n, r} \cdot \frac{1}{2^{7^{n}}+r}: \epsilon \in\{0,1\}^{\mathbb{N} \times \mathbb{N}}\right\} .
$$




\section{DISJOINT HYPERCYCLICITY ALONG FILTERS}

Note that $K$ is a compact subset of $[-\pi, \pi]$ with no isolated points and by proposition 84. $Q_{K} \in \mathcal{L}\left(X_{K}\right)$ is a mixing operator. We adapt the proof of [ (14), Proposition 3.10] to the compact set $K$ defined in 4.2 .2 , this is the content of the following:

Proposition 86. Let $K$ be the compact subset of $[-\pi, \pi]$ defined in (4.2.2), then the sequence $\left(2 Q_{K}^{k_{n, r}}-Q_{K}^{2 k_{n, r}}\right)_{n \in \mathbb{N}, 0 \leq r \leq n}$ of continuous linear operators on $X_{K}$ is non-transitive, where $k_{n, r}=2^{7^{n}}+r$ with $0 \leq r \leq n, n \in \mathbb{N}$.

Proof. For $f \in W^{2,2}[-\pi, \pi]$, consider $\phi_{f} \in X_{K}^{*}$ defined by $\phi_{f}(y)=y(f)$. Remark that $\left\|\phi_{f}\right\| \leq\|f\|_{W^{2,2}[-\pi, \pi]}$ and that $\phi_{f}=\phi_{g}$ if $\left.f\right|_{K}=\left.g\right|_{K}$, then

$$
\left\|\phi_{f}\right\| \leq \inf \left\{\|g\|_{W^{2,2}[-\pi, \pi]}:\left.f\right|_{K}=\left.g\right|_{K}\right\}
$$

Use the symbol 1 to denote the constant 1 function in $W^{2,2}[-\pi, \pi]$. The functional $\phi_{\mathbf{1}} \in X_{K}^{*}$ is non-zero, since $\phi_{\mathbf{1}}\left(\delta_{t}\right)=1$ for each $t \in K$. Denote $T_{n, r}=2 Q_{K}^{k_{n, r}}-Q_{K}^{2 k_{n, r}} \in \mathcal{L}\left(X_{K}\right)$ and estimate $\left\|T_{n, r}^{*}\right\| \phi_{\mathbf{1}}$. By definition of $Q_{K}$, it holds $Q_{K}^{*} \phi_{f}=\phi_{M f}$, for each $f \in W^{2,2}[-\pi, \pi]$, where $M$ is the multiplication operator defined in 4.2.1). It follows that $T_{n, r}^{*} \phi_{\mathbf{1}}=\phi_{h_{k_{n, r}}}$, where $h_{j}(t)=2 \exp ^{i j t}-\exp ^{2 i j t}$. By lemma 85 , there is a bounded sequence $\left(f_{k_{n, r}}\right)_{n \in \mathbb{N}, 0 \leq r \leq n}$ in the Hilbert space $W^{2,2}[-\pi, \pi]$ such that $f_{k_{n, r}}(t)=h_{k_{n, r}}(t)$ whenever $\left|t-\frac{2 \pi m}{k_{n, r}}\right| \leq \frac{2}{\left(k_{n, r}\right)^{5}}$ for some $m \in \mathbb{Z}$.

Now, let $t \in K$, then $t=\sum_{j=1}^{\infty} \sum_{r=0}^{j} \frac{2 \pi \epsilon_{j, r}}{k_{j, r}}$ for some $\epsilon \in\{0,1\}^{\mathbb{N} \times \mathbb{N}}$. For each $n \in \mathbb{N}$, it holds $t=y+u$, where $y=\sum_{j=1}^{n+1} \sum_{r=0}^{j} \frac{2 \pi \epsilon_{j, r}}{k_{j, r}}$ and $u=$ $\sum_{j=n+2}^{\infty} \sum_{r=0}^{j} \frac{2 \pi \epsilon_{j, r}}{k_{j, r}}$. Obviously there exists $m \in \mathbb{N}$ such that $y \leq 2 \pi m / k_{n, r}$ 
and

$$
\begin{array}{r}
0 \leq u \leq 2 \pi \sum_{j=n+2}^{\infty} \sum_{r=0}^{j} \frac{1}{k_{j, r}} \\
=2 \pi\left[\left(\frac{1}{2^{7^{n+2}}}+\frac{1}{2^{7^{n+2}}+1}+\cdots+\frac{1}{2^{7^{n+2}+n+2}}\right)\right. \\
\left.+\left(\frac{1}{2^{7^{n+3}}}+\frac{1}{2^{7^{n+3}}+1}+\cdots+\frac{1}{2^{7^{n+3}}+n+3}\right)+\ldots\right] \\
<2 \pi\left[\left(\frac{1}{2^{7^{n+1}}}+\frac{1}{2^{7^{n+1}+1}}+\cdots+\frac{1}{2^{7^{n+1}+n+2}}\right)\right. \\
+\left(\frac{1}{2^{7^{n+2}}}+\frac{1}{\left.\left.2^{7^{n+2}+1}+\cdots+\frac{1}{2^{7^{n+2}+n+3}}\right)+\ldots\right]}\right. \\
<2 \pi \sum_{j=7^{n+1} 2^{-j}<\frac{4 \pi}{2^{7^{n+1}}}}=\frac{4 \pi}{\left(2^{7^{n}}\right)^{7}}<\frac{4 \pi}{\left(2^{7^{n}}+r\right)^{6}}<\frac{2}{\left(2^{7^{n}}+r\right)^{5}}=2 k_{n, r}^{-5} .
\end{array}
$$

Hence, $t=u+y \leq u+\left(2 \pi m / k_{n, r}\right)$, which implies $\left|t-\left(2 \pi m / k_{n, r}\right)\right| \leq 2 k_{n, r}^{-5}$. Thus $f_{k_{n, r}}(t)=h_{k_{n, r}}(t)$ for each $t \in K$ and $n \in \mathbb{N}$. By 4.2.3, $\left\|\phi_{k_{n, r}}\right\| \leq$ $\left\|f_{k_{n, r}}\right\|_{W^{2,2}[-\pi, \pi]}$ for $n \in \mathbb{N}, 0 \leq r \leq n$. Since the sequence $\left(f_{k_{n, r}}\right)_{n, r}$ is bounded in $W^{2,2}[-\pi, \pi]$, the sequence $\left(\left\|\phi_{h_{k_{n, r}}}\right\|\right)_{n, r}$ is bounded, i.e. there exists $C>0$ such that $\left\|\phi_{h_{k_{n, r}}}\right\| \leq C$ for each $n \in \mathbb{N}, 0 \leq r \leq n$. Since $T_{n, r}^{*} \phi_{\mathbf{1}}=\phi_{h_{k_{n, r}}}$, it follows that $\left|\phi_{\mathbf{1}}\left(T_{n, r}(x)\right)\right|=\left|T_{n, r}^{*} \phi_{\mathbf{1}}(x)\right| \leq C\|x\|$ for each $x \in X_{K}$. Since $\phi_{1}$ is a non-zero continuous linear functional on $X_{K}$, by Hahn-Banach theorem the set $\left\{T_{n, r} x: n \in \mathbb{N}, 0 \leq r \leq n\right\}$ can not be dense in $X_{K}$, for any given $x \in X_{K}$. In other words, $\left\{T_{n, r}: n \in \mathbb{N}, 0 \leq r \leq n\right\}$ is non-universal.

Now, we can prove the main result of this section:

Theorem 87. There exists $T \in \mathcal{L}\left(l_{2}\right)$ such that $T$ is mixing and the sequence of operators $\left(2 T^{n}-T^{2 n}\right)_{n \in \mathbb{N}}$ is not $\mathcal{S}$-transitive. In particular, $\left(T, T^{2}\right)$ is not d-syndetic. Consequently, $T$ is $\mathscr{F}$-operator but $\left(T, T^{2}\right)$ is not d-Fु, where $\mathscr{F}=\Delta^{*}, \mathcal{J P}^{*}, \mathcal{P S}^{*}, \mathcal{S}$. 


\section{DISJOINT HYPERCYCLICITY ALONG FILTERS}

Proof. Let $K$ the compact set defined in 4.2.2. By proposition $84, Q_{K}$ is a mixing operator on the separable infinite dimensional Hilbert space $X_{K}$. On the other hand, by proposition $86 .\left(2 Q_{K}^{a_{n}}-Q_{K}^{2 a_{n}}\right)_{n \in \mathbb{N}}$ is non-transitive for some thick set $A$ written increasingly as $A=\left(a_{n}\right)_{n}$. Hence, there exists opene sets $U, V$ in $X_{K}$ such that $\left(2 Q_{K}^{a_{n}}-Q_{K}^{2 a_{n}}\right)(U) \cap V=\emptyset$, for any $n \in \mathbb{N}$. In other words,

$$
\left\{n \in \mathbb{N}:\left(2 Q_{K}^{n}-Q_{K}^{2 n}\right)(U) \cap V \neq \emptyset\right\} \cap A=\emptyset,
$$

i.e., $\left(2 Q_{K}^{n}-Q_{K}^{2 n}\right)_{n \in \mathbb{N}}$ is not a $\mathcal{S}$-transitive sequence of operators. In particular, $\left(Q_{K}, Q_{K}^{2}\right)$ is not $d$-syndetic. In fact, let $U, V$ opene sets in $X_{K}$ such that $\left\{n \in \mathbb{N}:\left(2 Q_{K}^{n}-Q_{K}^{2 n}\right)(U) \cap V \neq \emptyset\right\}$ is not a syndetic set, and pick $V_{0}$ opene set such that $2 V_{0}-V_{0} \subseteq V($ denote $B(x ; r)$ the open ball centered at $x$ in $X_{K}$ with radius r. Pick $x \in X_{K}, r \in \mathbb{R}_{+}$such that $B(x ; r) \subset V$, then set $\left.V_{0}:=B(x ; r / 3)\right)$. Hence,

$\left\{n \in \mathbb{N}: U \cap Q_{K}^{-n}\left(V_{0}\right) \cap Q_{K}^{-2 n}\left(V_{0}\right) \neq \emptyset\right\} \subseteq\left\{n \in \mathbb{N}:\left(2 Q_{K}^{n}-Q_{K}^{2 n}\right)(U) \cap V \neq \emptyset\right\}$. Consequently, $\left\{n \in \mathbb{N}: U \cap Q_{K}^{-n}\left(V_{0}\right) \cap Q_{K}^{-2 n}\left(V_{0}\right) \neq \emptyset\right\}$ can not be a syndetic set and then $\left(Q_{K}, Q_{K}^{2}\right)$ is not $d$-syndetic. Since all separable infinite dimensional Hilbert spaces are isomorphic to $l_{2}$, there is a mixing $T \in \mathcal{L}\left(l_{2}\right)$ such that the sequence $\left(2 T^{n}-T^{2 n}\right)_{n \in \mathbb{N}}$ is not $\mathcal{S}$-transitive, and then $\left(T, T^{2}\right)$ is not $d$-syndetic.

\subsection{Reiteratively hypercyclicity vs. $d-\mathscr{F}$ tuples}

In this section we will examine the relationship between reiterative hypercyclicity and $d-\mathscr{F}$ tuples.

On one hand, by Proposition 42 there exists an $\mathcal{J P}^{*}$-weighted backward shift which is not reiteratively hypercyclic on both $c_{0}\left(\mathbb{Z}_{+}\right)$and $l_{p}\left(\mathbb{Z}_{+}\right)(1 \leq$ $p<\infty)$. As a consequence, by Theorem 78 we have the following: 
Proposition 88. There exists a weighted backward shift $B_{w}$ on both $c_{0}\left(\mathbb{Z}_{+}\right)$ and $l_{p}\left(\mathbb{Z}_{+}\right),(1 \leq p<\infty)$, such that the tuple $\left(B_{w}, \ldots, B_{w}^{r}\right)$ is $d$-JP* for any $r \in \mathbb{N}$ and $B_{w}$ is not reiteratively hypercyclic.

Conversely, by Proposition 38 (respectively, Proposition 41), any reiteratively hypercyclic weighted backward shift on $c_{0}$ or $l_{p},(1 \leq p<\infty)$ is necessarily a $\Delta^{*}$-operator. On the other hand, by Proposition 56 (respectively, Proposition 57), any reiteratively hypercyclic weighted shift $B_{w}$ on $X=c_{0}$ or $l_{p},(1 \leq p<\infty)$ satisfies $B_{w} \oplus \cdots \oplus B_{w}^{r}$ is $\mathcal{E}^{*}$-operator in $X^{r}$, for any $r \in \mathbb{N}$ (recall that the filter $\mathcal{E}^{*}$ satisfies: $\mathcal{J P}^{*} \varsubsetneqq \mathcal{E}^{*} \varsubsetneqq \mathcal{S}$ ). Then, by Corollary 72 (respectively, Corollary 77 ) we have that $\left(B_{w}, \ldots, B_{w}^{r}\right)$ is $d$ - $\mathcal{E}^{*}$, for any $r \in \mathbb{N}$. Now, by Theorem 74 (respectively, Theorem 78) we can unify all of these conclusions by stating:

Theorem 89. Let $X=c_{0}(\mathbb{Z})$ or $l_{p}(\mathbb{Z}),(1 \leq p<\infty)$. If $B_{w}$ is a reiteratively hypercyclic bilateral weighted backward shift on $X$ then $\left(B_{w}, \ldots, B_{w}^{r}\right)$ is $d$ $\Delta^{*}$, for any $r \in \mathbb{N}$.

Analogously,

Theorem 90. Let $X=c_{0}\left(\mathbb{Z}_{+}\right)$or $l_{p}\left(\mathbb{Z}_{+}\right),(1 \leq p<\infty)$. If $B_{w}$ is a reiteratively hypercyclic unilateral weighted backward shift on $X$ then $\left(B_{w}, \ldots, B_{w}^{r}\right)$ is $d-\Delta^{*}$, for any $r \in \mathbb{N}$.

Observe that the conclusion of Theorem 90 is optimal since in (3) appears an example of a frequently hypercyclic, hence reiteratively hypercyclic weighted shift on $c_{0}\left(\mathbb{Z}_{+}\right)$which is not mixing.

In general, for linear operators, we obtain the following theorem: 


\section{DISJOINT HYPERCYCLICITY ALONG FILTERS}

Theorem 91. Let $T$ bounded and linear operator on a topological vector space $X$. If $T$ is reiteratively hypercyclic then $\left(T, \ldots, T^{r}\right)$ is d-syndetic or not d-transitive, for any $r \in \mathbb{N}$.

The main ingredient of the proof is a result of Bergelson and McCutcheon concerning essential idempotents of $\beta \mathbb{N}$, and Szemerédi's theorem for generalized polynomials (10).

Proof. Fix $r \in \mathbb{N}$. Let $T$ reiteratively hypercyclic, then there exists $x \in X$ such that

$$
N_{T}(\underbrace{U, \ldots, U}_{r} ; U) \in \mathcal{E}^{*},
$$

for any opene set $U$ in $X$, by Theorem 63

Next, let $\left(U_{j}\right)_{j=0}^{r}$ a finite sequence of opene sets in $X$. Now, suppose that $\left(T, \ldots, T^{r}\right)$ is $d$-transitive, we must show that $N_{T}\left(U_{1}, \ldots, U_{r} ; U_{0}\right) \in \mathcal{S}$. In fact, there exists $n \in \mathbb{N}$ such that

$$
V_{n}:=T^{-n} U_{1} \cap \cdots \cap T^{-r n} U_{r} \cap U_{0} \neq \emptyset .
$$

Thus, $V_{n}$ is open, then pick $O_{1}, O_{2}$ opene sets such that $O_{1}+O_{2} \subset V_{n}$, then

$$
T^{j n}\left(O_{1}+O_{2}\right) \subset U_{j}, \quad \text { for any } j \in\{0, \ldots, r\} .
$$

Now, by (4.3.1) since $\mathcal{E}^{*}$ is a filter we have

$$
A:=N_{T}(\underbrace{O_{1}, \ldots, O_{1}}_{r} ; O_{1}) \cap N_{T}(\underbrace{O_{2}, \ldots, O_{2}}_{r} ; O_{2}) \in \mathcal{E}^{*} \varsubsetneqq \mathcal{S} .
$$

Let us show that $A+n \subseteq N\left(U_{1}, \ldots, U_{r} ; U_{0}\right)$, then we are done because $A+n \in \mathcal{S}$ since $\mathcal{S}$ is shift invariant. 
In fact, let $t \in A+n$, then $t-n \in A$, which means

$$
\begin{gathered}
T^{-t} T^{n}\left(O_{1}\right) \cap \cdots \cap T^{-r t} T^{r n}\left(O_{1}\right) \cap O_{1} \neq \emptyset \\
T^{-t} T^{n}\left(O_{2}\right) \cap \cdots \cap T^{-r t} T^{r n}\left(O_{2}\right) \cap O_{2} \neq \emptyset .
\end{gathered}
$$

By the linearity of $T$ we obtain

$$
T^{-t}\left(T^{n}\left(O_{1}+O_{2}\right)\right) \cap \cdots \cap T^{-r t}\left(T^{r n}\left(O_{1}+O_{2}\right)\right) \cap\left(O_{1}+O_{2}\right) \neq \emptyset .
$$

Then we conclude by 4.3.2, i.e.

$$
T^{-t} U_{1} \cap \cdots \cap T^{-r t} U_{r} \cap U_{0} \neq \emptyset
$$

Corollary 92. The operator $Q_{K}$ given by Theorem 87, is a (non-weighted shift) mixing operator which is not reiteratively hypercyclic.

Corollary 92 follows by Theorems 87 and 91 .

\subsection{Moving from syndetic to reiteratively hyper- cyclic weighted shifts on $c_{0}\left(\mathbb{Z}_{+}\right)$}

Recall that reiteratively hypercyclic operators are syndetic (Proposition 37) but the converse is not true (Proposition 42). In this section, we will discuss under which conditions we can obtain reiterative hypercyclicity from syndeticity, for weighted backward shifts on $X=c_{0}\left(\mathbb{Z}_{+}\right)$or $l^{p}\left(\mathbb{Z}_{+}\right),(1 \leq p<$ $\infty)$.

Consider a sequence $\left(H_{m}\right)_{m}$ of pairwise disjoint subsets of $\mathbb{N}$ such that

$$
\left(H_{m}+[0, m]\right) \cap H_{n}=\emptyset \quad \forall m \neq n .
$$




\section{DISJOINT HYPERCYCLICITY ALONG FILTERS}

Suppose that each $H_{m}$ is partitioned into a sequence of pairwise disjoint finite sets $\left(H_{k, m}\right)_{k}$ such that for any $k_{1}<k_{2}$

$$
s<t \quad \forall(s, t) \in H_{k_{1}, m} \times H_{k_{2}, m} .
$$

Denote $\mathcal{H}:=\left\{\left(H_{k, m}\right)_{k}: m \in \mathbb{N}\right\}$. Let $l \in H_{m}$, then there exists a unique $k$ such that $l \in H_{k, m}$. Set $[l]_{H_{m}}:=H_{k, m}$. Fix $k_{0}, m_{0}$ and denote

$$
\widetilde{H}_{k_{0}, m_{0}}^{(m)}:=\left[\min \left(\left(\max H_{k_{0}, m_{0}}, \infty\right) \cap H_{m}\right)\right]_{H_{m}} .
$$

Let $\mathscr{F}$ be a family in $\mathbb{N}$ and $\mathbf{w}=\left(w_{n}\right)_{n}$ a bounded weight.

Definition 93. We say that a triple $\left(\mathbf{w},\left(H_{m}\right)_{m}, \mathcal{H}\right)$

- is called $\mathscr{F}$-triple if $H_{m} \in \mathscr{F}$ for any $m \in \mathbb{N}$.

- satisfies property $\mathscr{P}$ if

$$
w_{1+r} \cdots w_{(s+r)-t}>m \cdot n
$$

for any $(s, t) \in \widetilde{[t]}]_{H_{n}}^{(m)} \times H_{n}, m \neq n, r \in\{0 \ldots m\}$, and

$$
w_{1+r} \cdots w_{(s+r)-t}>m^{2}
$$

for any $(s, t) \in H_{k, m} \times H_{k, m}, k, m \in \mathbb{N}, s>t, r \in\{0 \ldots m\}$.

Let us recall a characterization of piecewise syndeticity for sets, see (27). A set $A \subset \mathbb{N}$ is piecewise syndetic $(A \in \mathcal{P S})$ if there exists a natural number $G(A)$ such that $\cup_{t=1}^{G(A)}(-t+A)$ is a thick set.

Hence, $A \in \mathcal{P S}$ is equivalent to say:

$$
\forall L>0, \exists x: x, \ldots, x+L \in \cup_{t=1}^{G(A)}(-t+A)
$$


i.e.

$$
\forall L>0, \exists x:(x+i)+t_{i} \in A
$$

for some $1 \leq t_{i} \leq G(A)$ and any $0 \leq i \leq L$. Recall that $\overline{\mathcal{B D}}=\{A \subseteq \mathbb{N}$ : $\overline{B d}(A)>0\}$.

Proposition 94. Let $B_{w}$ a syndetic weighted backward shift, then there exists a $\overline{\mathcal{B D}}$-triple associated to $w$.

Proof. Recall that each central set is piecewise syndetic. On the other hand, as a consequence of $[(26)$, Theorem 2.12] it holds that any central subset of $\mathbb{N}$ can be partitioned into infinitely many pairwise disjoint central sets, see [(26), Corollary 2.13]. Hence, we can apply this theorem as many times as necessary, taking $\mathbb{N}$ as the starting set, in order to obtain a sequence $\left(D_{m}\right)_{m}$ of pairwise disjoint $\mathcal{P S}$-sets satisfying

$$
\left(D_{m}+[0, m]\right) \cap D_{n}=\emptyset
$$

for any $m \neq n$.

We will define a sequence of natural numbers $\left(l_{j, k, m}\right)_{\substack{m, k \in \mathbb{N} \\ 1 \leq j \leq k}}$ and set

$$
H_{m}:=\left\{l_{j, k, m}: k \in \mathbb{N}, 1 \leq j \leq k\right\}
$$

and

$$
H_{k, m}:=\left\{l_{j, k, m}: 1 \leq j \leq k\right\} .
$$

We begin by defining $H_{1}=\left\{l_{j, k, 1}: k \in \mathbb{N}, 1 \leq j \leq k\right\}, H_{2}$ and so on as $m$ increases, such that $\min H_{m} \geq m+G\left(D_{m}\right)$, for any $m \in \mathbb{N}$. Now, at each level $m$, we will proceed by blocks $H_{k, m}=\left\{l_{j, k, m}: 1 \leq j \leq k\right\}$ indexed by $k$, as $k$ increases such that

$$
l_{j, k, m}+m+t_{j}=l_{j+1, k, m}
$$




\section{DISJOINT HYPERCYCLICITY ALONG FILTERS}

for some $1 \leq t_{j} \leq G\left(D_{m}\right)$ and any $1 \leq j \leq k$ and

$$
l_{k-1, k-1, m}<l_{1, k, m}<l_{k, k, m}<l_{1, k+1, m}
$$

for any $k, m \in \mathbb{N}$.

Before beginning the construction of the sequence $\left(H_{m}\right)_{m}$, let us recall the following. For any $M>0, j \in \mathbb{N}$, denote $A_{M ; j}=\left\{n \in \mathbb{N}: \prod_{i=1}^{n}\left|w_{i+j}\right|>\right.$ $M\}$. By hypothesis $B_{w}$ is syndetic backward shift, which is equivalent to say $\cap_{j \in F} A_{M ; j} \in \mathcal{P S}^{*}$ for any $M>0$ and any finite subset of natural numbers $F$, according to Proposition 22 and Proposition 28, Furthermore, recall that any $\mathcal{P S}^{*}$-set $A$ is thickly syndetic, i.e. for any $L>0$, there exists a syndetic sequence $\left(x_{n}\right)_{n}$ such that $\left\{x_{n}, x_{n}+1, \ldots, x_{n}+L: n \in \mathbb{N}\right\} \subseteq A$.

Now, how to proceed? Suppose we have defined $H_{\hat{m}}$ for any $\hat{m}<m$. Set $l_{1,1, m}=\max \left(m+G\left(D_{m}\right), \min _{l \in D_{m}} l\right)$. Let $k \in \mathbb{N}$ and suppose we have defined $\left\{l_{j, \hat{k}, m}: \hat{k}<k, 1 \leq j \leq \hat{k}\right\}$. Let us define the block $H_{k, m}=\left\{l_{j, k, m}\right.$ : $1 \leq j \leq k\}$.

By hypothesis, the set $A^{(k, m)}:=\cap_{r=1}^{m} A_{m \cdot l_{k-1, k-1, m} ; r}$ is thickly syndetic, hence there exists some syndetic sequence $\left(x_{n}^{(k, m)}\right)_{n}$ such that

$$
x_{n}^{(k, m)}, \ldots, x_{n}^{(k, m)}+l_{k-1, k-1, m}, \ldots, x_{n}^{(k, m)}+3 l_{k-1, k-1, m} \subseteq A^{(k, m)}
$$

for any $n \in \mathbb{N}$. By commodity, set $D_{m}-l_{k-1, k-1, m}$ instead of $\left(D_{m}-\right.$ $\left.l_{k-1, k-1, m}\right) \cap \mathbb{N}$, then because $\mathcal{P S}$ is shift invariant, we have $D_{m}-l_{k-1, k-1, m} \in$ $\mathcal{P S}$ with $G\left(D_{m}\right)=G\left(D_{m}-l_{k-1, k-1, m}\right)$. Let $S^{(k, m)}$ the gap of $\left(x_{n}^{(k, m)}\right)_{n}$, then there exists $x \in \mathbb{N}$ such that

$$
\begin{gathered}
x+t_{0} \in D_{m}-l_{k-1, k-1, m} \\
(x+1)+t_{1} \in D_{m}-l_{k-1, k-1, m} \\
\ldots \\
x+S^{(k, m)}+k\left(m+G\left(D_{m}\right)\right)+t_{S^{(k, m)}+k\left(m+G\left(D_{m}\right)\right)} \in D_{m}-l_{k-1, k-1, m}
\end{gathered}
$$


for some $1 \leq t_{i} \leq G\left(D_{m}\right)$ and any $0 \leq i \leq S^{(k, m)}+k\left(m+G\left(D_{m}\right)\right)$.

Hence, there exists $0 \leq S \leq S^{(k, m)}$ and $n \in \mathbb{N}$ such that $x_{n}^{(k, m)}=x+S$. Then set

$$
\begin{gathered}
l_{1, k, m}:=x_{n}^{(k, m)}+l_{k-1, k-1, m}+s_{1} \in D_{m} \\
l_{2, k, m}:=l_{1, k, m}+m+s_{2} \in D_{m} \\
\ldots \\
l_{k, k, m}:=l_{k-1, k, m}+m+s_{k} \in D_{m}
\end{gathered}
$$

for some $1 \leq s_{i} \leq G\left(D_{m}\right)$ and any $1 \leq i \leq k$. Observe that automatically it holds $l_{k-1, k-1, m}<l_{1, k, m}<l_{k, k, m}$. On the other hand, by 4.4.2 and since $k\left(m+G\left(D_{m}\right)\right)<2 l_{k-1, k-1, m}$, for any $k \geq 2$ (recall that $l_{1,1, m} \geq$ $\left.m+G\left(D_{m}\right)\right)$, we have the following

$$
\begin{gathered}
{\left[l_{1, k, m}, l_{1, k, m}+m\right] \subseteq A^{(k, m)}} \\
{\left[l_{2, k, m}, l_{2, k, m}+m\right] \subseteq A^{(k, m)}} \\
\cdots \\
{\left[l_{k, k, m}, l_{k, k, m}+m\right] \subseteq A^{(k, m)} .}
\end{gathered}
$$

Again by 4.4.2

$$
l_{j, k, m}-l \in A^{(k, m)}, \quad \forall l \leq l_{k-1, k-1, m} \quad 1 \leq j \leq k .
$$

Note that the intervals $(l+[0, m])_{\substack{l \in H_{m} \\ m \in \mathbb{N}}}$ are actually disjoint by condition 4.4.1. Finally let us see that $H_{m}$ has positive upper Banach density for any $m \in \mathbb{N}$. Let $m \in \mathbb{N}$, note that

$$
\alpha^{n}:=\underset{k}{\limsup }\left|H_{m} \cap\left[l_{1, k, m}, l_{1, k, m}+n\left(m+G\left(D_{m}\right)\right)\right]\right| \geq n+1 .
$$




\section{DISJOINT HYPERCYCLICITY ALONG FILTERS}

Hence,

$\overline{B d}\left(H_{m}\right) \geq \lim _{n} \frac{\alpha^{n}}{n\left(m+G\left(D_{m}\right)\right)} \geq \lim _{n} \frac{n+1}{n\left(m+G\left(D_{m}\right)\right)}=\frac{1}{m+G\left(D_{m}\right)}>0$.

So, we can associate to the weight of a syndetic weighted shift a $\overline{\mathcal{B D}}$ triple with further properties enumerated in the above construction.

Let $B_{w}$ a syndetic weighted backward shift, any $\overline{\mathcal{B D}}$-triple with the characteristics described in the proof of Proposition 94, will be called a $\overline{\mathcal{B D}}$-triple associated to $w$. Clearly, by construction a $\overline{\mathcal{B D}}$-triple associated to $w$ is not unique.

Theorem 95. $B_{w}$ is reiteratively hypercyclic on $c_{0}\left(\mathbb{Z}_{+}\right)$if and only if $B_{w}$ is syndetic on $c_{0}\left(\mathbb{Z}_{+}\right)$provided there exists a $\overline{\mathcal{B D}}$-triple associated to $w$ satisfying property $\mathscr{P}$.

Proof. Set $X=c_{0}\left(\mathbb{Z}_{+}\right)$, we need to show that $B_{w}$ is reiteratively hypercyclic on $X$ provided $B_{w}$ is syndetic on $X$ with a $\overline{\mathcal{B D}}$-triple associated to $w$ satisfying property $\mathscr{P}$.

Without loss of generality, consider a dense sequence $(z(m))_{m \in \mathbb{N}}$ on $X$, where

$$
z(m)=\left(z(m)_{0}, \ldots, z(m)_{m}, 0,0 \ldots\right)
$$

and satisfying $\left|z(m)_{j}\right| \leq m$, for every $j=0, \ldots, m$. Define

$$
U_{m}=\{x \in X:\|x-z(m)\|<1 / m\}, m \in \mathbb{N} .
$$

It suffices to find $y \in X$ such that $\overline{B d}\left(N\left(y, U_{m}\right)\right)>0$ for any $m \in \mathbb{N}$. Take the pairwise disjoint intervals in $\mathbb{N}$

$$
I_{j, k, m}=\left[l_{j, k, m} ; l_{j, k, m}+m\right]
$$


$k, m \in \mathbb{N}, 1 \leq j \leq k$ and set

$$
y:=\sum_{\substack{k, m \in \mathbb{N} \\ 1 \leq j \leq k}} \sum_{i \in I_{j, k, m}} y_{i} e_{i} .
$$

Then, for any $m \in \mathbb{N}$, it suffices to show that $B_{w}^{l_{j, k, m}} y \in U_{m}$, for any $k \in \mathbb{N}, 1 \leq j \leq k$, since $H_{m} \in \overline{\mathcal{B D}}$. Note that

$$
B_{w}^{l_{j, k, m}} y=\left(\prod_{i=1}^{l_{j, k, m}} w_{i} y_{l_{j, k, m}}, \ldots, \prod_{i=1}^{l_{j, k, m}} w_{i+m} y_{l_{j, k, m}+m}, \prod_{i=1}^{l_{j, k, m}} w_{i+m+1} y_{l_{j, k, m}+m+1}, \ldots\right) .
$$

In order to guarantee $B_{w}^{l_{j, k, m}} y \in U_{m}$, we set

$$
\prod_{i=1}^{l_{j, k, m}} w_{i+r} y_{l_{j, k, m}+r}=z(m)_{r}, \quad r=0, \ldots, m
$$

which forces to define

$$
y_{l_{j, k, m}+r}:=\frac{z(m)_{r}}{\prod_{i=1}^{l_{j, k, m}} w_{i+r}}, \quad r=0, \ldots, m .
$$

At this point, we have all we need in order to verify condition

$$
\left\|B_{w}^{l_{j, k, m}} y-z(m)\right\|<1 / m
$$

for any $k, m \in \mathbb{N}, 1 \leq j \leq k$.

Consider an arbitrary trio $j_{0}, k_{0}, m_{0}$.

$$
\begin{aligned}
& B_{w}^{l_{j_{0}, k_{0}, m_{0}}} y-z\left(m_{0}\right)=(\underbrace{0, \ldots, 0}_{\left(m_{0}+1\right)-\text { times }}, \prod_{i=1}^{l_{j_{0}, k_{0}, m_{0}}} w_{i+m_{0}+1} y_{l_{j_{0}, k_{0}, m_{0}}+m_{0}+1}, \ldots, \\
& \left.\ldots, \prod_{i=1}^{l_{j_{0}, k_{0}, m_{0}}} w_{i+t} y_{l_{j_{0}, k_{0}, m_{0}}+t}, \ldots\right) \text {. }
\end{aligned}
$$




\section{DISJOINT HYPERCYCLICITY ALONG FILTERS}

If $y_{l_{j_{0}, k_{0}, m_{0}}+t} \neq 0, m_{0}+1 \leq t$ there exists $k, m$ such that $l_{j_{0}, k_{0}, m_{0}}+t=$ $l_{j, k, m}+r$ with $1 \leq j \leq k, 0 \leq r \leq m$. Hence,

$$
y_{l_{j_{0}, k_{0}, m_{0}}+t}=y_{l_{j, k, m}+r}=\frac{z(m)_{r}}{\prod_{i=1}^{l_{j, k, m}} w_{i+r}}
$$

and

$$
\begin{gathered}
\prod_{i=1}^{l_{j_{0}, k_{0}, m_{0}}} w_{i+t} y_{l_{j_{0}, k_{0}, m_{0}}+t}=\frac{w_{1+l_{j, k, m}+r-l_{j_{0}, k_{0}, m_{0}}} \cdots w_{l_{j, k, m}+r}}{w_{1+r} \cdots w_{l_{j, k, m}+r}} z(m)_{r}= \\
=\frac{z(m)_{r}}{w_{1+r} \cdots w_{l_{j, k, m}+r-l_{j_{0}, k_{0}, m_{0}}}} .
\end{gathered}
$$

Let $m \neq m_{0}$, then there exists a unique number $\widetilde{k}(m)$ such that $l_{\widetilde{k}(m)-1, \widetilde{k}(m)-1, m}<$ $l_{j_{0}, k_{0}, m_{0}}<l_{1, \widetilde{k}(m), m}$. Note that $\left[\widetilde{l_{j_{0}, k_{0}, m_{0}}}\right]_{H_{m_{0}}}^{(m)}=\left[l_{1, \widetilde{k}(m), m}\right]_{H_{m}}$, then

$$
\begin{aligned}
& B_{w}^{l_{j_{0}, k_{0}, m_{0}}} y-z\left(m_{0}\right)=\sum_{\substack{k, m \in \mathbb{N} \\
l_{j_{0}, k_{0}, m_{0}<l_{j, k, m}}}} \sum_{j=1}^{k} \sum_{r=0}^{m} \frac{z(m)_{r}}{w_{1+r} \cdots w_{l_{j, k}, m}+r-l_{j_{0}, k_{0}, m_{0}}} e_{l_{j, k, m}+r}=
\end{aligned}
$$

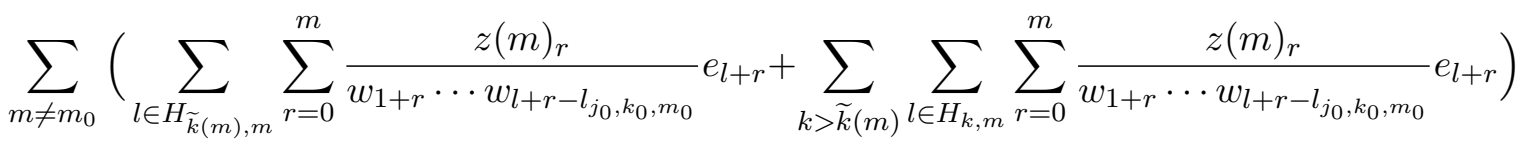

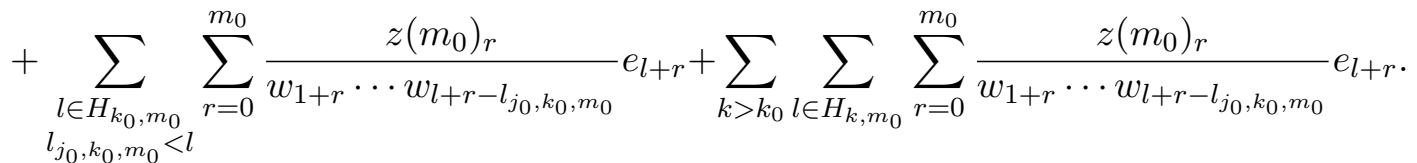

Now, let us estimate each summand. The fact that the $\overline{\mathcal{B D}}$-triple associated to $w$ satisfies property $\mathscr{P}$ implies

$$
\sup _{\substack{r \in\{0 \ldots m\} \\ l \in H_{\tilde{k}(m), m} \\ m \neq m_{0}}} \frac{\left|z(m)_{r}\right|}{\left|w_{1+r} \cdots w_{l+r-l_{j_{0}, k_{0}, m_{0}}}\right|}<\frac{m}{m \cdot m_{0}}=\frac{1}{m_{0}} .
$$


By 4.4.4 we have

$$
\begin{gathered}
\sup _{\substack{r \in\{0 \ldots m\} \\
l \in H_{k, m} \\
k>\widetilde{k}(m) \\
m \neq m_{0}}} \frac{\left|z(m)_{r}\right|}{\mid w_{1+r} \cdots w_{l+r-l_{j_{0}, k_{0}, m_{0}} \mid}}=\sup _{\substack{r \in\{0 \ldots m\} \\
j=\{1 \ldots k\} \\
k>\widetilde{k}(m) \\
m \neq m_{0}}} \frac{\left|z(m)_{r}\right|}{\mid w_{1+r} \cdots w_{l_{j, k, m}+r-l_{j_{0}, k_{0}, m_{0}} \mid}}< \\
<\frac{m}{m \cdot l_{k-1, k-1, m}}<\frac{1}{l_{j_{0}, k_{0}, m_{0}}}<\frac{1}{m_{0}} .
\end{gathered}
$$

Concerning the last two summands we have the following estimations

$$
\sup _{\substack{r \in\left\{0 \ldots m_{0}\right\} \\ l \in H_{k_{0}, m_{0}} \\ l_{j_{0}, k_{0}, m_{0}}<l}} \frac{\left|z\left(m_{0}\right)_{r}\right|}{\mid w_{1+r} \cdots w_{l+r-l_{j_{0}, k_{0}, m_{0}} \mid}}<\frac{m_{0}}{\left(m_{0}\right)^{2}}=\frac{1}{m_{0}}
$$

that follows because the $\overline{\mathcal{B D}}$-triple associated to $w$ satisfies property $\mathscr{P}$. Finally, again by 4.4.4

$$
\begin{gathered}
\sup _{\substack{r \in\left\{0 \ldots m_{0}\right\} \\
l \in H_{k, m_{0}} \\
k>k_{0}}} \frac{\left|z\left(m_{0}\right)_{r}\right|}{\left|w_{1+r} \cdots w_{l+r-l_{j_{0}, k_{0}, m_{0}} \mid}\right|}=\sup _{\substack{r \in\left\{0 \ldots m_{0}\right\} \\
j=\{1 \ldots k\} \\
k>k_{0}}} \frac{\left|z\left(m_{0}\right)_{r}\right|}{\left|w_{1+r} \cdots w_{l+r-l_{j_{0}, k_{0}, m_{0}}}\right|}< \\
\frac{m_{0}}{m_{0} \cdot l_{k_{0}-1, k_{0}-1, m_{0}}}<\frac{1}{m_{0}} .
\end{gathered}
$$

Hence, it holds 4.4.8.

Evidently $H_{m} \subseteq N\left(y, U_{m}\right)$ by 4 4.4.8), which implies $\overline{B d}\left(N\left(y, U_{m}\right)\right)>0$. Note that $y \in X$ by condition 4.4.3. We conclude that $B_{w}$ is reiteratively hypercyclic on $c_{0}\left(\mathbb{Z}_{+}\right)$.

It is not difficult to see that following the same steps of the proof of the characterization for frequently hypercyclic and $\mathfrak{U}$-frequently hypercyclic weighted shifts on $c_{0}\left(\mathbb{Z}_{+}\right)$given by Bayart and Ruzsa in (7), it is possible to obtain a characterization for reiteratively hypercyclic weigthed shifts on $c_{0}\left(\mathbb{Z}_{+}\right)$. 


\section{DISJOINT HYPERCYCLICITY ALONG FILTERS}

Theorem 96. Let $w=\left(w_{n}\right)_{n \in \mathbb{N}}$ be a bounded sequence of positive integers. Then $B_{w}$ is reiteratively hypercyclic on $c_{0}\left(\mathbb{Z}_{+}\right)$if and only if there exists a sequence $(M(p))_{p \in \mathbb{N}}$ of positive real numbers tending to $+\infty$ and a sequence $(E(p))_{p \in \mathbb{N}}$ of subsets of $\mathbb{N}$ such that

i) for any $p \geq 1, \overline{B d}\left(E_{p}\right)>0$

ii) for any $p, q \geq 1, p \neq q,\left(E_{p}+[0, p]\right) \cap\left(E_{q}+[0, q]\right)=\emptyset$

iii) for any $p \geq 1, \lim _{n \rightarrow \infty, n \in E_{p}+[0, p]} w_{1} \cdots w_{n}=+\infty$

iv) for any $p, q \geq 1$, for any $n \in E_{p}$ and any $m \in E_{q}$ with $m>n$, for any $t \in\{0 \ldots q\}$,

$$
w_{1} \cdots w_{m-n+t} \geq M(p) M(q) .
$$

Remark 97. Now, Theorem 95 tell us that in order to move from syndeticity to reiterative hypercyclicity (for shifts) on $c_{0}\left(\mathbb{Z}_{+}\right)$it suffices to verify condition $i v$ ) "partially" in Theorem 96. A similar statement in the vein of Theorem 95 can be formulated for shifts on $l_{p}\left(\mathbb{Z}_{+}\right)$.

\subsection{Questions}

Concerning the statement of Theorem 91, we were not able to determine whether reiteratively hypercyclicity implies $d$-transitivity or not. On the other hand, in (14) the authors wonder about the existence of a mixing continuous linear operator $T$ such that $\left(T, T^{2}\right)$ is not $d$-transitive. Now, taking into account Corollary 92 , it makes sense to pose the following:

Question 98. Does there exist a reiteratively hypercyclic continuous linear operator $T$ on a separable Banach space such that $\left(T, T^{2}\right)$ is not d-transitive?

On the other hand, in the case the answer to the precedent question is positive, bearing in mind Proposition 83 , it makes sense the following: 
Question 99. Does every reiteratively hypercyclic continuous linear operator $T$ on a separable Banach space is such that $\left(T, \ldots, T^{r}\right)$ satisfies the $d$-FुF Hypercyclicity Criterion for some family $\mathscr{F}$, and any $r \in \mathbb{N}$ ? 


\section{Bibliography}

[1] C. Badea, S. Grivaux, Unimodular eigenvalues, uniformly distributed sequences and linear dynamics. Adv. Math. 211 (2007) 766-793. 15, 39

[2] F. Bayart, S. Grivaux, Frequently hypercyclic operators, Trans. Amer. Math. Soc., 358 (11): 5083-5117, 2006. 10

[3] F. Bayart, S. Grivaux, Invariant Gaussian measures for linear operators on Banach spaces and linear dynamics, Proc. London Math. Soc. (2007) 94 (1): 181-120. 10, 83

[4] F. Bayart, E. Matheron, Dynamics of linear operators, Cambridge Tracts in Mathematics, No. 179, (2009). 2, 9, 10, 16, 47

[5] F. Bayart, E. Matheron, Hypercyclic operators failing the Hypercyclicity criterion on classical Banach spaces J. Funct. Anal., 250 (2) (2007), 426-441. 3

[6] F. Bayart, E. Matheron, (Non)-weakly mixing operators and hypercyclicity sets, Ann. Inst. Four.: 2009. 39 
[7] F. Bayart, I. Ruzsa, Difference sets and frequently hypercyclic weighted shifts, Ergod. Th. \& Dynam. Sys. (2013), available on CJO2013. doi:10.1017/etds.2013.77. 10, 38, 93

[8] V. Bergelson and T. Downarowicz, Large sets of integers and hierarchy of mixing properties of measure-preserving systems, Colloq. Math. 110 (2008), no. 1, 117-150. 25

[9] V. Bergelson and N. Hindman, Partition regular structures contained in large sets are abundant, J. Comb. Theory Ser. A 93 (2001), 18-36. 7. 24, 70, 75

[10] V. Bergelson, R. McCutcheon, Idempotent ultrafilters, multiple weak mixing and Szemerédi's theorem for generalized polynomials, J. Anal. Math. 111 (2010) 77-130. iii, 41, 44, 84

[11] L. Bernal-González, Disjoint hypercyclic operators, Studia Math., 182 (2) (2007), 113-130. 10

[12] J. Bès, Ö. Martin, Compositional disjoint hypercyclicity equals disjoint supercyclicity, Houston J. Math., 38 (4) (2012), 1149-1163. 11

[13] J. Bès, Ö. Martin, A. Peris, Disjoint hypercyclic linear fractional composition operators, J. Math. Anal. Appl., 381 (2011), 843-856. 11

[14] J. Bès, Ö. Martin, A. Peris, S. Shkarin, Disjoint mixing operators, J. Funct. Anal. 263 (2012) 1283-1322. 11, 12, 54, 62, 69, 70, 75, 77, 79. 80, 94

[15] J. Bes, Ö. Martin, R. Sanders, Weighted shifts and disjoint hypercyclicity, to appear in J. Operator Theory. 11, 64, 75 
[16] J. Bes, A. Peris, Disjointness in hypercyclicity, J. Math. Anal. Appl. 336 (2007) 297-315. 10, 11, 12, 49, 62, 63, 76, 77

[17] J. Bes, A. Peris, Hereditarily hypercyclic operators, J. Funct. Anal. 167 (1999), 94-112. 2, 3

[18] G. Costakis, A. Manoussos, I. Parissis, Recurrent linear operators, to appear in Complex Anal. Oper. Theory, arXiv: 1301.1812v2 [math FA] 12 Dec 2013. 42

[19] G. Costakis, I. Parissis, Szemerédi's Theorem, frequent hypercyclicity and multiple recurrence, Math. Scand. 110 (2012) no.2, 251-272. iii, 41, $42,43,48,49,50$

[20] S. M. Duyos-Ruis, Universal functions of the structure of the space of entires functions, Soviet Math. Dokl 30 (1984), no. 3, 713-716. ii

[21] H. Furstenberg, Disjointness in ergodic theory, minimal sets, and a problem in Diophantine approximation, Math. Systems Theory 1 (1967) 1-49. 10

[22] R. M. Gethner, J. H. Shapiro, Universal vectors for operators on spaces of holomorphic functions, Proc. Amer. Math. Soc. 100 (1987) 281-288. ii, 2

[23] S. Grivaux, Hypercyclic operators, mixing operators and the bounded steps problem, J. Operator Theory, 54:1(2005), 147-168. 69

[24] K.-G. Grosse-Erdmann, A. Peris, Linear chaos, Universitext, Springer, London, 2011. 8, 10, 15, 47, 55, 77 
[25] K.-G. Grosse-Erdmann, A. Peris, Weakly mixing operators on topological vector spaces, Rev. Real Acad. Cienc. Exactas Fis. Nat. Ser. A-Mat. 104 (2), 2010, 413-426. 58

[26] N. Hindman, I. Leader and D. Strauss, Infinite partition regular matrices: solutions in central sets, Trans. Amer. Math. Soc. 355 (2003), 1213-1235. 87

[27] N. Hindman, D. Strauss, Algebra in the Stone-Čech compactification. Theory and applications, De Gruyter Expositions in Mathematics 27, (1998). 3, 4, 5, 6, 13, $14,45,86$

[28] N. Hindman, D. Strauss, Density in arbitrary semigroups, Semigr. Forum Vol. 73, (2006), 273-300. 6, 7, 45

[29] C. Kitai, Invariant closed sets for linear operators, Thesis, Univ. of Toronto, 1982. ii, 2

[30] Q. Menet, Existence and non-existence of hypercyclic subspaces, $\mathrm{PhD}$ Thesis, Univ. de Mons, Belgique, 2013. 9

[31] M. di Nasso, Embeddability properties of difference sets, arXiv:1201.5865v1 [math.LO] 27 Jan 2012. 30

[32] S. Rolewicz, On orbits of elements, Studia Math 32 (1969), 17-22. [ii

[33] M. de la Rosa, C. Read, A hypercyclic operator whose direct sum $T \oplus T$ is not hypercyclic, J. Operator Theory, 61 (2) 2009, 369-380. 3

[34] H. N. Salas, Dual disjoint hypercyclic operators, J. Math. Anal. Appl. 374 (1) (2011), 106-117. 11 
[35] H. N. Salas, Hypercyclic weighted shifts, Trans. Amer. Math. Soc. 347 (3) (1995) 993-1004. 68

[36] R. Sanders, S. Shkarin, Existence of disjoint weakly mixing operators that fail to satisfy the Disjoint Hypercyclicity Criterion, J. Math. Anal. Appl., 417 (2) (2014), 834-855.

[37] S. Shkarin, A short proof of existence of disjoint hypercyclic operators, J. Math. Anal. Appl., 367 (2) (2010), 713-715. 11

[38] S. Shkarin, On the spectrum of frequently hypercyclic operators, Proc. Amer. Math. Soc. Volume 137, Number 1, January 2009, 123-134. 10 